\title{
Perturbação dos dados de equações do tipo parabólico em espaços de Banach
}

\author{
Antonio Sergio Munhoz
}

Tese apresentada

ao

Instituto de Matemática e Estatística

da

Universidade de São Paulo

para Obtenção do Grau

de

Doutor em Matemática Aplicada

Área de Concentração: Equações Diferenciais Parciais Orientador(in memorian): Prof. Dr. Daniel Bauman Henry

Orientador: Prof. Dr. Antonio Luiz Pereira

-São Paulo, março de 2003- 


\title{
Perturbação dos dados de equações do tipo parabólico em espaços de Banach
}

\author{
Antonio Sergio Munhoz
}

Este exemplar corresponde à redação final da dissertação corrigida, defendida por Antonio Sergio Munhoz e aprovada pela comissão julgadora.

São Paulo, 9 de abril de 2003.

Banca examinadora:

- Prof. Dr. Antonio Luiz Pereira (orientador) - IME-USP

- Prof. Dr. Luiz Augusto Fernades de Oliveira - IME-USP

- Prof. Dr. Alexandre Nolasco de Carvalho - ICMC-USP

- Prof. Dr. Hildebrando Munhoz Rodrigues - ICMC-USP

- Prof. Dr. Orlando Francisco Lopez - IMECC-UNICAMP 
"Paga mal ao mestre quem se porta sempre como aluno" Assim Falou Zaratustra, Nietzsche

Nunca vi ninguém pagar melhor à criação do que você, meu caro Henry. $E$ é a você que dedico este trabalho.

À Daniel Henry, com grande estima. 


\section{AGRADECIMENTOS}

Às pessoas que estiveram por perto, proporcionando afetividade, ainda que minha atenção seja pouca. Minha mãe Sebastiana, minha mulher Solange, minha filha Camila, minha cunhada Salete e minha sobrinha Daniele. Em particular, à Solange, por me obrigar a aclarar minhas idéias. E também à minha mãe em cujos sonhos tenho um lugar. À Camila cuja ternura me emociona.

Ao Ricardo da Unifei, que perguntava sempre pelo trabalho, obrigando a me autoavaliar. A muitas pessoas que ficavam perguntando quando ia começar o doutorado, como meu sobrinho Ewerson e muitos colegas do IMEUSP, meus agradecimentos.

Ao meu pai Manoel, pelo lirismo que a sua existência me proporcionou.

Aos professores Antonio Luiz Pereira, Luiz Augusto Fernandes de Oliveira e Sérgio Muniz Oliva Filho, que me motivaram a terminar esta trabalho. Em particular, ao Professor Antonio Luiz Pereira, que abraçou a proposta original do professor Henry. Ao professor Luiz Augusto Fernades de Oliveira por possibilitar desenvolver um trabalho muito proveitoso no seu curso da pós em 2002. Em conjunto, a estes dois últimos professores, por terem participado dos seminários de 2002 que foram indispensáveis a esta tese.

Ao professor Marcelo Breda Mourão da Poli-Metal por ter valorizado meu trabalho e pelas colaborações em relação à sua área e pelo atenciosidade com que me trata. Ao professor Alexandre Nolasco de Carvalho do ICMC-USP, pelos poucos, mas estimulantes contatos que tivemos.

Ao meu compadre Sinézio Ferraz Bueno pela companhia agradabilíssima que é e pelos grandes embates que temos travado no http://www.xadrezonline.com.br, do qual tenho saído derrotado na maioria. Mas estou bem neste.

Aos professores, cujos nomes a memória perdeu, mas cujo valor permanece. Em particular, minha professora de matemática da sexta série pela clima excitante com que trabalhava e meu professor particular de matemática e ciências da oitava série por ter proporcionado as tardes que estão entre as melhores da minha vida. À professora Iracema Martin Bund, pela classe que atribuia ao ambiente acadêmico, e por ter me ouvido nos encontros de iniciação científica. Igualmente por este último motivo, à professora Carmen Cardassi e ao professor Hamilton Luiz Guidorizzi. As aulas de graduação com o professor Hamilton também foram cheias de alegria que guardo até hoje, e por isto, meu agradecimento. 
Ao irreproduzível professor Aguinaldo Prandiano Ricieri, professor do ITA e do Anglo, por me influenciar na crença de que há uma luta incrível pela ciência. E também ao professor André Antunha da Poli-Química que também tem este espírito.

Ao meu primo Helinho pelo aventuras criativas com que encheu minhas férias da adolescência.

Aos meus irmãos, pessoas de uma capacidade crítica admirável, o que fortelece nossa inteligência. Em particular, ao Paulo pela atenção e diálogo que nos proporciona.

Aos meus alunos, que, não entende como, manifestam um desagrado instantâneo diante da fraqueza do argumento e não me dão alternativa senão procurar um melhor.

Ao professor Daniel Bauman Henry, pelas demonstrações de balé com que elaborava argumentos difíceis durante as aulas, pela seriedade e beleza de sua redação, e por muitas outras razões. Alguns das suas frases que me ajudaram muito: "não quero pensar sobre isto ", "estou fazendo confusão", "isto é um lixo". Se você quer ter uma idéia da capacidade dele, tenter ler seu livro, sua teoria geométrica ou suas incontáveis notas de aula. . Provavelmente, não há ninguém no mundo capaz de fazê-lo sem sentir um golpe na própria auto-estima, vertigem junto com admiração. Atrás do nome dele pode-se pensar em uma escola, tamanha a extensão e valor de sua produção. 


\section{Resumo}

O objetivo desta tese é fornecer resultados de perturbação para a equação do tipo parabólico em $x, d x / d t+A x=f, t>0, x(0)=\xi$, em um espaço de Banach $X_{0}$ em relação aos dados $\xi, A$ e $f$. O operador $A$ tem domínio constante $X_{1} \subset X_{0}$, densa e continuamente, gera um semigrupo analítico em $X_{0}$ e a sua norma do gráfico é equivalente à norma de $X_{1} ; f$ uma função com valores em $X_{0}$; e $\xi$ um elemento de um espaço intermediário entre $X_{0}$ e $X_{1}$.

\section{Abstract}

The goal of this thesis is to obtain perturbation results for the parabolic type equation in $x, d x / d t+A x=f, t>0, x(0)=\xi$, in a Banach space $X_{0}$ when $\xi, A$ and $f$ change. The operator $A$ has a constant domain $X_{1} \subset X_{0}$, with continuos and dense injection, is setorial in $X_{0}$, and its graph norm is equivalent to the norm of $X_{1}$; the function $f$ has values in $X_{0}$; and $\xi$ is in a intermediate space between $X_{0}$ and $X_{1}$. 


\section{Introdução}

O objetivo deste trabalho é fornecer uma estrutura que permita perturbar os dados de equações do tipo parabólico em espaço de Banach e avaliar o consequente efeito na solução.

Por uma equação do tipo parabólico, entendemos uma equação em $x$, $d x / d t+A x=f, t>0, x(0)=\xi$ em um espaços de Banach $X_{0}$, o operador $A$ com domínio $X_{1} \subset X_{0}$, densa e continuamente, gerador de um semigrupo analítico e com norma do gráfico equivalente à norma de $X_{1}$; a função $f$ com valores em $X_{0}$ e $\xi$ um elemento de um espaço intermediário entre $X_{0}$ e $X_{1}$.

Os dados da equação são $\xi, f$ e $A$. O incomum é ter o próprio operador como um dado.

Ao lado da teoria de perturbação de Kato, o pioneiro no assunto a tratar a questão especificamente para pertubação de dados da equação do tipo parabólico foi Daniel Henry. Nos resultados de perturbação de Henry inclui-se a mudança do operador, mas segundo uma direção bem controlada, como $\mu A$, com variação de $\mu>0$.

A limitação ocorria antes pela escolha tradicional em colocar a solução da equação no domínio da potência fracionária do operador. Além de só em casos bem específicos ser conhecida a dependência deste domínio com o operador, há também dificuldades lógicas.

Por exemplo, o clássico trabalho de Sobolevskii fornece solução para a equação quasilinear $d x / d t+A(t, x) x=f(t, x), t>0, x(0)=\xi$, no espaço $X_{0}$ se $A(0, \xi)$ gera um semigrupo analítico em $X_{0}$, as funções $(t, x) \rightarrow$ $f(t, x), A(t, x)$ regulares com $t \in \mathbb{R}$ e $x \in X^{\alpha}$ onde $X^{\alpha}$ é o domínio da potência $\alpha$-fracionária de $A(0, \xi)$. Mas há adicionalmente a hipótese de que $\xi \in X^{\beta}$, para $\beta>\alpha$. Note a circularidade.

Então para remover esta dificuldade a solução da equação é colocada em um intermediário entre $X_{0}$ e $X_{1}$, mas que não depende de $A$. Obviamente é necessário ver como se modificam as estimativas de Henry envolvendo semigrupos analíticos, $e^{-t A} \xi$, solução de $d x / d t+A x=0, t>0, x(0)=\xi$, e os espaços de potência; e como se modificam as estimativas de Sobolevskii para o operador de evolução $U(t, \tau) \xi$, solução de $d x / d t+A(t) x=0, t>\tau$, também com os espaços de potência.

Entretanto o programa acima ainda não foi feito. As contribuições mais próximas disto estão no livro de Alessandra Lunardi, mas com suas estimativas não é possível repetir muitos resultados de Henry, principalmente os envolvendo comportamento assintótico, ou um substituto para o teorema de existência e unicidade de Sobolevskii mencionado. 
O programa também não foi feito por Amann. Seus resultados são sempre mencionados incluindo vários possíveis escolhas de espaços intermediários, mas a generalidade torna seus teoremas difíceis de usar. Também as estimativas de Sobolevskii não foram repetidas por ele com o mesmo nível de detalhe. Entretanto, com todas as estimativas conseguidas, não foi possível chegar a um teorema para o caso quasilinear equivalente ao [[8], teorema 3.5.2].

A perturbação do operador conforme descrita aqui, com uma topologia que garante que localmente o semigrupo ou operador de evolução estão bem definidos, e demonstração da analiticidade da depência não existem em referência que conheço.

O exemplo de bifurcação de Hopf para sistema de reação-difusão com condições de fronteira não-lineares e operador diferencial linear nas segundas derivadas também constitui referência original. 


\section{Sumário}

1 Espaços Intermediários 11

1.1 Nota Bibliográfica . . . . . . . . . . . . . . . . . . . . 12

1.2 Geração de Espaços Intermediários . . . . . . . . . . . . . . . . 13

1.2.1 Espaços $\left(X_{1}, X_{2}\right)_{\alpha, \infty ; K} \ldots \ldots \ldots \ldots \ldots$

1.2 .2 Espaços $X_{\alpha, 1 ; \infty}(A) \ldots \ldots \ldots \ldots$

1.2 .3 Espaços $X_{\alpha, 1 ; \infty}^{\prime}(A) \ldots \ldots \ldots \ldots \ldots 17$

1.2.4 Espaços $X_{\alpha, 1 ; \infty}^{o}$ e $(X, D(A))_{\alpha, \infty ; K}^{o} \ldots \ldots \ldots . \ldots 28$

1.3 Relação com os espaços de potência fracionária . . . . . . . 33

1.4 Conexão com os resultados de Grisvard . . . . . . . . . . . . . 35

1.5 Estimativas com o resolvente . . . . . . . . . . . 37

2 Estimativas e analiticidade do Semigrupo $\quad 39$

2.1 Estimativas para semigrupo . . . . . . . . . . . . . 40

2.2 Analiticidade do semigrupo . . . . . . . . . . . . 43

3 Estimativas e analiticidade do Operador de Evolução $\quad 51$

3.1 Operador de evolução do tipo parabólico e desigualdade de Gronwall . . . . . . . . . . . . . . . . 52

3.2 Ferramentas do cálculo em espaço de Banach . . . . . . . . . . 56

3.3 Analiticidade do Operador de Evolução . . . . . . . . . . . . 62

3.4 Estimativas para o operador de evolução . . . . . . . . . . 69

4 Elementos da teoria linear $\quad 77$

5 Equação de Evolução Parabólica $\quad 83$

5.1 Topologia . . . . . . . . . . . . . . . . . . . . 84

5.2 Mais cálculo em espaço de Banach . . . . . . . . . . . . . . . 85

5.3 Teorema de existência e unicidade . . . . . . . . . . . . 87 
5.4 Lipschitz continuidade com os dados . . . . . . . . . . . . . 91

5.5 Diferenciabilidade e Analiticidade com os dados . . . . . . . . 92

5.6 Exemplo . . . . . . . . . . . . . . . . . . . 93

5.6 .1 Existência . . . . . . . . . . . . . . . 93

5.6 .2 Bifurcação de Hopf . . . . . . . . . . . . . . . . . 97 


\section{Capítulo 1}

\section{Espaços Intermediários}

É necessário muito pouco para definir espaços intermediários. Dois espaços de Banach continuamente imersos em um espaço de Banach é mais do que o suficiente. Então, supondo este par fixo, se houver mais estrutu$\mathrm{ra}$, as normas podem ter uma forma mais complexa, mas os espaços não se modificam.

Para dois espaços de Banach $X_{0}$ e $X_{1}, X_{1} \subset X_{0}$ partindo do funcional

$$
K(t ; f)=\inf _{f=f_{0}+f_{1}}\left\{\left\|f_{0}\right\|_{0}+t\left\|f_{1}\right\|_{1}\right\},
$$

é possível definir o espaço de Banach $\left(X_{0}, X_{1}\right)_{\alpha, \infty ; K}$ como as funções $f \in$ $X_{0}$ que possuem o valor $\sup \left(t^{-\alpha} K(t ; f)\right)$ finito com sup em $0<t<\infty$ e escolhido como norma do espaço.

Quando existir um operador linear $A$ com domínio $D(A)=X_{1}$ em $X_{0}$, em que $-A$ gera um semigrupo $C_{0}$ em $X_{0}$ então está bem definido o espaço de Banach $X_{\alpha, 1 ; \infty}(A)$ formado pelas funções $f$ com

$$
\|f\|+\sup _{0<s<\infty}\left(s^{-\alpha}\left\|\left(e^{-s A}-I\right) f\right\|\right)
$$

finito e escolhido como norma. Se também o operador $-A$ gera um semigrupo analítico, é possível definir ainda o espaço de Banach $X_{\alpha, 1 ; \infty}^{\prime}(A)$ formado pelas funções $f \in X$ com

$$
\|f\|+\sup _{0<s<\infty}\left(s^{1-\alpha}\left\|A e^{-s A} f\right\|\right)
$$

finito e escolhido como norma. 
Embora as normas possuam formas bem diferentes, valem as igualdades com normas equivalentes

$$
\left(X_{0}, X_{1}\right)_{\alpha, \infty ; K}=X_{\alpha, 1 ; \infty}(A)=X_{\alpha, 1 ; \infty}^{\prime}(A)
$$

para $\alpha \in[0,1]$.

Este último fato é de grande utilidade. Então se $-A_{1}$ e $-A_{2}$ geram semigrupos analíticos em $X_{0}$ com $D\left(A_{1}\right)=D\left(A_{2}\right)$ com normas equivalentes quando $D\left(A_{1}\right)$ e $D\left(A_{2}\right)$ possuem a norma do gráfico então, se $X_{1}=D\left(A_{1}\right)=$ $D\left(A_{2}\right)$, vale $X_{\alpha, 1 ; \infty}^{\prime}\left(A_{1}\right)=X_{\alpha, 1 ; \infty}^{\prime}\left(A_{2}\right)=\left(X_{0}, X_{1}\right)_{\alpha, \infty ; K}$. com normas equivalentes.

Permeia todo este capítulo uma idéia que pode soar estranha. Para definir um operador $A$, é necessário fornecer seu domínio e regra de cálculo. Então necessário fornecer um $X_{1}$ para ser $D(A)$. Depois pedir que $D(A)=X_{1}$ ! Mas uma vez definido o operador ainda que com o domínio $X_{1}$, nada garante que a norma do gráfico de $A$ mantenha-se equivalente à norma de $X_{1}$. E mais se o operador varia com digamos um $\mu$, com domínio fixo $D\left(A(\mu)=X_{1}\right.$ com normas equivalentes para cada $\mu$, nada garante que as constantes de imersão não dependam de $\mu$. Para as aplicações, estas constantes de imersão vão ter seu valor controlado.

Nas aplicações às equações de evolução parabólica, os espaços que são usados são subespaços fechados destes últimos. Os espaços $X_{\alpha, 1 ; \infty}^{o}(A)$ definido como um subespaço de $X_{0}$ cujos elementos obedecem $t^{-\alpha}\left\|\left(e^{-t A}-I\right) f\right\| \rightarrow 0$ se $t \rightarrow 0$ e também os espaços $\left(X_{0}, X_{1}\right)_{\alpha, \infty ; K}^{o}$ que são os elementos de $X_{0}$ que obedecem $t^{-\alpha} K(t ; f) \rightarrow 0$ se $t \rightarrow 0$.

Suponha hipóteses convenientes para existência de potência do operador e semigrupo analítico em $X$. Uma vantagem dos espaços $X_{\alpha, 1 ; \infty}(A)$ sobre os espaços de potência $A^{-\alpha} X=X^{\alpha}(A)$ [[8], página 25] é ser mais estável em relação à perturbação do operador. Então, para dois operadores lineares $A_{1}, A_{2}$, tem-se garantia que $X^{\alpha}\left(A_{1}\right)=X^{\alpha}\left(A_{2}\right)$ se $\left(A_{1}-A_{2}\right) A_{1}^{-\beta} \in L(X)$, algum $\beta \in[0,1)$ ao passo que $X_{\alpha, 1 ; \infty}\left(A_{1}\right)=X_{\alpha, 1 ; \infty}\left(A_{2}\right)$ inclusive se somente $\left(A_{1}-A_{2}\right) A_{1}^{-1}$ é limitado em $X$. Hipóteses mais explítas na observação 1.2.23.

\subsection{Nota Bibliográfica}

Vamos admitir uma parte dos resultados sobre espaços intermediários de Peetre descritos principalmente em [[3], capítulo 3, seções 3.1, 3.2, 3.4 e 3.5], mas sempre com referências explícitas. Nossa guia é incluir tudo que for 
necessário para entender como variam os espaços, variando os operadores. No livro [3], o operador permanece fixo. Além disso, usamos um argumento mais direto que [3] para conseguir constantes mais explícitas nas estimativas.

\subsection{Geração de Espaços Intermediários}

Suponha $X_{1}$ e $X_{2}$ espaços de Banach continuamente imersos em um espaço de Banach $X$. Então os espaços $X_{1} \cap X_{2}$ com norma

$$
\|f\|_{X_{1} \cap X_{2}}=\max \left\{\|f\|_{1},\|f\|_{2}\right\}
$$

e $X_{1}+X_{2}$ com norma

$$
\|f\|_{X_{1}+X_{2}}=\inf _{f=f_{1}+f_{2}}\left(\left\|f_{1}\right\|_{1}+\left\|f_{2}\right\|_{2}\right\}
$$

são espaços de Banach [[3],página 165, proposição 3.2.1].

Definição 1.2.1 $O$ espaço de Banach $Y, Y \subset X$, é um espaço intermediário entre $X_{1}$ e $X_{2}$ se

$$
X_{1} \cap X_{2} \subset Y \subset X_{1}+X_{2}
$$

com imersóes contínuas.

Exemplo 1.2.2 Os próprios espaços $X_{1}$ e $X_{2}$ são espaços intermediários entre $X_{1}$ e $X_{2}$.

Para ver isto, suponhamos $f \in X_{1} \cap X_{2}$. Pela definição de norma em $X_{1} \cap X_{2}$,

então $X_{1} \cap X_{2} \subset X_{1}$.

$$
\|f\|_{1} \leq\|f\|_{X_{1} \cap X_{2}}
$$

Se agora $f \in X_{1}$, como $f=f+0$,

$$
\|f\|_{X_{1}+X_{2}} \leq\|f\|_{1},
$$

e então $X_{1} \subset X_{1}+X_{2}$.

$A$ análise para $X_{2}$ é idêntica.

Exemplo 1.2.3 Vamos descrever os espaços intermediários entre o domínio $D(A)$ de um operador linear $A$ fechado em um espaço de Banach $X$ com $D(A) \subset X$. O espaço $D(A)$ com norma do gráfico, isto é,

$$
\|a\|_{D(A)}=\|a\|+\|A a\|
$$


denotando \|| || como a norma em $X$, é um espaço de Banach graças à hipótese de A ser fechado.

Por um lado

$$
\|f\|_{D(A) \cap X}=\|f\|_{D(A)}
$$

e $D(A) \cap X=D(A)$ com normas iguais.

E por outro lado

$$
\|f\|_{D(A)+X}=i n f_{a \in D(A)}(\|f-a\|+\|a\|+\|A a\|) \geq\|f\|,
$$

e, com o exemplo anterior, $\|f\|_{D(A)+X}=\|f\|$. Então $D(A)+X=X$ com normas iguais.

Portanto os espaços intermediários entre $D(A)$ e $X$ são os espaços de Banach $Y$ com $D(A) \subset Y \subset X$ com imersões contínuas.

Exemplo 1.2.4 Suponha o operador linear $A, A: D(A) \subset X \rightarrow X$, setorial $e \operatorname{Re}(\sigma(A))>0$. Sabemos que a potência $A^{\alpha}, \alpha \in[0,1]$, está bem definida e seu domínio com norma $\left\|A^{\alpha} y\right\|, y \in D\left(A^{\alpha}\right)$, é um espaço de Banach $e$ obedece às imersões contínuas

$$
D(A) \subset D\left(A^{\alpha}\right) \subset X,
$$

[[8], seção 1.4, páginas 24 e 25].

Então, pelo exemplo anterior, $D\left(A^{\alpha}\right)$ é um espaço intermediário entre $D(A)$ e $X$.

\subsubsection{Espaços $\left(X_{1}, X_{2}\right)_{\alpha, \infty ; K}$}

Existem diversos métodos para gerar espaços intermediários. Partindo de um par de espaços de Banach, Butzer e Berens [[3], seção 3.2] explicam os chamados métodos $K$ e $J$. Embora sejam equivalentes [[3], seção 3.2.3, teorema 3.2.12], escolhemos o método $K$ por ser operacionalmente o mais simples dos dois.

Definição 1.2.5 Sejam $X_{1}$ e $X_{2}$ espaços de Banach continuamente imersos em um espaço de Banach $X$. Seja

$K\left(t, f, X_{1}, X_{2}\right)=K(t, f):=\inf _{f=f_{1}+f_{2}}\left(\left\|f_{1}\right\|_{1}+t\left\|f_{2}\right\|_{2}\right), f \in X_{1}+X_{2}, 0<t<\infty$. 
Para $\alpha \in[0,1]$, chamamos de $\left(X_{1}, X_{2}\right)_{\alpha, \infty ; K}$ o espaço formados pelas funções $f \in X_{1}+X_{2}$ com $\sup _{0<t<\infty}\left(t^{-\alpha} K(t ; f)\right)$ finito. Neste caso, a norma no espaço será

$$
\|f\|_{\alpha, \infty ; K}:=\sup _{0<t<\infty}\left(t^{-\alpha} K(t ; f)\right) .
$$

Observação 1.2.6 Sabemos que estes espaços são mesmo espaços intermediários conforme [Butzer e Berens[3], página 168, proposição 3.2.4].

Observação 1.2.7 Estamos particularmente interessados nos espaços intermediários gerados pelo método $K$ quando $X_{1}=X$ e $X_{2}=D(A)$ para $A$ operado linear fechado em $X$.

Definição 1.2.8 Para dois espaços de Banach $Z$ e $W$, a igualdade $Z \doteq W$ signica $Z=W$ com normas equivalentes.

Proposição 1.2.9 Suponha A, B operadores lineares fechados em um espaço de Banach $X$. Então

1. Se $D(A)=D(B)$ e $D(A) \subset D(B)$ com $\|f\|_{D(B)} \leq c_{1}\|f\|_{D(A)}$ para todo $f \in D(A)$ então

$$
\begin{gathered}
(X, D(A))_{\alpha, \infty ; K} \subset(X, D(B))_{\alpha, \infty ; K}, \\
\text { e se } f \in(X, D(A))_{\alpha, \infty ; K} \\
\|f\|_{(X, D(B))_{\alpha, \infty ; K}} \leq \max \left\{1, c_{1}\right\}\|f\|_{(X, D(A))_{\alpha, \infty ; K}}
\end{gathered}
$$

2. Se $D(A) \doteq D(B)$,

$$
(X, D(A))_{\alpha, \infty ; K} \doteq(X, D(B))_{\alpha, \infty ; K} .
$$

Prova. Denotemos $D(A)=D_{A}$ e $D(B)=D_{B}$ e $\left(X, D_{A}\right)_{\alpha, \infty ; K}=$ $D_{A}(\alpha, \infty),\left(X, D_{B}\right)_{\alpha, \infty ; K}=D_{B}(\alpha, \infty)$. Suponha $f \in D_{A}(\alpha, \infty)$. Por hipótese, existe uma constante $c_{1}$ não dependente de $a \in D_{A}$ para $\|a\|_{D_{B}} \leq c_{1}\|a\|_{D_{A}}$. Então, para $x \in X, t \in(0, \infty)$,

$$
t^{-\alpha} \inf _{f=x+a}\left(\|x\|+t\|a\|_{D_{B}}\right) \leq t^{-\alpha} \inf _{f=x+a}\left(\|x\|+t c_{1}\|a\|_{D_{A}}\right) .
$$

Portanto $\|f\|_{D_{B}(\alpha, \infty)} \leq \max \left\{1, c_{1}\right\}\|f\|_{D_{A}(\alpha, \infty)}$ e $D_{A}(\alpha, \infty) \subset D_{B}(\alpha, \infty)$, provando 1 .

A prova da parte 2 segue diretamente de 1 , uma vez que $D(A) \doteq D(B)$ equivale a $D(A)=D(B), D(A) \subset D(B)$ e $D(B) \subset D(A)$. 
Proposição 1.2.10 Suponha que exista no conjunto de operadores com domínio constante $\mathcal{A} \subset\left\{A: X_{1} \subset X \rightarrow X\right.$, A linear fechado em $\left.X\right\}$ um operador $A_{0} \operatorname{com} D\left(A_{0}\right) \doteq D(A)$ e imersões não dependentes de $A \in \mathcal{A}$, isto é, existem constantes $c_{1}$ e $c_{2}$ para $\|f\|_{D\left(A_{0}\right)} \leq c_{1}\|f\|_{D(A)}$ e $\|f\|_{D(A)} \leq c_{2}\|f\|_{D\left(A_{0}\right)}$ para todo $f \in D\left(A_{0}\right)=D(A)$ e todo $A \in \mathcal{A}$. Então

1.Se $A \in \mathcal{A}$

$$
\begin{gathered}
(X, D(A))_{\alpha, \infty ; K} \doteq\left(X, D\left(A_{0}\right)\right)_{\alpha, \infty ; K} \\
\text { ese } f \in(X, D(A))_{\alpha, \infty ; K}=\left(X, D\left(A_{0}\right)\right)_{\alpha, \infty ; K} \\
\|f\|_{(X, D(A))_{\alpha, \infty ; K}} \leq \max \left\{1, c_{1}\right\}\|f\|_{\left(X, D\left(A_{0}\right)\right)_{\alpha, \infty ; K}}, \\
\|f\|_{\left(X, D\left(A_{0}\right)\right)_{\alpha, \infty ; K}} \leq \max \left\{1, c_{2}\right\}\|f\|_{(X, D(A))_{\alpha, \infty ; K}} .
\end{gathered}
$$

2. Se $A, B \in \mathcal{A}$

$$
\begin{gathered}
(X, D(A))_{\alpha, \infty ; K} \doteq(X, D(B))_{\alpha, \infty ; K}, \\
\text { e se } f \in(X, D(A))_{\alpha, \infty ; K}=(X, D(B))_{\alpha, \infty ; K} \\
\|f\|_{(X, D(A))_{\alpha, \infty ; K}} \leq \max \left\{1, c_{1} c_{2}\right\}\|f\|_{(X, D(B))_{\alpha, \infty ; K}}, \\
\|f\|_{(X, D(B))_{\alpha, \infty ; K}} \leq \max \left\{1, c_{1} c_{2}\right\}\|f\|_{(X, D(A))_{\alpha, \infty ; K}} .
\end{gathered}
$$

3. Se $A \in \mathcal{A}$ e $X_{1} \doteq D\left(A_{0}\right)$ então, uniformemente em $\mathcal{A}$,

$$
(X, D(A))_{\alpha, \infty ; K} \doteq\left(X, X_{1}\right)_{\alpha, \infty ; K}
$$

Prova. A prova de 1 segue diretamente da parte 1 de 1.2 .9 assim como a prova da parte 2 desde que $D(A) \subset D(B)$ com $\|f\|_{D(A)} \leq c_{1} c_{2}\|f\|_{D(B)}$ se $f \in D(A)=D(B), A, B \in \mathcal{A}$.

\subsubsection{Espaços $X_{\alpha, 1 ; \infty}(A)$}

Embora estes espaços não sejam extensivamente usados neste trabalho, é conveniente defini-los por pedirem menos do operador $A$ do que os espaços $X_{\alpha, 1 ; \infty}^{\prime}(A)$ definidos adiante e para não perder o roteiro seguido por [[3], capítulo 3].

Suponha que o operador $-A, A: D(A) \subset X \rightarrow X$, gera um semigrupo $C_{0}$ unifomemente limitado em $X$. 
O espaço $X_{\alpha, 1 ; \infty}(A)$ é formado pelas $f \in X$ que tem

$$
\sup _{0<s<\infty}\left(s^{-\alpha}\left\|\left(e^{-s A}-I\right) f\right\|\right)
$$

finito. Este espaço com norma

$$
\|f\|_{\alpha, 1 ; \infty}=\|f\|+\sup _{0<s<\infty}\left(s^{-\alpha}\left\|\left(e^{-s A}-I\right) f\right\|\right)
$$

é um espaço de Banach intermediário entre $D(A)$ e $X$ se $\alpha \in(0,1]$ conforme [[3], página 160, proposição 3.1.3].

Analogamente, definimos os espaços $X_{\alpha, r ; \infty}(A), r \geq 1$, inteiro com norma

$$
\|f\|_{\alpha, r ; \infty}=\|f\|+\sup _{0<s<\infty}\left(s^{-\alpha}\left\|\left(e^{-s A}-I\right)^{r} f\right\|\right)
$$

que também são espaços intermediários entre $D\left(A^{r}\right)$ e $X$ para $\alpha \in(0, r]$ novamente por [[3], página 160, proposição 3.1 .3$]$.

\subsubsection{Espaços $X_{\alpha, 1 ; \infty}^{\prime}(A)$}

Definição 1.2.11 Seja $A: D(A) \subset X \rightarrow X$, um operador linear e suponha $q u e-A$ é gerador de um semigrup analítico $e^{-s A}$ em $X$. Dizemos que $f \in$ $X_{\alpha, 1 ; \infty}^{\prime}(A)$ se $f \in X$ e $\sup _{0<s<\infty}\left(s^{1-\alpha}\left\|\left(A e^{-s A} f\right)\right\|\right)$ é finito. Sua norma é

$$
\|f\|_{\alpha, 1 ; \infty}^{\prime}=\|f\|+\sup _{0<s<\infty}\left(s^{1-\alpha}\left\|\left(A e^{-s A} f\right)\right\|\right) .
$$

Analogamente para $r \geq 1$, definimos $X_{\alpha, r ; \infty}^{\prime}(A)$ com norma

$$
\|f\|_{\alpha, r ; \infty}^{\prime}=\|f\|+\sup _{0<s<\infty}\left(s^{r-\alpha}\left\|\left(A e^{-s A} f\right)\right\|\right) .
$$

Observação 1.2.12 Os espaços $X_{\alpha, r ; \infty}(A), \alpha \in(0, r]$ são espaços intermediários entre $D\left(A^{r}\right)$ e $X$ [[3], página 207, proposição 3.5.2].

Definição 1.2.13 Sejam $X_{0}$ e $X_{1}$ espaços de Banach com $X_{1} \subset X_{0}$ contínua e densamente. Seja

$$
\begin{aligned}
\mathcal{H}\left(X_{1}, X_{0}\right)= & \left\{A, A: X_{1} \rightarrow X_{0}, \text { linear }, D(A) \doteq X_{1},\right. \text { isto é, } \\
& \exists \bar{C}_{A}>0, \overline{\bar{C}}_{A}>0 \text { para } D(A) \subset X_{1} \text { e } X_{1} \subset D(A) \\
& \text { com constantes de imersão } \bar{C}_{A} \text { e } \overline{\bar{C}}_{A} \\
& \{\mathbb{R e} \lambda \geq 0\} \subset \rho(-A) \text { e } \exists C_{A}>0 \text { tal que } \\
& \left.\left\|(\lambda+A)^{-1}\right\|_{L\left(X_{0}\right)} \leq \frac{C_{A}}{|\lambda|+1} \text { se } \mathbb{R e} \lambda \geq 0\right\}
\end{aligned}
$$


Um conjunto $\mathcal{A} \subset \mathcal{H}\left(X_{1}, X_{0}\right)$ é regular se existe uma constante $C_{\mathcal{A}}$ tal que $\bar{C}_{A} \leq C_{\mathcal{A}}, \overline{\bar{C}}_{A} \leq C_{\mathcal{A}}, C_{A} \leq C_{\mathcal{A}}$ para todo A pertencente a $\mathcal{A}$.

Observação: com freqüência, referência a esta definição implica referência à proposição 1.2.19.

Observação 1.2.14 Note que se $\mathcal{A} \in \mathcal{H}\left(X_{1}, X_{0}\right)$ é um conjunto regular então $\|x\|_{D(A)} \leq\left(C_{\mathcal{A}}\right)^{2}\|x\|_{D(B)}$ para todo $A, B \in \mathcal{A}$.

Observação 1.2.15 Foram retiradas da definição informações escritas nas entrelinhas. Então, embora não esteja escrito, como, digamos, $0 \in \rho(A)$, necessariamente $A$ é um operador fechado, pois todo operador com resolvente não vazio é fechado.

Observação 1.2.16 A redação da definição tem pontos em comum com a a definição de [Sobolevskii[1], página 10, seção 2]. Uma das diferenças é que lá o conjunto os operadore são dependentes de $t, A(t)$. Mas enquanto estivermos pensando estritamente na descrição dos espaços e na estimativas básicas envolvendo semigrupos, assunto do próximo capítulo, a restrição a este tipo de conjunto é uma restrição desnecessária que obscurece a análise.

Observação 1.2.17 Talvez seja útil pensar num exemplo bem simples. Suponha $X_{0}$ e $X_{1}$ o conjunto dos números complexos. Então um operador linear em $X_{0}$ pode ser pensado como um número $A$, tendo no domínio qualquer número complexo. Suponha, digamos, $A \geq 1$ e $A \in \mathbb{R}$, então se $\lambda=x+i y,|\lambda+A|^{2}=|\lambda|^{2}+2 x A+A^{2} \geq|\lambda|^{2}+1$ sempre que $x \geq 0$. Então $|\lambda+A|^{-1} \leq 1 / \sqrt{|\lambda|^{2}+1}$. Uma vez que a função $f(x)=(x+1) / \sqrt{x^{2}+1}$ possui $2 / \sqrt{2}$ como máximo, então $|\lambda+A|^{-1} \leq(2 / \sqrt{2}) /(|\lambda|+1)$. Seja $\mathcal{A}=\{A \in \mathbb{R}, 1 \leq A \leq 100\}$. Se $N \in \mathcal{A},\|z\|_{D(N)}=(N+1)|z|$. Portanto $\|z\|_{D(N)} \leq 101|z| e|z| \leq 1 / 2\|z\|_{D(N)}$. Uma escolha possível para $C_{\mathcal{A}}$ é $\max \{2 / \sqrt{2}, 101,1 / 2\}=101$.

Observação 1.2.18 Quando os operadores $A$ variam em um conjunto regular $\mathcal{A} \subset \mathcal{H}\left(X_{1}, X_{0}\right)$ como aqui, como demonstrada a seguir, os espaços $X_{\alpha, 1 ; \infty}^{\prime}(A)$ não só não se modificam como também fornecem normas equivalentes. 
Proposição 1.2.19 Se $A \in \mathcal{A}$ então $A$ gera um semigrupo analítico $e^{-t A}$ $t \geq 0$ e existem constantes $c_{0}$ e $c_{1}$ e $\delta>0$ para

1. $\left\|e^{-t A}\right\| \leq c_{0} e^{-\delta t}$,

2. $\left\|A e^{-t A}\right\| \leq c_{1} e^{-\delta t} t^{-1}$,

uniformemente em $A \in \mathcal{A}$ com normas em $L(X)$.

Prova. É possível perturbar o conjunto resolvente de $A, \rho(A)$ para conter não só $\mathbb{R} e \lambda \leq 0$ mas também $0 \cup\{\phi \leq \arg (\lambda) \leq \pi\}$, algum $\phi \in(0, \pi / 2)$, obedecendo a desigualdade em 1 em 1.2.13 [Hoppenstadt's[7], apêndice]. Então $A$ é setorial, e, portanto, gera um semigrupo analítico $e^{-t A}[$ Henry[8], página 20, teorema 1.3.4] ou [Pazy[11], página 61, teorema 5.2]. Note também que $0 \in \rho(A)$, então se $|\lambda|\left\|A^{-1}\right\|<1, \lambda \in \rho(A)$ [Butzer e Berens[3], página 31, proposição 1.3.2]. Portanto o resolvente $\rho(A)$ contém também a bola de centro 0 e raio $1 / C_{\mathcal{A}}$ e então podemos tomar $\delta=1 / C_{\mathcal{A}} \cos (\phi)$. O fato de que $c_{0}$, $c_{1}$ não dependem de $A \in \mathcal{A}$ vem do fato de que $(|\lambda|+1)\left\|(\lambda+A)^{-1}\right\|_{L(X)} \leq C_{\mathcal{A}}$ uniformemente em $\mathcal{A}$ e em $\{\mathbb{R} e \lambda \geq 0\}$.

Teorema 1.2.20 Seja $\mathcal{A}$ como na definição 1.2.13. Então, para $\alpha \in(0,1]$,

1. $(X, D(A))_{\alpha, \infty ; K} \doteq X_{\alpha, 1 ; \infty}^{\prime}(A)$ com constantes de imersões não dependentes de $A \in \mathcal{A}$.

2. $X_{\alpha, 1 ; \infty}^{\prime}(A) \doteq X_{\alpha, 1 ; \infty}^{\prime}(B)$, com constantes de imersões não dependentes de $A, B \in \mathcal{A}$.

3. $\left(X, X_{1}\right)_{\alpha, \infty ; K} \doteq X_{\alpha, 1 ; \infty}^{\prime}(A)$ com constantes de imersões não dependentes de $A \in \mathcal{A}$.

Prova. Para $A \in \mathcal{A}$, suponha $f \in X_{\alpha, 1 ; \infty}^{\prime}(A)$. Podemos decompor $f=$ $f-e^{-s A} f+e^{-s A} f$. Temos $\left(f-e^{-s A} f\right) \in X$ e, quando $s>0, e^{-s A} f \in D(A)$. Portanto, denotando a um elemento de $D(A)$ e $x$ um elemento de $X$, para $s>0$,

$$
\begin{aligned}
& \inf _{f=x+a}(\|x\|+s\|a\|+s\|A a\|) \\
& \quad \leq\left\|\left(I-e^{-s A}\right) f\right\|+s\left\|e^{-s A} f\right\|+s\left\|A e^{-s A} f\right\| .
\end{aligned}
$$


Com a estimativa de 1.2 .25 e 1.2 .26

$$
\begin{aligned}
& \|f\|_{\alpha, \infty ; K} \\
& \quad \leq \frac{1}{\alpha} \sup _{0<s<\infty}\left(s^{1-\alpha}\left\|A e^{-s A} f\right\|\right) \\
& \quad+\sup _{0<s<\infty}\left(c_{0} e^{-\delta s} s^{1-\alpha}\|f\|+s^{1-\alpha}\left\|A e^{-s A} f\right\|\right)
\end{aligned}
$$

Como $\max _{0<s<\infty}\left\{s^{1-\alpha} e^{-\delta s}\right\}=\delta^{\alpha-1} / e$,

$$
\begin{aligned}
& \|f\|_{\alpha, \infty ; K} \\
& \quad \leq \max \left\{1+1 / \alpha, c_{0} \delta^{\alpha-1} / e\right\}\|f\|_{\alpha, 1 ; \infty}^{\prime}
\end{aligned}
$$

e então $f \in(X, D(A))_{\alpha, \infty ; K}$.

Inversamente, suponha $f \in X, D(A))_{\alpha, \infty ; K}$. Inicialmente, note que $\|f\| \leq$ $\|f\|_{\alpha, \infty ; K}$, pois para $x \in X$ e $a \in D(A) \operatorname{com} f=x+a$,

$$
\|x\|+s\|a\|+s\|A a\| \geq\|f\|+(s-1)\|a\|+s\|A a\| .
$$

Portanto, se $s \geq 1$,

$$
s^{-\alpha} \inf _{f=x+a}(\|x\|+s\|a\|+s\|A a\|) \geq s^{-\alpha}\|f\| .
$$

Mas $\sup _{s \geq 1}\left(s^{-\alpha}\|f\|\right)=\|f\|$ e, então,

$$
\sup _{s \geq 1}\left(s^{-\alpha} \inf _{f=x+a}(\|x\|+s\|a\|+s\|A a\|)\right) \geq\|f\|,
$$

implicando, como desejado, $\|f\| \leq\|f\|_{\alpha, \infty ; K}$.

Portanto, a parcela problemática para avaliar a norma de $f$ em $X_{\alpha, 1 ; \infty}(A)$ é $\sup _{0<s<\infty}\left(s^{1-\alpha}\left\|A e^{-s A} f\right\|\right)$.

Pelo lema 1.2.22,

$$
s\left\|A e^{-s A} f\right\| \leq \max \left\{c_{0}, c_{1}\right\} K(s, f, X, D(A)) .
$$

Multiplicando por $s^{-\alpha}$, tomando o sup à direita e em seguida à esquerda quando $s \in(0, \infty)$,

$$
\begin{aligned}
& \left.\sup _{0<s<\infty}\left(s^{1-\alpha}\left\|A e^{-s A} f\right\|\right)\right) \\
& \leq \max \left\{c_{0}, c_{1}\right\} \sup _{0<s<\infty}\left(\left(s^{-\alpha} K(s, f)\right)\right) .
\end{aligned}
$$


Portanto

$$
\|f\|_{\alpha, 1 ; \infty} \leq\left(1+\max \left\{c_{0}, c_{1}\right\}\right)\|f\|_{\alpha, 1 ; K}
$$

concluindo a prova da parte 1 .

Esta parte juntamente com a proposição 1.2 .10 e a definição de $\mathcal{A}$ provam a parte 2 .

Observação 1.2.21 Alessandra Lunardi[16] usa como norma para $X_{\alpha, 1 ; \infty}^{\prime}(A)$ somente $\sup _{0<s<\infty}\left(s^{1-\alpha}\left\|A e^{-s A} f\right\|\right)$. Entretanto a equivalência está mantida desde que $f=-\lim _{\epsilon \rightarrow 0} \int_{\epsilon}^{N} A e^{-s A} f d s-e^{-N A} f$ e $e^{-N A} f \rightarrow 0$ fazendo $N \rightarrow \infty$.

Lema 1.2.22 Suponha $A \in \mathcal{A}, \mathcal{A}$ como em 1.2.13. Então para todo $s>0 e$ $f \in X_{0}$

$$
s\left\|A e^{-s A} f\right\| \leq \max \left\{c_{0}, c_{1}\right\} K(s, f, X, D(A)) .
$$

Prova. Suponha $f=x+a, x \in X$ e $a \in D(A)$. Então

$$
\left\|A e^{-s A} f\right\| \leq c_{1} e^{-\delta s} s^{-1}\|x\|+c_{0} e^{-\delta s}\|A a\|
$$

Resulta

$$
\begin{aligned}
& s\left\|A e^{-s A} f\right\| \\
& \quad \leq \max \left\{c_{0}, c_{1}\right\}((\|x\|+s\|a\|+s\|A a\|)) .
\end{aligned}
$$

Como a desigualdade vale para todo $x \in X, a \in D(A) \operatorname{com} f=x+a$,

$$
\left.\left.s\left\|A e^{-s A} f\right\|\right) \leq \max \left\{c_{0}, c_{1}\right\} \inf _{f=x+a}(\|x\|+s\|a\|+s\|A a\|)\right),
$$

concluindo a prova.

Observação 1.2.23 Sejam $X_{0}$ e $X_{1}$ espaços de Banach com $X_{1} \subset X_{0}$ densa e continuamente. Sejam $A_{1}, A_{2}: X_{1} \rightarrow X_{0}$ operadores lineares que obedecem $D\left(A_{1}\right) \doteq X_{1}, A_{1} A_{2}^{-1}, A_{2} A_{1}^{-1} \in L\left(X_{0}\right)$ e $(|\lambda|+1)\left\|\left(\lambda+A_{i}\right)^{-1}\right\|_{L\left(X_{0}\right)} \leq C<\infty$ quando $\lambda \in\{\mathbb{R e} \lambda \geq 0\}$ para $i=1,2$. Então $\mathcal{A}=\left\{A_{1}, A_{2}\right\}$ é um conjunto regular de $\mathcal{H}\left(X_{1}, X_{0}\right)$. Obviamente $\left(A_{1}-A_{2}\right) A_{1}^{-1}$ é limitado em $X$, mas isto não é garantia para igualdade entre os espaços de potência $X^{\alpha}\left(A_{1}\right)$ e $X^{\alpha}\left(A_{2}\right)$, pois o resultado de perturbação conhecido [Henry[8], página 28, teorema 1.4.6] pede $\left(A_{1}-A_{2}\right) A_{1}^{-\beta}$ limitado em $X_{0}$, algum $\beta \in[0,1)$. Exceto em casos bem particulares, como, por exemplo, $A_{1}-A_{2} \in L\left(X_{0}\right)$, não é fácil verificar esta condição. Mas a restrição $A_{1} A_{2}^{-1}, A_{2} A_{1}^{-1} \in L\left(X_{0}\right)$ é prontamente comprovável em muitos casos. Há, portanto, maior liberdade na 
variação do operador sem mudança dos espaços o que justifica a substituição dos espaços de Potência com o quais o professor Henry conseguiu muito em equações semilineares sem variação do operador, exceto com a perturbação mencionada, pelos espaços intermediários de Peetre se permitirmos equações com variação do operador.

Exemplo 1.2.24 Por exemplo, se $S=-\Delta+c, D(S)=\left\{f \in W^{2, p}(\Omega)\right.$, $a(x) \partial f / \partial N+b(x) f=0\}, \partial / \partial N$ é a derivada normal exterir a um aberto limitado regular de $R^{n}, \Omega$ e a,b funçôes $C^{1}(\bar{\Omega})$. Suponha $A=-q(x) \Delta+c, q \in$ $C(\bar{\Omega}), q_{1} \geq q(x) \geq q_{0}>0, D(A)=\left\{f \in W^{2, p}(\Omega), a(x) \partial f / \partial N+b(x) f=0\right\}$. A e $S$ são fechados como operadores lineares em $X_{0}=L_{p}(\Omega)$ se $2-n / p>1$, caso em que $W^{2, p} \subset C^{1}$.

Há duas abordagens possiveis. Definindo $X_{1}=\left\{f \in W^{2, p}(\Omega), a(x) \partial f / \partial N+\right.$ $b(x) f=0\}$ com a norma de $W^{2, p}$ e então demonstrando que $X_{1} \doteq D(S)$ e $X_{1} \doteq D(A)$, com as estimativas para operadores elípticos. A outra abordagem mais direta é definir $X_{1}=D(S)$ com a norma do gráfico de $S$. Seja \| \| a norma em $X_{0}$. Seja $f \in X_{1}$. Por um lado $\|(-\Delta+c) f\| \leq$ $\|-(p(x) \Delta+c) f\|+\left(p_{1}+1\right)\|f\|$. E, por outro lado, $\|(-p(x) \Delta+c) f\| \leq$ $p_{1}\|(-\Delta+c)\|+p_{1} c\|f\| / p_{0}$. Então $D(A) \doteq X_{1}$.

Supondo $c>0$ grande o suficiente e também que $b(x) \geq 0$, então $S, A \in$ $\mathcal{H}\left(X_{1}, X_{0}\right)$; por exemplo, veja [[1], página 54]. Neste caso, também é verdade que $X_{\alpha, 1 ; \infty}^{\prime}(S) \doteq X_{\alpha, 1 ; \infty}^{\prime}(A)$.

Lema 1.2.25 Suponha $A \in \mathcal{A}, \mathcal{A}$ como em 1.2.13. Então se $x \in X_{\alpha, 1 ; \infty}^{\prime}(A)$, $\alpha \in(0,1]$,

$$
\left\|\left(e^{-t A}-I\right) x\right\| \leq \frac{t^{\alpha}}{\alpha}\|x\|_{\alpha, 1 ; \infty}^{\prime},
$$

em que \|\|$_{\alpha, 1 ; \infty}^{\prime}$ é a norma em $X_{\alpha, 1 ; \infty}^{\prime}(A)$. E se $\alpha=0$,

$$
\left\|\left(e^{-t A}-I\right) x\right\| \leq\left(c_{0}+1\right)\|x\|_{0,1 ; \infty}^{\prime},
$$

Prova. Conforme [Butzer e Berens[3], relação 2.3.1, página 111]

$$
\left(e^{-t A}-I\right) x=-\lim _{\epsilon \rightarrow 0} \int_{\epsilon}^{t} A e^{-s A} x d s,
$$

válida mesmo que se $x \in X$ somente.

Como $x \in X_{\alpha, 1 ; \infty}^{\prime}(A)$ e, portanto, $\sup _{0<s<\infty}\left\|s^{1-\alpha} A e^{-s A} x\right\|<\infty$ justificando a estimativa

$$
\left\|\int_{\epsilon}^{t} A e^{-s A} x d s\right\| \leq \frac{t^{\alpha}-\epsilon^{\alpha}}{\alpha} \sup _{0<s<\infty}\left\|s^{1-\alpha} A e^{-s A} x\right\| .
$$


Tomando o limite para $\epsilon \rightarrow 0 \mathrm{em}$

$$
\left\|\left(e^{-t A}-I\right) x\right\| \leq \frac{t^{\alpha}-\epsilon^{\alpha}}{\alpha} \sup _{0<s<\infty}\left\|s^{1-\alpha} A e^{-s A} x\right\|,
$$

concluímos a prova.

Observação 1.2.26 Note que a prova fornece uma estimativa melhor, fornecendo como majorante

$$
\frac{t^{\alpha}}{\alpha} \sup _{0<s<\infty}\left\|s^{1-\alpha} A e^{-s A} x\right\|
$$

em vez de

$$
\frac{t^{\alpha}}{\alpha}\left(\|x\|+\sup _{0<s<\infty}\left\|s^{1-\alpha} A e^{-s A} x\right\|\right) .
$$

Proposição 1.2.27 Suponha $A \in \mathcal{A}, \mathcal{A}$ como em 1.2.13. Então, sempre com constantes de imersão não dependentes de $A \in \mathcal{A}$,

1. $(X, D(A))_{0, \infty ; K} \doteq X$ com normas iguais.

2. Se $\alpha \leq 0, X_{\alpha, 1 ; \infty}^{\prime}(A) \doteq X$, com constantes de imersões não dependentes de $A \in\{\mathcal{A}\}$.

3. $(X, D(A))_{0, \infty ; K} \doteq X_{0,1 ; \infty}^{\prime}(A)$, com constandes de imersões não dependentes de $A \in\{\mathcal{A}\}$.

4. $\left(X, X_{1}\right)_{0, \infty ; K} \doteq X$, com constandes de imersões não dependentes de $A \in\{\mathcal{A}\}$.

Prova. Para provar 1, a dificuldade é provar a imersão $X \subset(X, D(A))_{0, \infty ; K}$, pois a imersão inversa segue diretamente da definição da norma em $(X, D(A))_{0, \infty ; K}$

$$
\|f\|_{0, \infty ; K}=\sup _{0<s<\infty} \inf _{f=x+a}(\|x\|+s\|a\|+s\|A a\|)
$$

onde, como sempre, $x \in X$ e $a \in D(A)$, e do fato $f=f+0$ que implica $\|f\|_{0, \infty ; K} \leq\|f\|$.

Para prova da inversa, suponha $s \geq 1$. Neste caso

$$
\inf _{f=x+a}(\|x\|+s\|a\|+s\|A a\|) \geq \inf _{a}(\|f\|+(s-1)\|a\|+\|A a\|) \geq\|f\| .
$$


Mas ampliar o intervalo só aumenta o sup, portanto

$$
\|f\|_{0, \infty ; K} \geq\|f\|
$$

concluindo a prova de 1 .

Pela definição de norma em $X_{\alpha, 1 ; \infty}^{\prime}(A)$,

$$
\|f\|_{\alpha, 1 ; \infty}=\|f\|+\sup _{0<s<\infty}\left(s^{1-\alpha}\left\|A e^{-s A} f\right\|\right) \text {, }
$$

a imersão $X_{\alpha, 1 ; \infty}^{\prime}(A) \subset X$ é óbvia.

Inversamente suponha $f \in X$. Pela proposição 1.2.19,

$$
\sup _{0<s<\infty}\left(s^{1-\alpha}\left\|A e^{-s A} f\right\|\right) \leq c_{1}\|f\| \sup _{0<s<\infty}\left(s^{-\alpha} e^{-\delta s}\right),
$$

e como $\max _{0<s<\infty}\left(s^{-\alpha} e^{-\delta s}\right)=(-\alpha / \delta)^{-\alpha} e^{\alpha}$ segue

$$
\|f\|_{\alpha, 1 ; \infty} \leq\left(1+c_{1}(-\alpha / \delta)^{-\alpha} e^{\alpha}\right)\|f\|,
$$

completando a prova de 2 .

A parte 3 segue diretamente de 1 e 2; explicitamete $\|f\|_{0,1 ; \infty} \leq\left(1+c_{1}\right)\|f\|_{0, \infty ; K}$ e $\|f\|_{0, \infty ; K} \leq\|f\|_{0,1 ; \infty}$.

Teorema 1.2.28 Suponha $A \in \mathcal{A}, \mathcal{A}$ como em 1.2.13. Seja $Y$ um espaço de Banach e $Y \subset X$ através de imersão contínua. Suponha ainda que se $x \in D(A)$ então $x \in Y$ e também

$$
\|x\|_{Y} \leq C\|A x\|^{\alpha}\|x\|^{1-\alpha}
$$

para algum $\alpha, \alpha \in[0,1)$ e uma constante $C>0$. Ou equivalentemente existe $K>0$ para

$$
\|x\|_{Y} \leq \epsilon\|A x\|+K \epsilon^{-\alpha /(1-\alpha)}\|x\|,
$$

para todo $\epsilon, \epsilon>0$.

Então, sempre que $\beta \in(\alpha, 1]$,

$$
X_{\beta, 1 ; \infty}^{\prime}(A) \subset Y \text {. }
$$

com constante de imersão não dependente de $A \in \mathcal{A} e$

$$
\left(X, X_{1}\right)_{\beta, \infty ; K} \subset Y \text {. }
$$


Prova. Suponha $f \in X_{\beta, 1 ; \infty}^{\prime}(A)$. Obviamente $f=f-e^{-A} f+e^{-A} f$. Por ser $e^{-s A}$ semigrupo analítico,

$$
f=\lim _{n \rightarrow+\infty}\left[\int_{1 / n}^{1} A e^{-s A} f d s+e^{-A} f\right],
$$

novamente por [Butzer e Berens[3], relação 2.3.1, página 111].

Chame a soma entre conchetes de $y_{n}$. Como $y_{n}=e^{-1 / n A} f$ então $y_{n} \in$ $D(A)$ e, portanto, $y_{n} \in Y$. Portanto, para demonstrar que $f \in Y$, é suficiente verificar que $\left(y_{n}\right)$ é uma sequência de Cauchy em $Y$. Realmente, neste caso, por ser $Y$ espaço de Banach, $y_{n} \rightarrow g$ na norma de $Y$, algum $g \in Y$. Mas, por construção, $y_{n} \rightarrow f$ na norma de $X$. Como $Y \subset X$ continuamente, $f=g$.

Pelo lema 1.2.29 abaixo e constante $c$ aí definida, para $n \leq m$,

$$
\left\|y_{n}-y_{m}\right\|_{Y} \leq\left\|\int_{1 / m}^{1 / n} A e^{-s A} f d s\right\|_{Y} \leq c \frac{(1 / n)^{\beta-\alpha}-(1 / m)^{\beta-\alpha}}{\beta-\alpha}\|f\|_{\beta, 1 ; \infty}^{\prime} .
$$

Em qualquer caso,

$$
\left\|y_{n}-y_{m}\right\|_{Y} \leq c \frac{\left|(1 / n)^{\beta-\alpha}-(1 / m)^{\beta-\alpha}\right|}{\beta-\alpha}\|f\|_{\beta, 1 ; \infty}^{\prime}
$$

e $\left(y_{n}\right)$ é mesmo uma sequência de Cauchy em $Y$.

Para ver a continuidade da imersão de $X_{\beta, 1 ; \infty}^{\prime}(A)$ em $Y$, note que, devido ao lema 1.2.29 e à proposição 1.2.19,

$$
\left\|\int_{1 / n}^{1} A e^{-s A} f d s+e^{-A} f\right\|_{Y} \leq \max \left\{c \frac{1-(1 / n)^{\beta-\alpha}}{\beta-\alpha}, C c_{1}^{\alpha} c_{0}^{1-\alpha} e^{-\delta}\right\}\|f\|_{\beta, 1 ; \infty}
$$

Tomando o limite quando $n \rightarrow \infty$,

$$
\|f\|_{Y} \leq \max \left\{c \frac{1}{\beta-\alpha}, C c_{1}^{\alpha} c_{0}^{1-\alpha} e^{-\delta}\right\}\|f\|_{\beta, 1 ; \infty}
$$

A independência da constante de imersão com $A$ segue da parte 2 do teorema 1.2.20.

Lema 1.2.29 Com as mesmas hipóteses do teorema 1.2.28, se $0<a \leq b$,

$$
\left\|\int_{a}^{b} A e^{-s A} f d s\right\|_{Y} \leq \frac{b^{\beta-\alpha}-a^{\beta-\alpha}}{\beta-\alpha} \sup _{<s<\infty}\left(s^{1-\beta}\left\|A e^{-s A} f\right\|\right)
$$

onde $c=2^{1+\alpha-\beta} C c_{0}^{1-\alpha} c_{1}^{\alpha}$, a constante $C$ definida no próprio teorema e $c_{0}, c_{1}$ como na proposição 1.2.19. 
Prova. Antes de mais nada, avaliemos $A e^{-s A} f$ na norma de $Y$.

Pelas propriedades de semigrupo, $A e^{-s A} f=e^{-s / 2 A} A e^{-s / 2 A} f$ e $A^{2} e^{-s A} f=$ $A e^{-s / 2 A} A e^{-s / 2 A} f$. Portanto, pela proposição 1.2 .19 e pela hípótese do teorema,

$$
\left\|A e^{-s A} f\right\|_{Y} \leq C\left(c_{0}\right)^{1-\alpha}\left(2 c_{1}\right)^{\alpha} \frac{e^{-s / 2 \delta}}{s^{\alpha}}\left\|A e^{-s A / 2} f\right\| .
$$

Notando que $\left\|A e^{-s A / 2} f\right\|=(s / 2)^{\beta-1}(s / 2)^{1-\beta}\left\|A e^{-s A / 2} f\right\|$, sendo $c$ como no enunciado, a prova pode ser concluída com

$$
\left\|\int_{a}^{b} A e^{-s A} f d s\right\|_{Y} \leq c \int_{a}^{b} s^{\beta-\alpha-1}(s / 2)^{1-\beta}\left\|A e^{-s A / 2} f\right\| d s
$$

Regularidade dos espaços no caso $X=L_{p}$ e $D(A) \subset W_{m, p}$

Proposição 1.2.30 Suponha $\Omega \subset \mathbb{R}^{n}$ um conjunto fronteira Lipschitz quando $\Omega$ é limitado ou possui a propriedade de extensão $C^{m}$ quando $\Omega$ não é limitado[ Henry[8],página 38]. Suponha $A \in \mathcal{A}, \mathcal{A}$ como em $1.2 .13 \mathrm{com}$ $X=L_{p}(\Omega), 0<p<\infty$ com $D(A) \subset W^{m, p}(\Omega)$ continuamente, $m \geq 1$. $A \in \mathcal{A}$,

Então, para $\alpha \in(0,1)$, com constantes de imersão não dependentes de

1. $X_{\alpha, 1 ; \infty}^{\prime}(A) \subset W^{k, q}(\Omega)$ quando $k-n / q<m \alpha-n / p, q \geq p$.

2. $X_{\alpha, 1 ; \infty}^{\prime}(A) \subset C^{\nu}(\Omega)$ quando $0 \leq \nu<m \alpha-n / p$.

Prova. Pela Desigualdade de Niremberg-Gaglilardo [Henry[8], página 37, a]

$$
\|u\|_{W^{k, p}(\Omega)} \leq C\|u\|_{W^{m, p}(\Omega)}^{\theta}\|u\|_{L_{p}(\Omega)}^{1-\theta}
$$

contanto que $k-n / q \leq \theta(m-n / p)-n(1-p)=m \theta-n / p$ e $q \geq p$. Para $u \in D(A)$, uma vez que $D(A) \subset\|u\|_{W^{m, p}}(\Omega)$ e $\left\|A^{-1}\right\| \leq C_{\mathcal{A}}$ por 1.2.13,

$$
\|u\|_{W^{k, p}(\Omega)} \leq C_{1}\|A u\|_{L_{p}(\Omega)}^{\theta}\|u\|_{L_{p}(\Omega)}^{1-\theta} .
$$

Por 1.2.28, com $Y=W^{k, p}(\Omega), X=L_{p}(\Omega)$, tomando $\alpha>\theta$, concluímos a prova de 1.

A parte 2 é provada similarmente, aplicando também uma desigualdade de Nirenberg-Gagliardo[Henry[8], página 37, b]

$$
\|u\|_{C^{\nu}} \leq C\|u\|_{W^{m, p}}^{\theta}\|u\|_{L_{p}}^{1-\theta}
$$

se $0 \leq \theta \leq 1,0 \leq \nu \leq \theta(m-n / p)-n(1-\theta) / p=m \theta-n / p$. 
Observação 1.2.31 Chame $X=X_{0}$. Os espaços $\left(X_{0}, X_{1}\right)_{\alpha, \infty ; K}$ são muito bons para trabalhar com sistemas de equações. Digamos que um operador de um sistema tem dominio $X_{1}=W_{n}^{2, p}(\Omega) \times W_{n}^{2, p}(\Omega)$ e tem valores em $X_{0}=L_{p}(\Omega) \times L_{p}(\Omega)$, onde as notações se referem aos espaços de Sobolev $e$ o $n$ indica que os elementos também tem derivada na direção normal a $\partial \Omega$ nula.

Para descrever $\left(X_{0}, X_{1}\right)_{\alpha, \infty ; K}$, escolho um operador $A$ em $X_{0}$ que tem dominio $X_{1}$ e $A \in \mathcal{A}, \mathcal{A}$ como em 1.2.13. Uma boa escolha é

$$
A=\operatorname{dig}\left(-\Delta_{n}+c I,-\Delta_{n}+c I\right),
$$

com $-\Delta_{n}$ o Laplaciano em $W_{n}^{2, p}$. Então se c é grande o suficiente, $\left(-\Delta_{n}+\right.$ $c I)^{-1}: L_{p} \rightarrow W_{n}^{2, p}$ existe e é limitado em $L_{p}$ com $\sigma(A)>0$ e $A \in \mathcal{A}$.

Não há dúvida que $X_{1} \subset D(A)$. Para ver que $D(A) \subset X_{1}$, seja $u \in D(A)$, $u=\left(u^{1}, u^{2}\right)^{t}$. Por estimativas a priori para um operador eliptico que tem a derivada normal na fronteira de $\Omega$ nula, $\left\|u^{1}\right\|_{W^{2, p}} \leq\left\|\left(-\Delta_{n}+c I\right) u^{1}\right\|$. Uma referência sobre teoremas com estimativas para operadores elíticos pode ser [[15],teorema 3.1.1, página 71]. Analogamente, para $u^{2}$. Então, $\|u\|_{X_{1}} \leq$ $c\|A u\|_{L_{p} \times L_{p}}$.

Pela desiguladade de Niremberg-Gagliardo [[8], página 37, a], com $k, q, \theta$ convenientes,

$$
\left\|u^{1}\right\|_{W^{k, q}(\Omega)} \leq C\left\|u^{1}\right\|_{W^{2, p}}^{\theta}\left\|u^{1}\right\|_{L_{p}}^{1-\theta}
$$

e analogamente para $u^{2}$. Então, escolhendo, por facilidade, para os espaços produto, a norma do max,

$$
\|u\|_{W^{k, q} \times W^{k, q}} \leq C\|u\|_{W^{2, p} \times W^{2, p}}^{\theta}\|u\|_{L_{p} \times L_{p}}^{1-\theta}
$$

e então

$$
\|u\|_{W^{k, q} \times W^{k, q}} \leq C\|A u\|_{L_{p} \times L_{p}}^{\theta}\|u\|_{L_{p} \times L_{p}}^{1-\theta} .
$$

Aplicaşão do teorema 1.2.28, implica $\left(X_{0}, X_{1}\right)_{\alpha, \infty ; K} \subset W^{k, p}(\Omega) \times W^{k, p}(\Omega)$ se $\alpha>\theta$. Ou precisamente, a imersão ocorre se $q \geq p$ e $k-n q<2 \alpha-n / p$.

Com o mesmo tipo de argumento, obtém-se $\left(X_{0}, X_{1}\right)_{\alpha, \infty ; K} \subset C^{\nu}(\Omega) \times$ $C^{\nu}(\Omega)$ quando $0 \leq \nu<2 \alpha-n / p$.

Observação 1.2.32 Sejam $X_{0}, X_{1}$ espaços de Banach, com $X_{1} \subset X_{0}$ densa e continuamente. Suponha que existe um conjunto $\mathcal{A}=\{A\}, \mathcal{A}$ como em $1.2 .13 \mathrm{em}$ que $X=X_{0}$. Admita que $D(A) \doteq X_{1}$. Portanto o elementos de 
$\left(X_{0}, X_{1}\right)_{\alpha, \infty ; K}=\left(X_{0}, D(A)\right)_{\alpha, 1 ; \infty} e$, para o caso $X_{0}=L_{p}(\Omega)$ e $X_{1}=W_{0}^{2, p}(\Omega)$ nas hipóteses do teorema $1.2 .30 \mathrm{com} \alpha$ conveniente, sẫo funções $C(\bar{\Omega})$.

Para um exemplo explícito, seja $A(u) v=-g(u) \Delta v+$ av, com $D(A(u))=$ $W_{0}^{2,2}(\Omega)$ e $g \in C(\mathbb{R})$ e $a>0$. Notemos que se $X_{0}=L_{2}(\Omega)$ e $X_{1}=W_{0}^{2,2}(\Omega)$, tomando, por exemplo, $B=-\Delta \operatorname{com} D(B)=W_{0}^{2,2}(\Omega)$, temos $D(B) \doteq X_{1}$, e então $\left(X_{0}, X_{1}\right)_{\alpha, \infty ; K}^{\circ}$ está imerso no conjunto $C(\bar{\Omega})$. Então é possivel achar condições para que o conjunto $\mathcal{A}=\left\{A(u), u \in\right.$ limitado de $\left.\left(X_{0}, X_{1}\right)_{\alpha, \infty ; K}\right\}$ seja um conjunto regular de $\mathcal{H}\left(X_{1}, X_{0}\right)$. Por exemplo, uma condição suficiente pode ser achada em [[15],teorema 3.1.3, página 73], que garante que exite $a_{0}>0$ para que $A(u) \in \mathcal{H}\left(X_{1}, X_{0}\right)$ se $a \geq a_{0}$. A referência [[1], seção 3, página 54] fornece uma prova simples para isto se gé $C^{1}(\mathbb{R})$ e $\alpha>0$ escolhido para que os elementos de $\left(X_{0}, X_{1}\right)_{\alpha, \infty ; K}=\left(X_{0}, D(A)\right)_{\alpha, 1 ; \infty}$ sejam funções de $C^{1}(\bar{\Omega})$.

\subsubsection{Espaços $X_{\alpha, 1 ; \infty}^{o}$ e $(X, D(A))_{\alpha, \infty ; K}^{o}$}

Os espaços $X_{\alpha, 1 ; \infty}^{\prime}$ previamente definidos possuem um defeito grave. Não é garantido que em geral o semigrupo $e^{-s A}, A \in \mathcal{A}, \mathcal{A}$ como em 1.2.13 é fortemente contínuo em $X_{\alpha, 1 ; \infty}^{\prime}$. Ou seja, não é garantido que

$$
\left\|\left(e^{-t A}-I\right) f\right\|_{\alpha, 1 ; \infty} \rightarrow 0
$$

quando $t \rightarrow 0+$ se $f \in X_{\alpha, 1 ; \infty}^{\prime}$. Esta, digamos, perda de continuidade no 0 pode ser um inconveniente nas aplicações às EDP's. Mas o fato pode ser remediado trabalhando com os espaços $X_{\alpha, 1 ; \infty}^{o}$ que é o subespaço de $X_{\alpha, 1 ; \infty}^{\prime}$ garantindo continuidade no 0 . Melhor ainda, para garantir continuidade no 0 uniformemente com $A$, recorremos aos espaços $(X, D(A))_{\alpha, 1 ; \infty}^{o}$ definidos a seguir.

Definição 1.2.33 Seja $A \in \mathcal{A}, \mathcal{A}$ como em 1.2.13. O subespaço formado pelas $f \in X_{\alpha, 1 ; \infty}^{\prime}(A)$ com a mesma norma e que obedecem

$$
\left\|\left(e^{-t A}-I\right) f\right\|_{\alpha, 1 ; \infty} \rightarrow 0
$$

quando $t \rightarrow 0+$ é chamado de $X_{\alpha, 1 ; \infty}^{o}(A)$.

Proposição 1.2.34 Seja $\alpha \in[0,1]$. O espaço $X_{\alpha, 1 ; \infty}^{o}(A)$ é um espaço de $B a$ nach que contém $D(A)$ como conjunto denso. E também $D(A) \doteq X_{1,1 ; \infty}^{o}(A)$, $X_{0,1 ; \infty}^{o}(A) \doteq X_{0,1 ; \infty}(A) \doteq X$. 
Prova. Para obter $D(A) \subset X_{\alpha, 1 ; \infty}^{o}(A)$ é só observar que, para $f \in D(A)$,

$$
\left\|\left(e^{-t A}-I\right) f\right\|_{\alpha, 1 ; \infty}^{\prime}=\left\|\left(e^{-t A}-I\right) f\right\|+\sup _{0<s<\infty}\left(s^{1-\alpha}\left\|e^{-s A}\left(e^{-t A}-I\right) A f\right\|\right)
$$

e aplicar 1.2 .19 e o fato de que $e^{-t A}$ ser fortemente contínuo e $\sup _{0<s<\infty}\left\{s^{1-\alpha} e^{-\delta s}\right\}$ ser finito.

O fato de $D(A)$ ser denso está incluído na definição, pois $e^{-t A} f \in D(A)$ se $t>0$. Com isto e também com 2.1.1 escolhendo $\beta=\alpha$, é possível concluir que $X_{\alpha, 1 ; \infty}^{o}(A)$ é um subespaço fechado de $X_{\alpha, 1 ; \infty}^{\prime}(A)$, portanto, espaço de Banach.

Para acabar, note que as normas de $X_{1,1 ; \infty}^{\prime}(A)$ e $D(A)$ são equivalentes, pois, se $f \in D(A)$,

$$
\|f\|_{\alpha, 1 ; \infty}^{\prime} \leq\|f\|+\sup _{0<s<\infty}\left(\left\|e^{-s A} A f\right\|\right)
$$

e, também, por ser $X_{1,1 ; \infty}^{\prime}(A)$ espaço intermediário entre $D(A)$ e $X, D(A) \subset$ $X_{1,1 ; \infty}^{\prime}(A)$. Consequentemente se $f \in X_{1,1 ; \infty}^{o}(A),\left(f_{n}\right) \subset D(A)$, e $f_{n} \rightarrow f$ na norma de $X_{1,1 ; \infty}^{\prime}(A)$, o mesmo se dá na norma de $D(A)$. Mas $D(A)$ é um espaço de Banach e, então, $f \in D(A)$.

Vimos em 1.2.27 que $X_{0,1 ; \infty}(A) \doteq X$, então $X_{0,1 ; \infty}^{o}(A) \doteq X$, pois $e^{-s A}$ é semigrupo fortemente contínuo em $X$.

Proposição 1.2.35 Suponha $A \in \mathcal{A}, A$ como em 1.2.13 e $K$ como em 1.2.5. Então, para $f \in X$, supondo lado direito das desiguadades finito,

1. Parat $>0$ e $\alpha \in(0,1]$,

$$
\left\|\left(e^{-t A}-I\right)\right\| \leq t^{\alpha} / \alpha \sup _{0<s \leq t}\left(s^{1-\alpha}\left\|A e^{-s A} f\right\|\right) .
$$

2. Para $t>0$ e $\alpha \in(0,1]$,

$$
\left\|\left(e^{-t A}-I\right) f\right\|_{X_{\alpha, 1 ; \infty}^{\prime}(A)} \leq c \sup _{0<s \leq t}\left(s^{1-\alpha}\left\|A e^{-s A} f\right\|\right)
$$

$\operatorname{com} c=\max \left\{c_{0}+1, c_{1} / \alpha, t^{\alpha} / \alpha\right\}$.

3. Parat $>0$ e $\alpha \in \mathbb{R}$,

$$
t^{1-\alpha}\left\|A e^{-t A} f\right\| \leq \max \left\{c_{0}, c_{1}\right\} t^{-\alpha} K(t, f, X, D(A)) .
$$


4. Para $t>0$ e $\alpha>0$,

$$
\begin{aligned}
& t^{-\alpha} K(t, f, X, D(A)) \\
& \quad \leq(1+1 / \alpha) \sup _{0<s \leq t}\left(s^{1-\alpha}\left\|A e^{-s A} f\right\|\right)+c_{0} e^{-\delta t} t^{1-\alpha}\|f\| .
\end{aligned}
$$

5. $\operatorname{Para} A, B \in \mathcal{A}, t>0$,

$$
t^{-\alpha} K(t, f, X, D(A)) \leq c t^{-\alpha} K(t, f, X, D(B)),
$$

com constante $c$ independente do par $A, B$.

Prova. Se $t>0,1$ segue diretamente de

$$
\left(e^{-t A}-I\right) f=e^{-t A} f-\lim _{\epsilon \rightarrow 0} e^{-\epsilon A} f=-\lim _{\epsilon \rightarrow 0} \int_{\epsilon}^{t} A e^{-s A} f d s .
$$

Para um $t>0$, suponha $\sup _{0<s \leq t}\left(s^{1-\alpha}\left\|A e^{-s A} f\right\|\right)$ finito.

Para avaliação de

$$
\begin{aligned}
& \left\|\left(e^{-t A}-I\right)\right\|_{X_{\alpha, 1 ; \infty}^{\prime}(A)} \\
& \quad=\left\|\left(e^{-t A}-I\right) f\right\|+\sup _{0<s<\infty}\left(s^{1-\alpha}\left\|A e^{-s A}\left(e^{-t A}-I\right) f\right\|\right),
\end{aligned}
$$

falta analisar a segunda parcela, em virtude de 1 .

Para avaliá-la, separamos a análise em dois casos. De um lado, comutando,

$$
\begin{aligned}
& \sup _{0<s \leq t}\left(s^{1-\alpha}\left\|A e^{-s A}\left(e^{-s A}-I\right) f\right\|\right) \\
& \quad \leq\left\|e^{-t A}-I\right\| \sup _{0<s \leq t}\left(s^{1-\alpha}\left\|A e^{-s A} f\right\|\right)
\end{aligned}
$$

$\mathrm{e}\left\|e^{-t A}-I\right\| \leq c_{0}+1$. Do outro lado, como $\left\|A e^{-s A}\right\| \leq c_{1} / s \mathrm{e}$ $s^{-\alpha}<t^{-\alpha}$ se $t<s$,

$$
\sup _{t<s<\infty}\left(s^{1-\alpha}\left\|A e^{-s A}\left(e^{-t A}-I\right) f\right\|\right) \leq c_{1} t^{-\alpha}\left\|\left(e^{-t A}-I\right) f\right\| .
$$

Também com aplicação de 1 ,

$$
\begin{aligned}
& \sup _{0<s<\infty}\left(s^{1-\alpha}\left\|A e^{-s A}\left(e^{-t A}-I\right) f\right\|\right) \\
& \quad \leq \max \left\{c_{0}+1, c_{1} / \alpha\right\} \sup _{0<s \leq t}\left(s^{1-\alpha}\left\|A e^{-s A} f\right\|\right)
\end{aligned}
$$


o que leva a 2 .

$\mathrm{O}$ item 3 é uma consequência óbvia de 1.2.22.

Para provar 4, seja a um elemento de $D(A)$ e $x$ um elemento de $X$. Portanto, para $t>0$,

$$
\begin{gathered}
K(t, f, X, D(A))=\inf _{f=x+a}(\|x\|+t\|a\|+t\|A a\|) \\
\leq\left\|\left(I-e^{-t A}\right) f\right\|+t\left\|e^{-t A} f\right\|+t\left\|A e^{-t A} f\right\|,
\end{gathered}
$$

pois $\left(I-e^{-t A}\right) f \in X$ e $e^{-t A} f \in D(A)$. Portanto para obter 4, é só aplicar 1 e 1.2.19.

Para ver 5 , é suficiente relembrar a definição de $\mathcal{A}$ e $K$.

Definição 1.2.36 Seja $\alpha \in[0,1]$. Para $A \in \mathcal{A}, \mathcal{A}$ como em 1.2.13, definimos o subespaço de $X$

$$
\begin{aligned}
& \operatorname{Lip}(\alpha, X, D(A)) \\
& \quad:=\left\{f \in X, t^{1-\alpha}\left\|A e^{-t A} f\right\| \rightarrow 0 \text { se } t \rightarrow 0+\right\} .
\end{aligned}
$$

Proposição 1.2.37 $O$ espaço $\operatorname{Lip}(\alpha, X, D(A))$ é subespaço linear fechado de $X_{\alpha, 1 ; \infty}(A)$, portanto, espaço de Banach se $\alpha \in[0,1]$ e, para $\alpha \in[0,1)$, $\operatorname{Lip}(\alpha, X, D(A)) \doteq X_{\alpha, 1 ; \infty}^{o}(A)$ com normas iguais. Em particular, Lip $(0, X, D(A))$ $\doteq X$. Para $\alpha=1, \operatorname{Lip}(1, X, D(A)) \subset X_{1,1 ; \infty}^{o}(A)$.

Prova. Suponha $\alpha \in[0,1]$.

O espaço $X_{\alpha, 1 ; \infty}(A)$ contém $\operatorname{Lip}(\alpha, X, D(A))$, pois

$$
\begin{aligned}
& \sup _{0<s<\infty}\left(s^{1-\alpha}\left\|A e^{-s A} f\right\|\right) \\
& \quad \leq \max \left\{\sup _{0<s<\delta}\left(s^{1-\alpha}\left\|A e^{-s A} f\right\|\right), \sup _{s \geq \delta}\left(s^{1-\alpha}\left\|A e^{-s A} f\right\|\right)\right\}
\end{aligned}
$$

e, para $f \in \operatorname{Lip}(\alpha, X, D(A))$, o último majorante não é maior que, digamos, $\max \left\{1, c_{1} \delta^{-\alpha}\right\}$ se escolhemos $\delta>0$ para $\sup _{0<s<\delta}\left(s^{1-\alpha}\left\|A e^{-s A} f\right\|\right)<1$. Não há dúvida que $\operatorname{Lip}(\alpha, X, D(A))$ é linear.

Para ver que $\operatorname{Lip}(\alpha, X, D(A))$ é um subespaço fechado, suponha então $\left(f_{n}\right) \subset \operatorname{Lip}(\alpha, X, D(A))$ uma sequência com limite $f$ na norma de $X_{\alpha, 1 ; \infty}^{\prime}(A)$. Obviamente $A e^{-t A} f=A e^{-t A}\left(f-f_{n}\right)+A e^{-t A} f_{n}$ e, portanto,

$$
\begin{aligned}
& t^{1-\alpha}\left\|A e^{-t A} f\right\| \\
& \quad \leq \sup _{0<s<\infty}\left(s^{1-\alpha}\left\|A e^{-s A}\left(f-f_{n}\right)\right\|\right)+t^{1-\alpha}\left\|A e^{-s A} f_{n}\right\| .
\end{aligned}
$$


Dado $\epsilon>0$, escolhemos $n_{0}$ para $\left\|f-f_{n_{0}}\right\|_{X_{\alpha, 1 ; \infty}^{\prime}(A)}<\epsilon / 2$, e, em seguida, $\delta>0$, para $t^{1-\alpha}\left\|A e^{-s A} f_{n_{0}}\right\|<\epsilon / 2$ se $0<t<\delta$. Portanto também $t^{1-\alpha}\left\|A e^{-s A} f\right\|<\epsilon$ se $0<t<\delta$ e concluímos que $f \in \operatorname{Lip}(\alpha, X, D(A))$.

Quando $\alpha \in(0,1]$, por 2 de 1.2.35, $\operatorname{Lip}(\alpha, X, D(A)) \subset X_{\alpha, 1 ; \infty}^{\circ}(A)$. Também $D(A) \subset \operatorname{Lip}(\alpha, X, D(A))$ se $\alpha \in[0,1)$, pois se $f \in D(A), t^{1-\alpha}\left\|A e^{-t A} f\right\| \leq$ $c_{0} t^{1-\alpha}\|A f\|$.

Também para $\alpha=0$, vimos acima, $\operatorname{Lip}(\alpha, X, D(A)) \subset X_{\alpha, 1 ; \infty}(A)$, mas, por 1.2.34, $X_{\alpha, 1 ; \infty}(A) \doteq X_{\alpha, 1 ; \infty}^{o}(A) \doteq X$.

Para os casos $\alpha \in[0,1)$, obtemos, portanto, $D(A) \subset \operatorname{Lip}(\alpha, X, D(A)) \subset$ $X_{\alpha, 1 ; \infty}^{o}(A)$ e tomando o fecho, como $\overline{D(A)}=X_{\alpha, 1 ; \infty}^{o}(A)$, obtemos $\operatorname{Lip}(\alpha, X, D(A))=$ $X_{\alpha, 1 ; \infty}^{o}(A)$.

Definição 1.2.38 Sejam $A \in \mathcal{A}, \mathcal{A}$ como em 1.2.13 e $K$ como em 1.2.5. $O$ espaço formado pelas $f \in X$ que obedecem

$$
t^{-\alpha} K(t, f, X, D(A)) \rightarrow 0 \text { se } t \rightarrow 0
$$

$K$ como em 1.2.5, será denotado por $(X, D(A))_{\alpha, \infty ; K}^{\circ}$.

Proposição 1.2.39 Seja $\alpha \in(0,1)$. O espaço $(X, D(A))_{\alpha, \infty ; K}^{o}$ é um espaço de Banach contido em $(X, D(A))_{\alpha, \infty ; K}$ com igual norma $e(X, D(A))_{\alpha, \infty ; K}^{o} \doteq$ $\operatorname{Lip}(\alpha, X, D(A)) \doteq X_{\alpha, 1 ; \infty}^{o}(A)$.

Prova. Registre que, pela 1.2.37, Lip $(\alpha, X, D(A)) \doteq X_{\alpha, 1 ; \infty}^{o}(A)$. Por 3 de 1.2 .35 e 1 de $1.2 .20,(X, D(A))_{\alpha, \infty ; K}^{\circ} \subset \operatorname{Lip}(\alpha, X, D(A))$ e por 4 de $1.2 .35 \mathrm{e}$ 1 de 1.2.20, $\operatorname{Lip}(\alpha, X, D(A)) \subset(X, D(A))_{\alpha, \infty ; K}^{o}$, ambas imersões contínuas, concluindo a prova.

Proposição 1.2.40 Seja $\alpha \in(0,1)$. Se $f \in(X, D(A))_{\alpha, \infty ; K}^{o}$ para algum $A \in \mathcal{A}$ então $\left\|\left(e^{-t B}-I\right) f\right\|_{X_{\alpha, 1 ; \infty}^{\prime}(C)} \rightarrow 0+$ uniformemente para $B, C \in \mathcal{A}$.

Prova. Para $\epsilon>0$ arbitrário, escolhamos $0<\delta<1$ para que se $0<t<\delta$ então

$$
t^{-\alpha} K(t, f, X, D(A)) \leq \epsilon /\left(a_{1} a_{2} a_{3}\right)
$$

com constantes $a_{1}, a_{2}, a_{3}$ descritas abaixo. 


\subsection{RELAÇÃO COM OS ESPAÇOS DE POTÊNCIA FRACIONÁRIA 33}

Seja $a_{1}$ para

$$
t^{-\alpha} K(t, f, X, D(B)) \leq a_{1} t^{-\alpha} K(t, f, X, D(A))
$$

uniformemente com $B \in \mathcal{A}$, garantida por 3 , de 1.2.35.

Também por 2 de 1.2 .35 , existe $a_{2}$ para

$$
\left\|\left(e^{-t B}-I\right) f\right\|_{X_{\alpha, 1 ; \infty}^{\prime}(B)} \leq a_{2} \sup _{0<s \leq t}\left(s^{1-\alpha}\left\|s^{1-\alpha} B e^{-s B} f\right\|\right) .
$$

Explicitamente, como $0<t<\delta<1, a_{2}=\max \left\{c_{0}+1, c_{1} / \alpha, 1 / \alpha\right\}$.

Por último, seja $a_{3}$, a constante de imersão $X_{\alpha, 1 ; \infty}^{\prime}(B) \subset X_{\alpha, 1 ; \infty}^{\prime}(C)$, que não depende de $B, C \in \mathcal{A}$, por 1.2.20.

Partindo de

$$
\left\|\left(e^{-t B}-I\right) f\right\|_{X_{\alpha, 1 ; \infty}^{\prime}(C)} \leq a_{3}\left\|\left(e^{-t B}-I\right) f\right\|_{X_{\alpha, 1 ; \infty}^{\prime}(B)}
$$

e com aplicação de $2,3,5$ de 1.2 .35 , obtemos $\left\|\left(e^{-t B}-I\right) f\right\|_{X_{\alpha, 1 ; \infty}^{\prime}(C)} \leq \epsilon$ sempre que $0<t<\delta$.

\subsection{Relação com os espaços de potência fra- cionária}

Originalmente, procurávamos um substituto para os espaços de potências com norma $\left\|A^{\alpha} f\right\|$ que o professor Daniel Henry [Henry[8]] usou para descrever as soluções da equação parabólica semilinear que pudesse descrever as soluções de equações parabólicas possivelmente não semilineares. A primeira escolha recaia sobre os espaços $X_{\alpha, 1 ; \infty}^{\prime}(A)$. Como previamente discutido, o semigrupo $e^{-t A}$ não é necessariamente fortemente contínuo nem o domínio $D(A)$ necessariamente denso neste espaço. Então partimos para o substituto $X_{\alpha, 1 ; \infty}^{o}(A)$ que tem estas vantagens.

$\mathrm{O}$ teorema seguinte mostra um relacionamente entre eles.

Teorema 1.3.1 Suponha $\mathcal{A}$ como em 1.2.13. Então se $0 \leq \alpha \leq \alpha+\epsilon \leq 1$

$$
X_{\alpha+\epsilon, 1 ; \infty}^{o}(B) \subset X^{\alpha}(A) \subset X_{\alpha, 1 ; \infty}^{o}(C),
$$

arbitráriamente para $A, B, C$ em $\mathcal{A}$ com imersões com constantes que não dependem dos operadores usados e também densas. Especificamente se $A=$ $B=C$, as constantes para primeira e segunda imersões são respectivamente $2^{1-\alpha-\epsilon} c_{\alpha} /(1-\epsilon)$ e $\max \left\{\frac{e}{e-1} c_{1-\alpha} \frac{\left(c_{0} / \delta\right)^{\alpha}}{\alpha}, c_{1-\alpha}\right\} \operatorname{com} c_{\alpha}=2(2 \alpha)^{-\alpha} c_{0}^{2-\alpha} c_{1}^{\alpha} / \Gamma(2-$ $\alpha)$. No caso particular $\alpha=0, X^{o}(A) \doteq X_{0,1 ; \infty}^{o}(B) \doteq X$. 
Prova. É suficiente fazer a prova para $A=B=C$ devido a 1.2.20.

Como $D(A) \subset X_{\alpha+\epsilon, 1 ; \infty}^{o}(A)$ densamente pela proposição 1.2.34, existe uma sequência $\left(f_{n}\right) \operatorname{com~} f_{n} \rightarrow f$ em $X_{\alpha+\epsilon, 1 ; \infty}(A)$. Para concluir que $f \in$ $X^{\alpha}(A)$, é suficiente provar que $\left(f_{n}\right)$ é uma sequência de Cauchy em $X^{\alpha}(A)$. Vendo que $f_{m}-f_{n}=-\left(e^{-A}-I\right)\left(f_{m}-f_{n}\right)+e^{-A}\left(f_{m}-f_{n}\right)$

$$
\left\|A^{\alpha}\left(f_{m}-f_{n}\right)\right\| \leq\left\|\int_{0}^{1} A^{\alpha} A e^{-s A}\left(f_{m}-f_{n}\right) d s\right\|+\left\|A^{\alpha} e^{-A}\left(f_{n}-f_{m}\right)\right\| .
$$

Com a substuição $1=(s / 2)^{\alpha+\epsilon-1}(s / 2)^{1-\alpha-\epsilon}$ e com a estimativa $\left\|A e^{-s A}\right\| \leq$ $c_{\alpha} s^{-\alpha} e^{-s \delta}$, sendo $c_{\alpha}=2(2 \alpha)^{-\alpha} c_{0}^{2-\alpha} c_{1}^{\alpha} / \Gamma(2-\alpha)$ [Henry[8], páginas 26 e 27],

$$
\left\|A^{\alpha}\left(f_{m}-f_{n}\right)\right\| \leq 2^{1-\alpha-\epsilon} \frac{c_{\alpha}}{1-\epsilon}\left\|f_{m}-f_{n}\right\|_{X_{\alpha+\epsilon, 1 ; \infty}^{\prime}(A)} .
$$

Portanto, $\left(f_{n}\right)$ é sequência de Cauchy em $X^{\alpha}(A)$ que tem limite $f$ em $X^{\alpha}(A)$. Repetindo o mesmo cálculo com $f_{n}$ no lugar de $f_{n}-f_{m}$ e tomando o limite quando $n \rightarrow \infty$, obtém-se a primeira imersão do teorema.

Prossegindo, suponha que $f \in X^{\alpha}(A)$. Para avaliar a norma em $X$, se $\alpha>0$, vendo que $f=-\left(e^{-c_{0} A / \delta}-I\right) f+e^{-c_{0} A / \delta} f$,

$$
f=\lim _{\mu \rightarrow 0+} \int_{\mu}^{c_{0} / \delta} A e^{-s A} f d s+e^{-c_{0} A / \delta} f .
$$

Portando $\|f\| \leq c_{1-\alpha}\left(c_{0} / \delta\right)^{\alpha} / \alpha\left\|A^{\alpha} f\right\|+1 / e\|f\| \mathrm{e}$

$$
\|f\| \leq \frac{e}{e-1} c_{1-\alpha} \frac{\left(c_{0} / \delta\right)^{\alpha}}{\alpha}\left\|A^{\alpha} f\right\| .
$$

Para avaliar $\sup _{0<s<\infty}\left(s^{1-\alpha} A e^{s A} f\right)$, fazemos a permutação $A e^{s A} f=A^{1-\alpha} e^{s A} A^{\alpha} f$, para obter

$$
\sup _{0<s<\infty}\left(\| s^{1-\alpha} A e^{-s A} f\right)\left\|\leq c_{1-\alpha}\right\| A^{\alpha} f \| .
$$

Com isto, $X^{\alpha}(A) \subset X_{\alpha, 1 ; \infty}^{\prime}(A)$ com constante de imersão $\max \left\{\frac{e}{e-1} c_{1-\alpha} \frac{\left(c_{0} / \delta\right)^{\alpha}}{\alpha}, c_{1-\alpha}\right\}$.

Mas $A^{\alpha}\left(e^{-t A}-I\right) f=\left(e^{-t A}-I\right) A^{\alpha} f$ e então $\left\|A^{\alpha}\left(e^{-t A}-I\right) f\right\| \rightarrow 0$ se $t \rightarrow 0+$. Pela imersão que acabamos de provar, $\left\|\left(e^{-t A}-I\right) f\right\|_{X_{\alpha, 1 ; \infty}^{\prime}(A)} \rightarrow 0+$ também se $t \rightarrow 0+$, o que implica que vale a segunda imersão.

O fato de que todas a imersões são densas segue de que $D(A)$ é um subespaço denso de todos os espaços do teorema, conforme 1.2.34 e [Henry[8], página 25].

A última afirmação pode ser concluída da proposição 1.2 .34 e porque $X^{\circ}(A)=X$ pela [Henry[8], página 25, definição 1.4.1]. 


\subsection{Conexão com os resultados de Grisvard}

É possível obter uma descrição explícita para os espaços intermediários dos tópicos anteriores para alguns operadores e domínios conforme descrição em, por exemplo, [[17]].

É necessário antes mostrar que os espaços até aqui vistos se igualam aos espaços definidos em Grisvard [17].

Definição 1.4.1 Suponha que $X$ é um espaço de Banach e A um operador linear fechado com domínio $D(A) \subset X$, densamente. Suponha que $-A$ gera um semigrupo fortemente continuo $e^{-t A}$, uniformemente limitado, com $\left\|e^{-t A}\right\| \leq c_{0}$.Por exemplo, isto ocorre se $A \in \mathcal{A}, \mathcal{A}$ como em 1.2.13 [Pazy[11], página 30, teorema 7.7] mais a observação sobre a perturbação do resolvente comentada em 1.2.19. Definimos

1. $D_{A}(\alpha, \infty):=\left\{f \in X, \sup _{0<s \leq 1}\left(s^{-\alpha}\left\|\left(e^{-s A}-I\right) f\right\|\right)\right.$ finito $\}$.

2. $D_{A}(\alpha):=\left\{f \in X, t^{-\alpha}\left\|\left(e^{-t A}-I\right) f\right\| \rightarrow 0\right.$ se $\left.t \rightarrow 0+\right\}$.

Definição 1.4.2 Para os espaço $D_{A}(\alpha, \infty)$ e $D_{A}(\alpha)$, definimos a norma

$$
\|f\|_{D_{A}(\alpha, \infty)}:=\|f\|+\sup _{0<s \leq 1}\left\|s^{-\alpha}\left(e^{-s A}-I\right) f\right\| .
$$

Proposição 1.4.3 Suponha $A \in \mathcal{A}, \mathcal{A}$ como em 1.2.13 . Então

1.para $t>0$,

$$
\begin{aligned}
\sup _{0<s<\infty} & \left\|s^{-\alpha}\left(e^{-s A}-I\right)\left(e^{-t A}-I\right) f\right\| \\
\leq & \left(c_{0}+1\right) \sup _{0<s \leq t}\left(s^{-\alpha}\left\|\left(e^{-s A}-I\right) f\right\|\right) .
\end{aligned}
$$

2. $\left\|\left(e^{-t A}-I\right) f\right\|_{D_{A}(\alpha, \infty)} \leq\left\|\left(e^{-t A}-I\right) f\right\|+\left(c_{0}+1\right) \sup _{0<s \leq t}\left(s^{-\alpha}\left\|\left(e^{-s A}-I\right) f\right\|\right.$

Prova. Para ver 1 , dividimos a análise em dois casos. Por um lado,

$$
\begin{aligned}
& \sup _{0<s \leq t}\left\|s^{-\alpha}\left(e^{-s A}-I\right)\left(e^{-t A}-I\right) f\right\| \\
& \leq\left(c_{0}+1\right) \sup _{0<s \leq t}\left(s^{-\alpha}\left\|\left(e^{-s A}-I\right) f\right\|\right) .
\end{aligned}
$$


E por outro lado,

$$
\begin{gathered}
\sup _{t<s<\infty}\left\|s^{-\alpha}\left(e^{-s A}-I\right)\left(e^{-t A}-I\right) f\right\| \\
\leq\left(c_{0}+1\right) t^{-\alpha}\left\|\left(e^{-t A}-I\right) f\right\| .
\end{gathered}
$$

As duas estimativas juntas implicam 1 .

Com a definição de norma em $D_{A}(\alpha, \infty)$ e 1 , obtemos 2 .

Proposição 1.4.4 Suponha $A \in \mathcal{A}, \mathcal{A}$ como em 1.2.13. Então, uniformemente em $\mathcal{A}$,

1.para $t>0, c=1 / \alpha$ e $\alpha \in(0,1]$,

$$
\sup _{0<s \leq t}\left(s^{-\alpha}\left\|\left(e^{-s A}-I\right) f\right\|\right) \leq c \sup _{0<s \leq t}\left(s^{1-\alpha} \|\left(A e^{-s A} f \|\right)\right.
$$

2. para $c=c_{1} /\left(1-2^{\alpha-1}\right)$ e e $\alpha \in[0,1)$,

$$
\sup _{0<s<\infty}\left(s^{1-\alpha}\left\|A e^{-s A} f\right\|\right) \leq c \sup _{0<s<\infty}\left(s^{-\alpha}\left\|\left(e^{-s A}-I\right) f\right\|\right) .
$$

3. $\operatorname{para} c=c_{1} /\left(1-2^{\alpha-1}\right)$ e e $\alpha \in[0,1)$

$$
\sup _{0<s<\infty}\left(s^{1-\alpha}\left\|A e^{-s A} f\right\|\right) \leq c \sup _{0<s \leq 1}\left(s^{-\alpha}\left\|\left(e^{-s A}-I\right) f\right\|\right)+c\left(c_{0}+1\right)\|f\| .
$$

Prova. O item 1 é consequência direta do item 1 de 1.2.35.

A prova de 2 requer um argumento bem sofisticado. Pelo fato de $e^{-t A}$ ser semigrupo, a identidade seguinte é válida para todo natural $n$

$$
e^{-t A}=\sum_{j=0}^{n} e^{-2^{j} t A}\left(I-e^{-2^{j} t A}\right)+e^{-2^{n+1} t A} .
$$

Portanto, para $t>0$,

$$
\left\|A e^{-t A} f\right\| \leq \sum_{j=0}^{n}\left\|A e^{-2^{j} t A}\right\|\left\|\left(I-e^{-2^{j} t A}\right) f\right\|+\left\|A e^{-2^{n+1} t A} f\right\| .
$$

Suponha que $t>\epsilon>0$. Por 1.2.19,

$$
t^{1-\alpha}\left\|A e^{-t A} f\right\| \leq c_{1} \sum_{j=0}^{n}\left(2^{j}\right)^{\alpha-1} \sup _{\epsilon<s<\infty}\left(s^{-\alpha}\left\|\left(e^{-s A}-I\right) f\right\|+c_{1} / 2^{n+1} t^{-\alpha}\|f\| .\right.
$$


Quando $n \rightarrow \infty$,

$$
t^{1-\alpha}\left\|A e^{-t A} f\right\| \leq c_{1} /\left(1-2^{\alpha-1}\right) \sup _{\epsilon<s<\infty}\left(s^{-\alpha}\left\|\left(e^{-s A}-I\right) f\right\|\right.
$$

o que prova 2 , pois $\epsilon>0$ é arbitrário.

A prova de 3 é consequência do item 2 e da estimativa $\sup _{1<s<\infty}\left(s^{-\alpha}\left\|\left(e^{-s A}-I\right) f\right\| \leq\right.$ $c_{0}+1$ em consequência de 1.2.19.

Proposição 1.4.5 Para $A \in \mathcal{A}, \mathcal{A}$ como em 1.2.13, $\alpha \in(0,1), D_{A}(\alpha, \infty) \doteq$ $X_{\alpha, 1 ; \infty}^{\prime}(A)$.

Prova. Consequência direta de 1 e 3 de 1.4.4.

Proposição 1.4.6 Para $A \in \mathcal{A}, \mathcal{A}$ como em 1.2.13, $\alpha \in(0,1) D_{A}(\alpha) \doteq$ $X_{\alpha, 1 ; \infty}^{o}(A)$.

Prova. Por 1.2.37, $\operatorname{Lip}(\alpha, X, D(A)) \doteq X_{\alpha, 1 ; \infty}^{o}(A)$ se $\alpha \in(0,1)$. Mas, por um lado, o item 1 de 1.2.35 implica $\operatorname{Lip}(\alpha, X, D(A)) \subset D_{A}(\alpha)$. Por outro lado, pelo item 2 da proposição 1.4 .3 se $f \in D_{A}(\alpha)$,

$$
\left\|\left(e^{-t A}-I\right) f\right\|_{D_{A}(\alpha, \infty)} \rightarrow 0
$$

quando $t \rightarrow 0+\mathrm{e}$, pela proposição 1.4.5, $\left\|e^{-t A}-I\right\|_{X_{\alpha, 1 ; \infty}(A)} \rightarrow 0$ também quando $t \rightarrow 0+$. Isto justifica a imersão $D_{A}(\alpha) \subset X_{\alpha, 1 ; \infty}^{o}(A)$.

\subsection{Estimativas com o resolvente}

Esta seção contém estimativas para o resolvente $\lambda-(-A)$ em relação aos espaços intermediários $X_{\alpha, \mathbf{1} ; \infty}^{\prime}(A)$. No capítulo seguinte, elas vão ser um dos ingredientes para avaliar a dependência do semigrupo em $f, e^{-t A} f$, $f \in X_{\alpha, 1 ; \infty}^{\prime}(A)$, quando variamos $A$ em $\mathcal{A}$.

Teorema 1.5.1 Suponha $\mathcal{A}$ como em 1.2.13. Então se $A, B \in \mathcal{A}, \lambda \in$ $\rho(-A)$,

1. $\left\|(\lambda+A)^{-1}\right\|_{L\left(X, X_{\alpha, 1 ; \infty}^{\prime}(B)\right)} \leq c(|\lambda|+1)^{\alpha-1}$, com constante que nãa depende de $A, B$ e especificamente se $A=B, c=\frac{2^{1-\alpha}}{\Gamma(2-\alpha)}(\alpha)^{-\alpha} c_{0} C_{\mathcal{A}}^{1-\alpha}\left(C_{\mathcal{A}}+1\right)^{\alpha}$ 
2. $\left\|A(\lambda+A)^{-1}\right\|_{L\left(X_{\alpha, 1 ; \infty}^{\prime}(B), X\right)} \leq c|\lambda|^{-\alpha}$, com constante que não depende de $A, B$ e especificamente se $A=B, c=\Gamma(\alpha)\left(1+2 C_{\mathcal{A}}\right)$.

Prova. É possível provar uma desigualdade mais forte que 1. Suponha $f \in X$, vamos avaliar $\left\|A^{\alpha}(\lambda+A)^{-1} f\right\|$. Pela desigualdade de interpolação de [Henry[8], página 26, teorema 1.4.4], $\left\|A^{\alpha} x\right\| \leq c\|A x\|^{\alpha}\|x\|^{1-\alpha}$, com $c=$ $2^{1-\alpha} \alpha^{-\alpha} c_{0} / \Gamma(2-\alpha)$ se $x \in D(A)$. No nosso caso particular, com a definição 1.2.13,

$$
\left\|A^{\alpha}(\lambda+A)^{-1} f\right\| \leq c C_{\mathcal{A}}^{1-\alpha}\left(C_{\mathcal{A}}+1\right)^{\alpha}(|\lambda|+1)^{\alpha-1} .
$$

Pelo teorema 1.3.1, conclui-se 1.

Para provar 2, recorremos à descrição do resolvente em função do semigrupo [Butzer e Berens[3], página 32, teorema 1.3.5]

$$
(\lambda+A)^{-1} f=\int_{0}^{\infty} e^{-\lambda s} e^{-s A} f d s
$$

se $\mathbb{R} e \lambda>\omega_{0}=\lim _{t \rightarrow \infty}\left(t^{-1} \log \left\|e^{-t A}\right\|\right)$. Então $\omega_{0} \leq-\delta$ e, portanto, a igualdade vale se $\mathbb{R} e \lambda \geq 0$.

Portanto preparando também para usar a regularidade de $f$,

$$
A(\lambda+A)^{-1} f=\int_{0}^{\infty} e^{-\lambda s} s^{\alpha-1} s^{1-\alpha} A e^{-s A} f d s .
$$

Para $\lambda \in \rho(-A), \operatorname{com}|\lambda|>0$,

$$
\left\|A(|\lambda|+A)^{-1} f\right\| \leq \Gamma(\alpha) \sup _{0<s<\infty}\left(s^{1-\alpha}\left\|A e^{-s A} f\right\|\right)|\lambda|^{-\alpha}
$$

No caso geral,quando $\lambda \in \rho(-A)$ pela identidade do resolvente,

$$
(\lambda-(-A))^{-1}=(|\lambda|-\lambda)(|\lambda|-(-A))^{-1}(\lambda-(-A))^{-1}+(|\lambda|-(-A))^{-1},
$$

e mais uma vez pela identidade do resolvente: $A(\lambda+A)^{-1}=[1-(|\lambda|-\lambda)(\lambda+$ $\left.A)^{-1}\right] A(|\lambda|+A)^{-1}$, chega-se a

$$
\left\|A(\lambda+A)^{-1} f\right\| \leq\left(1+2 C_{\mathcal{A}}\right)\left\|A(|\lambda|+A)^{-1} f\right\| .
$$

A estimativa recai no caso $\mathbb{R e} \lambda \geq 0$, o que conclui a prova de 2 .

Observação 1.5.2 Cálculo direto mostra que $\left\|(\lambda+A)^{-1}\right\|_{L\left(X_{\alpha, 1, \infty}^{\prime}(A)\right)} \leq 2 C_{\mathcal{A}} /(|\lambda|+$ 1). Note que $e^{-t A}=\frac{1}{2 \pi 1} \int_{\Gamma} e^{\lambda t}(\lambda+A)^{-1} d \lambda$ para um certo contorno $\Gamma$ em $\rho(-A)$. Então $(\lambda+A)^{-1} e^{-t A}=e^{-t A}(\lambda+A)^{-1}$. O resto é analisar a definição de norma em $X_{\alpha, 1 ; \infty}^{\prime}(A)$. 


\section{Capítulo 2}

\section{Estimativas e analiticidade do Semigrupo}

O objetivo deste capítulo é fornecer estimativas para descrever como os semigrupos $e^{-h A} \operatorname{com}-A \in \mathcal{A}, \mathcal{A}$ como em 1.2 .13 , se relacionam com os espaços $X_{\alpha, 1 ; \infty}^{\prime}(A)$. A referência [Henry[8], seção 1.4, especificamente teorema 1.4.3] fonece um bom guia para os enunciados dos teoremas, mas há muitas diferenças nas demonstrações.

Proposição 2.0.3 Suponha $\alpha, \beta \in[0,1]$ Seja $A \in \mathcal{A}, \mathcal{A}$ como em 1.2.13. Suponha $T$ um operador linear que obedece

$$
\|T\|_{L\left(X_{\alpha, 1 ; \infty}\left(A_{0}\right), X_{\beta, 1 ; \infty}\left(A_{0}\right)\right)} \leq c
$$

para algum $A_{0} \in A$. então

1. $\|T\|_{L\left((X, D(A))_{\alpha, \infty ; K},(X, D(B))_{\beta, \infty ; K}\right)} \leq c^{\prime} c$

2. $\|T\|_{L\left(X_{\alpha, 1 ; \infty}(A), X_{\beta, 1 ; \infty}(B)\right)} \leq c^{\prime \prime} c$

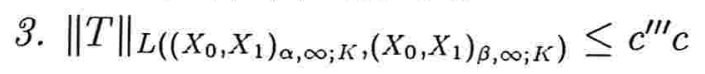

com $c^{\prime}, c^{\prime \prime}$ independentes de $A, B \in \mathcal{A}$.

Prova. É consequência direta da equivalência das normas descritas em 1.2.20 e 1.2.27.

Observação 2.0.4 A proposição anterior justifica um procedimento que vamos usar constantemente. Para a prova das estimativas com o semigrupo, escolhemos na definição dos espaços o próprio operador do semigrupo. A menos de uma constante, a estimativa valerá também para espaços com operadores distintos. 


\subsection{Estimativas para semigrupo}

Teorema 2.1.1 Suponha $A \in \mathcal{A}, \mathcal{A}$ como em 1.2.13. Então

1. Se $h \geq 0,0 \leq \beta \leq \alpha \leq 1$,

$$
\left\|e^{-h A}\right\|_{L\left(X_{\beta, 1 ; \infty}^{\prime}, X_{\alpha, 1 ; \infty}^{\prime}\right)} \leq c h^{\beta-\alpha} e^{-h \delta / 2} .
$$

Para espaços intermediários definidos com $A$, $c=c_{0} \max \left\{\left(2(\alpha-\beta) e^{-1} / \delta\right)^{\alpha-\beta}, 2^{1-\beta}\right\}$.

2.Se $h \geq 0,0 \leq \alpha \leq \beta \leq 1$,

$$
\left\|e^{-h A}\right\|_{L\left(X_{\beta, 1 ; \infty}^{\prime}, X_{\alpha, 1 ; \infty}^{\prime}\right)} \leq c e^{-h \delta / 4} .
$$

Para espaços definidos com o mesmo $A$, $c=c_{0} \max \left\{1,\left(4(\beta-\alpha) e^{-1} / \delta\right)^{\beta-\alpha}\right.$

Prova. Pela 2.0.3, nada impede de definirmos os espaços intermediários com o operador do semigrupo. Supondo $f \in X_{\beta, 1 ; \infty}(A)$,

$$
\left\|e^{-h A}\right\|_{\alpha, 1 ; \infty}=\left\|e^{-h A} f\right\|+\sup _{0<s<\infty}\left(s^{1-\alpha}\left\|A e^{-(s+h) A} f\right\|\right) .
$$

A parcela difícil de estimar é a segunda, pois, quanto a primeira, $\left\|e^{-h A} f\right\| \leq c_{0} \max _{h \geq 0}\left\{h^{\alpha-\beta} e^{-h / 2 \delta}\right\} e^{-h / 2 \delta} h^{\beta-\alpha}\|f\|$ no caso 1 e $\left\|e^{-h A} f\right\| \leq$ $c_{0} e^{-h \delta}$ no caso 2 .

Uma vez que $s^{1-\alpha} \leq(s+h)^{\beta-\alpha}(s+h)^{1-\beta}$, que implica, no caso 1 ,

$$
\begin{aligned}
& \sup _{0<s<\infty}\left(s^{1-\alpha}\left\|A e^{-(s+h) A}\right\|\right) \\
& \quad \leq 2^{1-\beta} c_{0} e^{-\delta h / 2} h^{\beta-\alpha} \sup _{0<s<\infty}\left(((s+h) / 2)^{1-\beta}\left\|A e^{-(s+h) / 2 A} f\right\|\right),
\end{aligned}
$$

concluindo 1 .

No caso 2 , a única diferença é que usamos $(s+h)^{\beta-\alpha} e^{-\delta(s+h) / 2} \leq$ $\sup _{0<s<\infty}\left\{s^{\beta-\alpha} e^{-\delta s / 4}\right\} e^{-\delta h / 4}$.

Observação 2.1.2 É possível adaptar a prova para obter uma estimativa com maior decaimento exponencial. Na verdade, qualquer decaimento $e^{-h \delta /(1+\mu)}$, qualquer $\mu>0$, é possível. A constante que entra no lugar do 2 acima é $(1+\mu) / \mu$. Esta observação se aplica para as demais estimativas com decaimento exponencial. Provavelemente, isto facilita a substituição dos espaços de potência por espaços intermdiários na teoria assintótica de [[8]], por exemplo, [[8], teorema 5.1.1]. 
Teorema 2.1.3 Suponha $A \in \mathcal{A}, \mathcal{A}$ como em 1.2.13. Então, uniformemente em $B, C \in \mathcal{A}$, para $h \geq 0$,

$$
\left\|e^{-h A}-I\right\|_{L\left(X_{\beta, 1 ; \infty}^{\prime}(B), X_{\alpha, 1 ; \infty}^{\prime}(C)\right)} \leq c \max \left\{h^{\beta-\alpha}, h^{\beta}\right\}
$$

quando $0 \leq \alpha \leq \beta \leq 1$. Além disso, $c=c_{0}+1$ se $\alpha=\beta$ e $c=\max \left\{c_{0}+\right.$ $\left.1, c_{1} / \beta, 1 / \beta\right\}$ quando $\beta>\alpha$.

Prova. Pela 2.0.3, é suficiente fazer a prova para $B=C=A$. Suponha $f \in$ $X_{\beta, 1 ; \infty}^{\prime}(A)$. Não há dúvida que $f \in X_{\alpha, 1 ; \infty}^{\prime}(A)$ pela 2.1.1 no caso particular $h=0$.

Para estimar a norma de $\left(e^{-h A}-I\right) f$ em $X_{\alpha, 1 ; \infty}^{\prime}(A)$,

$$
\left\|\left(e^{-h A}-I\right) f\right\|_{X_{\alpha, 1 ; \infty}^{\prime}(A)}=\left\|\left(e^{-h A}-I\right) f\right\|+\sup _{0<s<\infty}\left(s^{1-\alpha}\left\|A e^{-s A}\left(e^{-h A}-I\right) f\right\|\right),
$$

o único trabalho é em relação à segunda parcela. Para a primeira, a estimativa está em por 1.2.25 quando $\beta>0$.

Primeiramente, suponha $\beta=\alpha$, incluindo, portanto, $\alpha=0$. Neste caso,

$$
\left\|e^{-h A}-I\right\|_{L\left(X_{\alpha, 1 ; \infty}^{\prime}(A), X_{\alpha, 1 ; \infty}^{\prime}(A)\right.} \leq\left(c_{0}+1\right),
$$

com a retirada de $\left(e^{-h A}-I\right)$, que comuta com $A e^{-s A}$ e tem norma em $L(X)$ menor que $c_{0}+1$ pela 1.2.19.

Quando $\beta>\alpha$, por um lado se $s \leq h$, então $s^{\beta-\alpha} \leq h^{\beta-\alpha}$ e

$$
\left\|s^{1-\alpha} A e^{-s A}\left(e^{-h A}-I\right) f\right\| \leq\left(c_{0}+1\right) h^{\beta-\alpha}\left\|s^{1-\beta} A e^{-s A} f\right\| .
$$

Por outro lado, se $s>h, s^{-\alpha}<h^{-\alpha}$, e, portanto, $s^{1-\alpha}\left\|A e^{-s A}\right\| \leq c_{1} h^{-\alpha}$, o que justifica a desigualdade

$$
\left\|s^{1-\alpha} A e^{-s A}\left(e^{-s A}-I\right) f\right\| \leq c_{1} h^{\beta-\alpha}\left\|h^{-\beta}\left(e^{-h A}-I\right) f\right\| .
$$

Então, pelo item 1 da 1.2.35,

$$
\left\|s^{1-\alpha} A e^{-s A}\left(e^{-s A}-I\right) f\right\| \leq \frac{c_{1}}{\beta} h^{\beta-\alpha} \sup _{0<s \leq h}\left(s^{1-\beta}\left\|A e^{-s A} f\right\|\right) .
$$

Em ambos os casos,

$$
\begin{aligned}
&\left\|s^{1-\alpha} A e^{-s A}\left(e^{-h A}-I\right) f\right\| \leq c h^{\beta-\alpha} \sup _{0<s<\infty}\left(s^{1-\beta}\left\|A e^{-s A} f\right\|\right), \\
& \operatorname{com} c=\max \left\{c_{0}+1, c_{1} / \beta\right\} .
\end{aligned}
$$


Teorema 2.1.4 Suponha $A \in \mathcal{A}, \mathcal{A}$ como em 1.2.13. Então

1. se $\beta \in[0,1]$ e $B \in \mathcal{A}$,

$$
\left\|A e^{-h A}\right\|_{L\left(X_{\beta, 1 ; \infty}^{\prime}(B), X\right)} \leq c e^{-\delta h / 2} h^{\beta-1} .
$$

com constante $c$ uniforme em $\mathcal{A}$. Se $B=A$ então $c=2^{1-\beta} c_{0}$.

2. quando $\alpha, \beta \in[0,1]$ e $B, C \in \mathcal{A}$,

$$
\left\|A e^{-h A}\right\|_{L\left(X_{\beta, 1 ; \infty}^{\prime}(B), X_{\alpha, 1 ; \infty}^{\prime}(C)\right)} \leq c e^{-\delta h / 2} \max \left\{h^{\beta-1}, h^{\beta-\alpha-1}\right\} .
$$

com contante $c$ uniforme em $\mathcal{A}$. Além diso, se $B=A=C$, então $c=$ $\max \left\{2^{2-\beta} c_{1}, 2^{1-\beta} c_{0}\right\}$.

3. quando $\beta \in[0,1]$ e $\alpha \geq 1$,

$$
\left.\left\|A^{\alpha} e^{-h A}\right\|_{L\left(X_{\beta, 1, \infty}^{\prime}(A), X_{0}\right.}\right) \leq c(\alpha, \beta) e^{-\delta h / 2} h^{\beta-\alpha} .
$$

Prova. Computação direta que leva à norma em $X_{\beta, 1 ; \infty}(A)$ produz a desigualdade 1 .

Suponha $f \in X_{\beta, 1 ; \infty}^{\prime}(A)$. Pela proposição 1.2 .19

$$
\left\|A e^{-h A} f\right\| \leq c_{0} e^{-\delta h / 2}\left\|A e^{-h A / 2} f\right\|
$$

Com o ajuste pela substituição $1=2^{1-\beta} h^{\beta-1}(h / 2)^{1-\beta}$,

$$
\left\|A e^{-h A} f\right\| \leq 2^{1-\beta} c_{0} e^{-\delta h / 2} h^{\beta-1} \sup _{0<s<\infty}\left(s^{1-\beta}\left\|A e^{-s A} f\right\|\right),
$$

o que conclui a prova.

Para obter 2, suponha novamente $f \in X_{\beta, 1 ; \infty}(A)$. Por definição,

$$
\left\|A e^{-h A} f\right\|_{X_{\alpha, 1 ; \infty}^{\prime}(A)}=\left\|A e^{-h A} f\right\|+\sup _{0<s<\infty}\left(s^{1-\alpha}\left\|A e^{-s A} A e^{-h A} f\right\|,\right.
$$

evidenciando a necessidade de avaliar somente a segunda parcela, pois a primeira parcela está avaliada no item 1.

Pela propriedade de semigrupo, $A e^{-s A} A e^{-h A}=A^{2} e^{-(s+h) A}=$ $A e^{-(s+h) A / 2} A e^{-(s+h) A / 2}$ e, portanto,

$$
\left\|A e^{-s A} A e^{-h A}\right\| \leq 2 c_{1} e^{-\delta h / 2}(s+h)^{-1}\left\|A e^{-(s+h) A / 2}\right\|,
$$


onde também foi aplicada 1.2 .19 e a desigualdade óbvia $e^{-\delta(s+h) / 2} \leq e^{-\delta h / 2}$ se $s>0$.

Resta somente reorganizar a desigualdade prévia para fazer surgir a norma de $f$ em $X_{\beta, 1 ; \infty}^{\prime}(A)$. Portanto substituímos $1=2^{1-\beta}(s+h)^{\beta-1}((s+h) / 2)^{1-\beta}$ e então, como $s^{1-\alpha}(s+h)^{-1} \leq(s+h)^{-\alpha}$ e $(s+h)^{\beta-\alpha-1} \leq h^{\beta-\alpha-1}$,

$$
s^{1-\alpha}\left\|A e^{-s A} A e^{-h A}\right\| \leq 2^{2-\beta} c_{1} e^{-\delta h / 2} h^{\beta-\alpha-1} \sup _{0<s<\infty}\left(s^{1-\beta}\left\|A e^{-s A} f\right\|\right),
$$

de onde se pode concluir a prova. A terceira estimativa utiliza a estimativa com potência fracionária.

$$
\left\|A^{\alpha} e^{-h A}\right\|_{L\left(X_{0}\right)} \leq c(\alpha) h^{-\alpha} e^{-\delta h},
$$

$\alpha \geq 0$, pela [Henry[8], página 26, teorema 1.4.3]. Note que $c(\alpha)$ é constante em $\mathcal{A}$. Sobolevskii também fornece uma tal estimativa, mas restringe desnecessariamente o valor de $\alpha$. Para concluir, estime

$$
\left\|A^{\alpha} e^{-h A}\right\|_{L\left(X_{\beta}, X_{0}\right)} \leq\left\|A^{\alpha-1} e^{-h A / 2}\right\|_{L\left(X_{0}\right)}\left\|A e^{-h A / 2}\right\|_{L\left(X_{\beta}, X_{0}\right)}
$$

com aplicação também da estimativa do item 1.

\subsection{Analiticidade do semigrupo}

Para provar a analiticidade de $(\xi, A) \rightarrow e^{-t A} \xi$, é necessário antes definir uma topologia para os operadores $A$ onde o semigrupo esteja localmente bem definido. O candidato provável é o conjunto $\mathcal{H}\left(X_{1}, X_{0}\right)$ da definição 1.2.13.

Proposição 2.2.1 O conjunto $\mathcal{H}\left(X_{1}, X_{0}\right)$ é um subconjunto aberto de $L\left(X_{1}, X_{0}\right)$. $E$ cada $A \in \mathcal{H}\left(X_{1}, X_{0}\right)$ possui uma vizinhança aberta em $\mathcal{H}\left(X_{1}, X_{0}\right)$ que também é um conjunto regular de $\mathcal{H}\left(X_{1}, X_{0}\right)$.

Prova. Obviamente é necessário provar que $\mathcal{H}\left(X_{1}, X_{0}\right) \subset L\left(X_{1}, X_{0}\right)$. Para ver isto, tomando qualquer $A_{0} \in \mathcal{H}\left(X_{1}, X_{0}\right)$, defina $a_{1}$ a constante de imersão para $D\left(A_{0}\right) \subset X_{1}$ e por $a_{2}$ a constante de imersão para $X_{1} \subset D\left(A_{0}\right)$. Então se $f \in X_{1}=D\left(A_{0}\right),\|f\|_{X_{0}}+\left\|A_{0} f\right\|_{X_{0}} \leq a_{2}\|f\|_{X_{1}}$ e, portanto, $\left\|A_{0} f\right\|_{X_{0}} \leq$ $a_{2}\|f\|_{X_{1}}$ e $A_{0} \in L\left(X_{1}, X_{0}\right)$.

Seja $M_{0}$ tal que $(|\lambda|+1)\left\|(\lambda+A)^{-1}\right\|_{L\left(X_{0}\right)} \leq M_{0}$ para $\mathbb{R e} \lambda \geq 0$.

Concluir a prova significa achar $B\left(A_{0}, r\right)$, uma bola de centro $A_{0}$ e raio $r$ de $L\left(X_{1}, X_{0}\right)$ que está contida em $\mathcal{A}$. 
Chame de $P$ um elemento de $B\left(A_{0}, r\right)$. Sempre é verdade que $(\lambda+P)=$ $\left[I+\left(P-A_{0}\right)\left(\lambda+A_{0}\right)^{-1}\right]\left(\lambda+A_{0}\right)$. E também que $\left(P-A_{0}\right)\left(\lambda+A_{0}\right)^{-1}=$ $\left(P-A_{0}\right) A_{0}^{-1} A_{0}\left(\lambda+A_{0}\right)^{-1}$.

Por um lado

$$
A_{0}\left(\lambda+A_{0}\right)^{-1}=-\lambda\left(\lambda+A_{0}\right)^{-1}+I,
$$

o que implica $\left\|A_{0}\left(\lambda+A_{0}\right)^{-1}\right\|_{L\left(X_{0}\right)} \leq M_{0}+1$.

E por outro lado, como $\left\|A_{0}^{-1}\right\|_{L\left(X_{0}, X_{1}\right)} \leq a_{1}\left(M_{0}+1\right)$,

$$
\left\|\left(P-A_{0}\right) A_{0}^{-1}\right\| \leq\left\|P-A_{0}\right\|_{L\left(X_{1}, X_{0}\right)}\left\|A_{0}^{-1}\right\|_{L\left(X_{0}, X_{1}\right)} \leq a_{1}\left(M_{0}+1\right) r .
$$

Com isto se $r \leq\left(a_{1}\left(M_{0}+1\right)^{2}\right)^{-1} / 2$, teremos $\lambda+P$ é inversível e

$$
\left\|(\lambda+P)^{-1}\right\|_{L\left(X_{0}\right)} \leq\left\|\left(\lambda+A_{0}\right)^{-1}\right\|_{L\left(X_{0}\right)}\left\|\sum_{k=0}^{\infty}(-1)^{k}\left(P-A_{0}\right)\left(\lambda+A_{0}\right)^{-1}\right\|_{L\left(X_{0}\right)},
$$

e então $\left\|(\lambda+P)^{-1}\right\|_{L\left(X_{0}\right.} \leq 2 M_{0} /(|\lambda|+1)$.

Falta provar a equivalência das normas do gráfico de $D(P)$ e $X_{1}$. Para isto, é suficiente demonstrar que $A_{0} P^{-1}$ e $P A_{0}^{-1}$ são uniformemente limitados em uma bola $B\left(A_{0}, r\right)$, talvez diminuindo $r$. Suponha $f \in X_{1}=D\left(A_{0}\right)=D(P)$. Por um lado,

$$
\|f\|_{X_{1}} \leq a_{1}\left(\|f\|+\left\|A_{0} P^{-1}\right\|_{L\left(X_{0}\right)}\|P f\|_{X_{0}}\right),
$$

então $\|f\|_{X_{1}} \leq a_{1} \max \left\{1,\left\|P A_{0}^{-1}\right\|_{L\left(X_{0}\right)}\right\}\left(\|f\|_{X_{0}}+\|P f\|_{X_{0}}\right)$. Por outro lado, $\|f\|_{X_{0}}+\|P f\|_{X_{0}} \leq a_{2} \max \left\{1,\left\|P A_{0}^{-1}\right\|_{L\left(X_{0}\right)}\right\}\|f\|_{X_{1}}$.

O $r$ que escolhemos antes também garante que $r<\left(a_{1}\left(M_{0}+1\right)\right)^{-1} / 2$, então $\left(P-A_{0}\right) A_{0}^{-1}$ tem norma em $L\left(X_{0}\right)$ menor que $1 / 2$ e, portanto, pela igualdade

$$
A_{0} P^{-1}=\left[I+\left(P-A_{0}\right) A_{0}^{-1}\right]^{-1},
$$

obtemos $\left\|A P_{0}^{-1}\right\|_{L\left(X_{0}\right)}<2$.

O cáculo para avaliar a norma de $P A_{0}^{-1}$ em $L\left(X_{0}\right)$ é bem mais simples, pois

$$
P A_{0}^{-1}=\left(P-A_{0}\right) A_{0}^{-1}+I,
$$

que leva a $\left\|P A_{0}^{-1}\right\|_{L\left(X_{0}\right.} \leq a_{1}\left(M_{0}+1\right)+1$.

Observação 2.2.2 Integrabilidade em espaços de Banach. Seja $B_{\gamma}((0, T), X)$ com $X$ espaço de Banach definida como as funçôes em contínuas de $(0, T)$ 
em $X$ com $g(s) s^{\gamma}$ limitado em $(0, T)$ e cujo sup é a norma do espaço. Se $g \in B_{\gamma} C((0, T), X)$ e $\gamma<1$ então $\int_{0}^{t} g d s$ existe e pertence à $C([0, T], X) . O$ argumento consiste em uma aplicação do Teorema da Convergência Dominada de Lebesgue, por exemplo, como está em [Dunford e Schwartz[9], volume 1, página 151, teorema 16]. Tome $g_{n}$ definida por g em $[1 / n, T-1 / n]$ e por seus valores em $1 / n$ e $T-1 / n$ para tornar $g_{n}$ contínua em $[0, T]$ e, portanto, elemento de $L^{1}((0, T), X)$. Então $\left\|g_{n}\right\|_{X} \leq\|g\|_{X} \leq c_{1} t^{-\gamma}$. Tome $x_{0} \in X$ com norma 1 e chame $D(t)=c_{1} t^{-\gamma} x_{0}$. Então $g_{n} \rightarrow g$ em $X$ pontualmente em $[0, T]$.Também $g_{n}$ é limitada ou dominada por $D$ em $[0, T]$. Como $\left.D \in L^{1}(0, T), X\right)$ então, pelo Teorema da Convergência Dominada de Lebesgue, $g$ também pertence e $g_{n} \rightarrow g$ em $L^{1}((0, T), X)$. Para concluir, note que $\left\|\int_{t_{0}}^{t} g(s) d s\right\|$ é menor que $\left|t^{1-\gamma}-t_{0}^{1-\gamma}\right| /(1-\gamma)$.

Teorema 2.2.3 Suponha $\mathcal{A}$ como em 1.2.13, $0<T<\infty, 0 \leq \beta \leq \alpha \leq 1$ e chame $\left(X_{0}, X_{1}\right)_{\alpha, \infty ; K}^{o}:=X_{\alpha}$. Então

$$
(A, \xi) \rightarrow\left\{e^{-t A} \xi, 0 \leq t \leq T\right\}: \mathcal{H}\left(X_{1}, X_{0}\right) \times X_{\alpha} \rightarrow C\left([0, T], X_{\alpha}\right)
$$

e

$$
(A, g) \rightarrow\left\{\int_{0}^{t} e^{-(t-s) A} g(s) d s, 0 \leq t \leq T\right\}: \mathcal{H}\left(X_{1}, X_{0}\right) \times C\left([0, T], X_{\beta}\right) \rightarrow C\left([0, T], X_{\alpha}\right)
$$

são ambas analíticas.

Prova. Ambas funções estão bem definida. Como

$$
\left\|e^{-t_{2} A} \xi-e^{-t_{1} A} \xi\right\|_{L\left(X_{\alpha}\right)} \leq\left\|e^{-m A}\right\|_{L\left(X_{\alpha}\right)}\left\|\left(e^{-\left|t_{2}-t_{1}\right| A}-I\right) \xi\right\|,
$$

com $m=\min \left\{t_{1}, t_{2}\right\}$, usando 2.1.1 e o fato que o semigrupo é fortemente contínuo em $X_{\alpha}$, então $\left\|e^{-t_{2} A} \xi-e^{-t_{1} A} \xi\right\|_{L\left(X_{\alpha}\right)} \rightarrow 0$ se $\left|t_{2}-t_{1}\right| \rightarrow 0$ e então $t \rightarrow e^{-t A} \xi \in C\left([0, T], X_{\alpha}\right)$. Para analisar o outro caso, suponha $t_{2} \geq t_{1}$. Pela propriedade de semigrupos,

$$
\begin{aligned}
\int_{0}^{t_{2}} & e^{-t_{2} A} g(s) d s-\int_{0}^{t_{1}} e^{-t_{1} A} g(s) d s \\
& =\int_{t_{1}}^{t_{2}} e^{-\left(t_{2}-s\right) A} g(s) d s+\int_{0}^{t_{1}}\left(e^{-\left(t_{2}-t_{1}\right) A}-I\right) e^{-\left(t_{1}-s\right) A} g(s) d s .
\end{aligned}
$$

Avaliar a primeira parcela não oferece dificuldade, pois, pela 2.1.1, $\left\|\int_{t_{1}}^{t_{2}} e^{-\left(t_{2}-s\right)} g(s) d s\right\|_{X_{\alpha}} \leq$ $c\left(t_{2}-t_{1}\right)^{1+\beta-\alpha}\|g\|_{C\left([0, T], X_{\beta}\right)}$. Para a avaliar a outra parcela, seja $\delta, 0<\delta<$ 


$$
\begin{aligned}
& 1+\beta-\alpha \text { e faça } \\
& ]\left\|\int_{0}^{t_{1}}\left(e^{-\left(t_{2}-t_{1}\right) A}-I\right) e^{-\left(t_{1}-s\right) A} g(s) d s\right\|_{X_{\alpha}} \\
& \quad \leq \int_{0}^{t_{1}}\left\|e^{-\left(t_{2}-t_{1}\right) A}-I\right\|_{L\left(X_{\alpha+\delta}, X_{\alpha}\right)}\left\|e^{-\left(t_{1}-s\right) A}\right\|_{L\left(X_{\beta}, X_{\alpha+\delta}\right)} d s\|g\|_{C\left([0, T], X_{\beta}\right)} .
\end{aligned}
$$

Então usando 2.1 .1 e 2.1.3

$$
\left\|\int_{0}^{t_{1}}\left(e^{-\left(t_{2}-t_{1}\right) A}-I\right) e^{-\left(t_{1}-s\right) A} g(s) d s\right\|_{X_{\alpha}} \leq c\left(t_{2}-t_{1}\right)^{\delta} t_{1}^{1+\beta-\alpha-\delta} /(1+\beta-\alpha-\delta),
$$

que completa a justificativa que $t \rightarrow \int_{0}^{t} e^{-s A} g(s) d s$ pertence à $C\left([0, T], X_{\alpha}\right)$.

Também vê-se que em relação à $\xi$ e à $g$ ambas funções são lineares e contínuas. Então é suficiente provar a analiticidade somente em relação à $A$.

Para $\|H\|_{L\left(X_{1}, X_{0}\right)}$ pequeno o que equivale a $\left\|H A^{-1}\right\|$ pequeno em $L(X)$, $A+H \in \mathcal{A}$ e então

$$
e^{-s(A+H)} \xi-e^{-s A} \xi=-\frac{1}{2 \pi i} \int_{\Gamma} e^{s \lambda}(\lambda+A+H)^{-1} H A^{-1}\left[A(\lambda+A)^{-1}\right] \xi d \lambda
$$

se $\left.\Gamma=\Gamma_{-} \cup \Gamma_{0} \cup \Gamma_{+} \operatorname{com} \Gamma_{0}=\left\{e^{i \Psi},|\Psi| \leq \phi\right\}, \Gamma_{ \pm}=\left\{\rho e^{ \pm i \phi} i\right\}, \rho \geq 1\right\} \mathrm{e}$ $\Gamma \pm$ tendo o sentido ascendente como orientação positiva e $\Gamma_{0}$ tendo o sentido anti-horário como orientação positiva; está sendo admitido $\phi \in(\pi / 2, \pi)$ cuja garantia da existência foi explicada na proposição 1.2.19.

A igualdade acima se justifica pelo fato de que $e^{-t A}$ e $e^{-t(A+H)}$ serem semigrupos analíticos e porque

$$
(\lambda+A+H)^{-1}-(\lambda+A)^{-1}=-(\lambda+A+H)^{-1} H(\lambda+A)^{-1} .
$$

Para $\left\|H A^{-1}\right\|<\left(C_{\mathcal{A}}+1\right)^{-1} / 2$,

$$
(\lambda+A+H)^{-1}=(\lambda+A)^{-1} \sum_{k=0}^{\infty}\left[H(\lambda+A)^{-1}\right]^{k},
$$

pois $\lambda+A+H=\left[I+H(\lambda+A)^{-1}\right](\lambda+A)$ e $H A^{-1} A(\lambda+A)^{-1}$ tem norma em $L(X)$ menor que $1 / 2$.

Portanto obtemos uma série de termos $k+1$ lineares e limitados em $H$

$e^{-s(A+H)} \xi-e^{-s A} \xi=-\frac{1}{2 \pi i} \int_{\Gamma} e^{s \lambda}(\lambda+A)^{-1} \sum_{k=0}^{\infty}\left[H(\lambda+A)^{-1}\right]^{k} H A^{-1}\left[A(\lambda+A)^{-1}\right] \xi d \lambda$. 
Mudando de variável $\lambda=s \mu$ e, pelo teorema de Cauchy, quando $s>0$, $e^{-s(A+H)} \xi-e^{-s A} \xi=-s^{-1} \frac{1}{2 \pi i} \int_{\Gamma} e^{\mu}(\mu / s+A)^{-1} \sum_{k=0}^{\infty}\left[H(\lambda+A)^{-1}\right]^{k} H A^{-1}\left[A(\mu / s+A)^{-1}\right] \xi d \mu$.

Então a norma em $X_{\alpha}$ de $\left(e^{-s(A+H)} \xi-e^{-s A} \xi\right)$ não excede

$$
c \int_{\Gamma}\left|e^{\mu}\right| \sum_{k=0}^{k=1}\left[\left\|H A^{-1}\right\|\left(C_{\mathcal{A}}+1\right)\right]^{k}\left\|H A^{-1}\right\||\mu|^{\alpha-1}|\mu|^{-\alpha}\|\xi\|_{X_{\alpha}},
$$

onde usamos o teorema 1.5.1 que também explicita $c$. Está série é uniformemente convergente em $[0, T]$ se a norma de $H A^{-1}$ em $L\left(X_{0}\right)$ é menor que $\left(C_{\mathcal{A}}+1\right)^{-1} / 2$ e seu valor é menor que $\left\|H A^{-1}\right\|(2 \pi)^{-1} \int_{\Gamma}\left|e^{\mu}\right||\mu|^{-1}|d \mu|$. Estamos antecipando o caso $s=0$ onde a série vale zero como explicado adiante.

Resta provar se as parcelas da série pertencem à $C\left([0, T], X_{\alpha}\right)$.

É suficiente provar que

$$
\int_{\Gamma}\left|e^{s \lambda}-e^{s_{0} \lambda}\left\|\left.\lambda\right|^{\alpha-1}\right\| A(\lambda+A)^{-1} \xi \|\right.
$$

tende a zero se $s \rightarrow s_{0}$ com $0 \leq s_{0}, s \leq T$.

No caso $0<\delta \leq s_{0}, s \leq T$, qualquer $0<\delta<T$, a continuidade é uniforme, pois

$$
\left|e^{s \lambda}-e^{s_{0} \lambda}\right| \leq\left|e^{\delta \lambda}\right|,
$$

pelo teorema do valor médio e pela estimativa $\left|e^{s \lambda}\right| \leq\left|e^{\delta \lambda}\right|$ se $s>\delta$, obtemos

$$
\begin{gathered}
\int_{\Gamma}\left|e^{s \lambda}-e^{s_{0} \lambda}\right||\lambda|^{-1}\left\|A(\lambda+A)^{-1} \xi\right\||d \lambda| \\
\leq \int_{\Gamma}\left|e^{\delta \lambda}\right||d \lambda|\|\xi\|_{X_{\alpha}}\left|s-s_{0}\right| .
\end{gathered}
$$

O caso $s_{0}=0$ é bem mais díficil.

Para um $f \in D(A)$, e quando $s=0$ na série, chamando

$$
F(\lambda)=(\lambda+A)^{-1} \sum_{k=0}^{\infty}\left[H(\lambda+A)^{-1}\right]^{k} H A^{-1}\left[A(\lambda+A)^{-1}\right] f,
$$

e se $\Gamma_{R}=B(0, R) \cap \Gamma, B(0, R)$ a bola centrada na origem de raio $R$, obtemos, pelo teorema de Cauchy,

$$
\int_{\Gamma_{R}} F(\lambda) d \lambda=\int_{\mathcal{C}_{R}} F(\lambda) d \lambda
$$


com $\mathcal{C}_{R}=\|\left\{R e^{i \Psi},,|\Psi| \leq \phi\right\}$. Fazendo $R \rightarrow \infty$, e notando que $\|F\|_{X_{\alpha}}=$ $O\left(|\lambda|^{\alpha-2}\right.$ ) então $\int_{\Gamma} F(\lambda) d \lambda$ vale zero. Para $s>0$, mudando de variável $\lambda=s \mu$, pelo teorema de Cauchy,

$$
\int_{\Gamma} e^{s \lambda} F(\lambda) d \lambda=s^{-1} \int_{\Gamma} e^{\mu} F(\mu / s) d \mu .
$$

Então como $\left\|A(\mu / s+A)^{-1} f\right\| \leq\left(C_{\mathcal{A}}+1\right)(\mu / s)^{-1}\|A f\|$,

$$
\left\|\int_{\Gamma} e^{s \lambda} F(\lambda) d \lambda\right\|_{X_{\alpha}} \leq 2 c s^{1-\alpha} \int_{\Gamma}\left|e^{\mu}\right||\mu|^{\alpha-2}|d \mu|\left\|H A^{-1}\right\|\|A f\|,
$$

que revela portanto que $\left\|\int_{\Gamma} e^{s \lambda} F(\lambda) d \lambda\right\|_{X_{\alpha}}$ tende a zero se $s \rightarrow 0+$ e $f \in$ $D(A)$.

Pela fato de que a série pertencere a $L\left(X_{\alpha}\right)$ e porque $D(A)$ é denso em $X_{\alpha}$, então o limite acima também dá zero se colocarmos $\xi$ no lugar de $f$.

Seja agora uma $p_{n}$ o polinômio

$$
\begin{aligned}
& p_{n}\left(H_{1}, \cdots, H_{n}\right) \\
& \quad=1 / n ! \sum_{\pi(1), \cdots, \pi(n)}\left\{-\frac{1}{2 \pi i} \int_{\Gamma} e^{s \lambda}(\lambda+A)^{-1}\left[H_{\pi(1)}(\lambda+A)^{-1}\right] \cdots\right. \\
& \left.\quad\left[H_{\pi(n-1)}(\lambda+A)^{-1}\right] H_{\pi(n)} A^{-1}\left[A(\lambda+A)^{-1}\right] \xi d \lambda\right\} .
\end{aligned}
$$

Pelo que vimos $p_{n}$ é uma polinomial em $L\left(L^{k}\left(X_{1}, X_{0}\right), C\left([0, T], X_{\alpha}\right)\right)$ que, por construção, é também simétrica e

$$
e^{-s(A+H)} \xi-e^{-s A} \xi=\sum_{k=1}^{\infty} p_{k}\left(H^{k}\right)
$$

com $H^{k}=H_{1}, \cdots, H_{k}$, uniformemente numa vizinhança de cada $A \in \mathcal{A}$. Portanto $A \rightarrow e^{-(\cdot) A} \xi$ é analítica de $\mathcal{A}$ em $C\left([0, t], X_{\alpha}\right)$, concluindo a prova do item 1.

Aproveitanto a expansão prévia,

$$
\left(e^{-s(A+H)}-e^{-s A}\right) g(s)=-\frac{1}{2 \pi i} \int_{\Gamma} e^{s \lambda}(\lambda+A)^{-1} \sum_{k=0}^{\infty}\left[H(\lambda+A)^{-1}\right]^{k} H A^{-1}\left[A(\lambda+A)^{-1}\right] g(s) d \lambda .
$$

Mudando de variável $s \lambda=\mu$, pelo teorema de Cauchy,

$$
\begin{aligned}
& \left(e^{-s(A+H)}-e^{-s A}\right) g(s)= \\
& \quad-\frac{1}{2 \pi i} s^{-1} \int_{\Gamma} e^{\mu}\left(\frac{\mu}{s}+A\right)^{-1} \sum_{k=0}^{\infty}\left[H\left(\frac{\mu}{s}+A\right)^{-1}\right]^{k} H A^{-1}\left[A\left(\frac{\mu}{s}+A\right)^{-1}\right] g(s) d \mu,
\end{aligned}
$$


ainda com a mesma $\Gamma$.

Então a norma em $X_{\alpha}$ desta série não excede

$$
c s^{\beta-\alpha} \int_{\Gamma}\left|e^{\mu}\right| \sum_{k=0}^{k=1}\left(\left\|H A^{-1}\right\|\left(C_{\mathcal{A}}+1\right)\right)^{k}\left\|H A^{-1}\right\|(|\mu|+1)^{\alpha-1}|\mu|^{-\beta}\|g(s)\|_{X_{\beta}},
$$

portanto tomando aqui também $\left\|H A^{-1}\right\|<\left(C_{\mathcal{A}}+1\right)^{-1} / 2$, e integrando em $s$,

$$
\begin{aligned}
& \left\|\int_{0}^{t}\left(e^{-s(A+H)}-e^{-s A}\right) g(s) d s\right\|_{C\left([0, T], X_{\alpha}\right)} \leq \\
& \quad c \frac{t^{1+\beta-\alpha}}{1+\beta-\alpha}\left\|H A^{-1}\right\|\|g\|_{C\left([0, T], X_{\beta}\right)} \int_{\Gamma}\left|e^{\mu}\right||\mu|^{\alpha-\beta-1}|d \mu|,
\end{aligned}
$$

sendo a série uniformemente convergente na vizinhança de $A$ com $\left\|H A^{-1}\right\| \leq$ $\left(C_{\mathcal{A}}+1\right)^{-1} / 2$. $T$,

Cada termo da série acima pertence à $C\left([0, T], X_{\alpha}\right)$, pois, com $0 \leq t_{1}, t_{2} \leq$

$$
\begin{aligned}
& \left\|\int_{t_{1}}^{t_{2}} s^{-1} \int_{\Gamma} e^{\mu}\left(\frac{\mu}{s}+A\right)^{-1}\left[H\left(\frac{\mu}{s}+A\right)^{-1}\right]^{k} H A^{-1}\left[A\left(\frac{\mu}{s}+A\right)^{-1}\right] g(s) d \mu\right\|_{X_{\alpha}} \\
& \leq c\left|t_{1}^{1+\beta-\alpha}-t_{2}^{1+\beta-\alpha}\right| /(1+\beta-\alpha)\left\|H A^{-1}\right\|\|g\|_{C\left([0, T], X_{\alpha}\right)} .
\end{aligned}
$$

Defina

$$
\begin{aligned}
& p_{n}\left(H_{1}, H \cdots, H_{n}\right) \\
& =1 / n ! \sum_{\pi(1), \cdots, \pi(n)}-\frac{1}{2 \pi i} \int_{0}^{t} s^{-1} \int_{\Gamma} e^{\mu}\left(\frac{\mu}{s}+A\right)^{-1}\left[H_{\pi(1)}\left(\frac{\mu}{s}+A\right)^{-1}\right] \\
& \quad \cdots\left[H_{\pi(2)}\left(\frac{\mu}{s}+A\right)^{-1}\right] H_{\pi(n)} A^{-1}\left[A\left(\frac{\mu}{s}+A\right)^{-1}\right] g(s) d \mu d s .
\end{aligned}
$$

Como visto $p_{n} \in L\left(L^{k}\left(X_{1}, X_{0}\right), C\left([0, T], X_{\alpha}\right)\right)$, são simétricos por construção e para cada $A \in \mathcal{A}, \sum_{k=0}^{\infty} p_{k}\left(H^{k}\right)=\int_{0}^{t}\left(e^{-s(A+H)}-e^{-s A}\right) g(s) d s$ em uma vizinhança de $A$. Então $\mathcal{A} \ni A \rightarrow \int_{0}^{t} e^{-t A} g(s) d s \in C\left([0, T], X_{\alpha}\right)$ é analítica.

Lema 2.2.4 Seja $\Gamma=\Gamma_{-} \cup \Gamma_{0} \cup \Gamma_{+} \operatorname{com} \Gamma_{0}=\left\{e^{i \Psi},|\Psi| \leq \phi\right\}, \Gamma_{ \pm}=$ $\left\{\rho e^{ \pm i \phi}, \rho \geq 1\right\}$ e $\Gamma \pm$ tendo o sentido ascendente como orientação positiva $e$ $\Gamma_{0}$ tendo o sentido anti-horário como orientação positiva. Então, se $\theta \geq 0$,

$$
\int_{\Gamma}\left|e^{\mu}\right||\mu|^{-\theta}|d \mu| \leq 2 \phi e-2 e^{\cos \phi} / \cos \phi
$$


50CAPÍTULO 2. ESTIMATIVAS E ANALITICIDADE DO SEMIGRUPO

Prova. Em $\Gamma_{0},|\mu|=1,\left|e^{\mu}\right|=e^{\cos \psi},|d \mu|=d \psi$. Então $\int_{\Gamma_{0}}\left|e^{\mu}\right||\mu|^{\theta}|d \mu|=$ $\int_{-\phi}^{\phi} e^{\cos \psi} d \psi \leq 2 e \phi$.

Quanto ao sentido do percurso, para $\Gamma_{-}, \rho:-\infty \rightarrow 1$ e, para $\Gamma_{+}$, $\rho: 1 \rightarrow+\infty$. Também em $\Gamma_{ \pm},|\mu|=\rho,|d \mu|= \pm d \rho,\left|e^{\mu}\right|=e^{\rho \cos \phi}$. Em ambos os casos, portanto,

$$
\int_{\Gamma_{ \pm}}\left|e^{\mu}\right| \mu|| d \mu \mid=\int_{1}^{\infty} e^{\rho \cos \phi} / \rho^{\theta} d \rho \leq-e^{\cos \phi} / \cos \phi .
$$




\section{Capítulo 3}

\section{Estimativas e analiticidade do Operador de Evolução}

É um fato bem estabelecido que a solução da equação em um espaço de Banach $X_{0}, d u(t) / d t+A(t) u(t)=0,0 \leq s \leq t \leq T, u(s)=x$ pode ser descrita através do chamado Operador de Evolução $U, u(t)=U(t, s) x$, quando o operador $A(t)$ é do tipo hiperbólico, tipo parabólico, etc. Há diversas referências para o fato com as hipóteses precisas necessárias, como [2], [1], [11], etc.

Neste capítulo vamos ver como o operador de evolução, quando $A(t)$ é do tipo parabólico, se comporta diante dos espaços $\left(X_{0}, X_{1}\right)_{\alpha, \infty ; K}$ supondo $D\left(A(t) \doteq X_{1}\right.$ uniformente com $t \in[0, T]$.

Originalmente, Sobolevskii já descreveu estimativas, digamos, do mesmo tipo, mas em relação às potências fracionárias do operador $A(t)$. Conforme discutido na observação 1.2.23, os espaços $\left(X_{0}, X_{1}\right)_{\alpha, \infty ; K}$ tem grande vantagem sobre os espaços de potência porque simplesmente não dependem do operador $A(t)$ e as estimativas com o operador de evolução valem, como veremos, uniformemente em uma família de operadores $A(t)$.

É difícil descrever as diferenças entre o que há neste capítulo e o que fizeram autores que também avaliaram os operadores de evolução nos espaços $\left(X_{0}, X_{1}\right)_{\alpha, \infty ; K}$, incluindo, entre eles, o professor Herbert Amann no seu livro [5]. No comentário que segue estou pensando no trabalho do professor Amann em relação ao que há de comum entre o que temos aqui e o que há em Sobolevskii, ou seja, admitindo que o domínio dos operadores $A(t)$ é constante com $t$. Comparativamente, para não errar muito, uma análise cuidadosa de nosso trabalho conclui que estamos mais próximos de Sobolevskii 
que de Amann, pois procuramos obter estimativas do operador específicas em relação aos espaços $\left(X_{0}, X_{1}\right)_{\alpha, \infty ; K}$ como fez Sobolevskii em relação aos espaços de potência de $A(t)$ enquanto Amann propõe estimativas do operador evolução não apenas para estes espaços, mas também para outros espaços de interpolação. Os argumentos são bem diferentes porque a igualdade entre $\left(X_{0}, X_{1}\right)_{\alpha, \infty ; K}$ e $X_{\alpha, 1 ; \infty}^{\prime}(A)$ quando $X_{0}=X$ e $D(A(t)) \doteq X_{1}$ é pouco explorada nos trabalhos de Amann e, no nosso caso, esta igualdade é muito utilizada. Nossas estimativas também são mais precisas, no sentido de que fornecemos estimativas para a continuidade o operador de evolução $U_{A}$ em relação a $t, s$ nos espaços intermediários remetendo bastante a Sobolevskii. Amann, por outro lado, para ganhar em generalidade, procura sempre fornecer estimativas para vários espaços de interpolação simultaneamente, o que provavelmente traz limitações.

Para comodidade do leitor, antes de passar para as estimativas, a seção seguinte contém os resultados principais que vamos admitir sobre operadores de evolução para operadores do tipo parabólico.

Também está incluída uma descrição de uma desigualdade de Gronwall, ingrediente utilizado em muitas das estimativas que vamos fazer, não só aqui, mas em outras partes. Neste capítulo, a desigualdade de Gronwall fornece a primeira estimativa que é um dos pontos de partida para as demais. Para tentar algum controle das constantes que aparecem nas estimativas, vamos descrever a constante da desigualdade de Gronwall como uma função dos parâmetros para mais fácil referência.

\subsection{Operador de evolução do tipo parabólico e desigualdade de Gronwall}

Definição 3.1.1 Sejam $X_{0}$ e $X_{1}$ espaços de Banach com $X_{1} \subset X_{0}$ contínua e densamente. Seja $\mathcal{H}\left(X_{1}, X_{0}\right)$ o conjunto definido em 1.2.13, $T>0$ e $\epsilon \in(0,1]$. Definimos

$$
\begin{aligned}
C^{\epsilon}\left([0, T], \mathcal{H}\left(X_{1}, X_{0}\right)\right)= & \\
& \left\{A, A:[0, T] \rightarrow \mathcal{H}\left(X_{1}, X_{0}\right), \text { sua imagem } R(A)\right. \text { é um } \\
& \text { conjunto regular de } \mathcal{H}\left(X_{1}, X_{0}\right) ; \\
& \text { existe uma constante, } c>0, c=c(A), \text { tal que } \\
& \left.\left\|(A(t)-A(s)) A^{-1}(s)\right\| \leq c|t-s|^{\epsilon} \text { quando } t, s \in[0, T]\right\} .
\end{aligned}
$$




\subsection{OPERADOR DE EVOLUÇÃO DO TIPO PARABÓLICO E DESIGUALDADE DE GRONW.}

Um conjunto regular de $C^{\epsilon}\left([0, T], \mathcal{H}\left(X_{1}, X_{0}\right)\right)$, denotado por $\mathcal{A} \subset C^{\epsilon}\left([0, T], \mathcal{H}\left(X_{1}, X_{0}\right)\right.$, é um conjunto tal que $\{A(t), t \in[0, T], A \in \mathcal{A}\}$ é um conjunto regular de $\mathcal{H}\left(X_{1}, X_{0}\right)$ e existe uma constante $a>0$ para que $\left\|(A(t)-A(s)) A^{-1}(s)\right\| \leq$ $a|t-s|^{\epsilon}$ para todo $A \in \mathcal{A} \subset C^{\epsilon}\left([0, T], \mathcal{H}\left(X_{1}, X_{0}\right)\right)$.

Equivalentemente, um conjunto regular de $C^{\epsilon}\left([0, T], \mathcal{H}\left(X_{1}, X_{0}\right)\right)$, denotado por $\mathcal{A} \subset C^{\epsilon}\left([0, T], \mathcal{H}\left(X_{1}, X_{0}\right)\right)$, é um conjunto cujos elementos obedecem às seguintes condições

1. $\left\|(\lambda+A(t))^{-1}\right\|_{L\left(X_{0}\right)} \leq \frac{C_{\mathcal{A}}}{|\lambda|+1}$ com constante $C_{\mathcal{A}}$ não dependente de $A \in \mathcal{A}$, $t \in[0, T]$ e $\lambda \in\{\operatorname{Re} \lambda \geq 0\}$.

2. $D(A(t)) \doteq X_{1}$ com constantes de imersão que não dependem de $A(t)$ para $A \in \mathcal{A}$ e $t \in[0, T]$.

3. Existe uma constante a e um número $\epsilon, \epsilon \in(0,1]$, para que

$$
\left\|(A(t)-A(s)) A(\tau)^{-1}\right\| \leq a|t-s|^{\epsilon},
$$

uniformente com $A \in \mathcal{A}$ e $t, s, \tau \in[0, T]$.

Observação 3.1.2 Sabendo que $D(A(t)) \doteq X_{1}$ para algum $A \in \mathcal{A}$ e $t \in$ $[0, T]$, a hipótese 3 implica 2 .

Observação 3.1.3 Na definição de $C^{\epsilon}\left([0, T], \mathcal{H}\left(X_{1}, X_{0}\right)\right)$ quando, como é o caso aqui, $[0, T]$ é um conjunto compacto, a hipótese de a imagem de $A$ ser um conjunto regular de $\mathcal{H}\left(X_{1}, X_{0}\right)$ é consequência automática da continuidade de $t \rightarrow A(t):[0, T] \rightarrow L\left(X_{1}, X_{0}\right)$, portanto pode ser removida. $A$ demonstração será omitida. Entretanto, isto não ocorreria em casos mais gerais, por exemplo, com $[0, \infty)$ no lugar de $[0, T]$.

Teorema 3.1.4 Suponha $A \in C^{\epsilon}\left([0, T], \mathcal{H}\left(X_{1}, X_{0}\right)\right)$ conforme 3.1.1. Então existe um operador de evolução $U_{A}(t, s)$ em $0 \leq s \leq t \leq T$ que obedece

1. $U_{A}(t, s)=U_{A}(t, \tau) U_{A}(\tau, s)$ para $0 \leq s \leq \tau \leq t \leq T$ e $U_{A}(t, t)=I$ para $t \in[0, T]$.

2. para $0 \leq s \leq t \leq T, U_{A}(t, s) \in L\left(X_{0}\right)$ e é fortemente contínuo, ou seja, $U_{A}(t, s) x$ é contínua na norma de $X_{0}$ para cada $x \in X_{0}$. E para $0 \leq s<$ $t \leq T, U_{A}(t, s) X_{0} \subset X_{1}$ e é uniformemente diferenciável com $t$, ou seja, 
$\partial U_{A}(t, s) / \partial t \in L\left(X_{0}\right)$ existe na norma de $L\left(X_{0}\right)$; além disto, esta derivada é fortemente contínua e obedece a equação em $0 \leq s<t \leq T$

$$
\frac{\partial U_{A}(t, s)}{\partial t}+A(t) U_{A}(t, s)=0 .
$$

3. à equação em $[s, T], 0 \leq \tau \leq t \leq T$,

$$
U_{A}(t, \tau)=e^{-(t-\tau) A(\tau)}+\int_{\tau}^{t} U_{A}(t, s)[A(\tau)-A(s)] e^{-(s-\tau) A(\tau)} d s .
$$

4. à equação em $[\tau, T], 0 \leq \tau \leq t \leq T$,

$$
U_{A}(t, \tau)=e^{-(t-\tau) A(t)}+\int_{\tau}^{t} e^{-(t-s) A(t)}[A(t)-A(s)] U_{A}(s, \tau) d s .
$$

Prova. As afirmações 1 e 2 foram retiradas de [Soboleviskii[1], página 4, teorema 1] e de [Pazy[11], página 150, teorema 6.1] Então as afirmações valem para todo $A \in \mathcal{A}$. A afirmação 3 foi retirada de [Sobolevskii[1], página 8], sendo de resto através dela que Sobolevskii defini o operador de evolução $U_{A}(t, \tau)$. Finalmente, a afirmação 4 é fornecida em [Soboleviskii[1], página 20, item 8] ou [Amann[5], página 48].

Recomendamos a pesquisa destes resultados também em Kato [10] e em Tanabe [2].

Observação 3.1.5 Alessandra Lunardi [[16], capítulo 6] constrói um operador de evolução para um par de espaços $X_{1} \subset X_{0}$ com imersão contínua, mas sem exigir densidade de $X_{1}$ em $X_{0}$. Entretanto, não há garantia de continuidade com $t, s$ se $0 \leq s \leq t \leq T$, mas somente se $0 \leq s<t \leq T$. Neste caso, o operador também é diferenciável em $L\left(X_{0}\right)$, ou seja, uniformemente diferenciável [[16], página 211, definição 6.0.1].

Teorema 3.1.6 Suponha $A \in C^{\epsilon}\left([0, T], \mathcal{H}\left(X_{1}, X_{0}\right)\right)$ conforme 3.1.1. Então a equação em u em $X_{0}$, para $x \in X_{0}, 0 \leq \tau<t \leq T$,

$$
\frac{d u(t)}{d t}+A(t) u(t)=f(t), u(\tau)=x
$$

possui

1. se $f \in L^{1}\left(\tau, T ; X_{0}\right)$, uma única solução mild

$$
u(t)=U_{A}(t, \tau) x+\int_{\tau}^{t} U_{A}(t, s) f(s) d s,
$$

ou seja, está garantido somente que $[\tau, t] \rightarrow u(t) \in X_{0}$ é contínua. 


\subsection{OPERADOR DE EVOLUÇÃO DO TIPO PARABÓLICO E DESIGUALDADE DE GRONW}

2. se $f$ é Hölder contínua de $[0, T]$ em $X_{0}$, uma solução clásica, ou seja, $u(t) \in X_{1}$ em $(\tau, T]$ e é também continuamente diferenciável de $(\tau, T] \mathrm{em}$ $X_{0}$. A equação é verificada na norma de $X_{0}$ e sua solução também pode ser descrita pela fórmula do item 1.

Prova. Para o item 1, [Pazy[11], página 168]. Para o item 2, [Sobolevskii[1], página 4, teorema 4] ou [Pazy[11], página 168, teorema 7.1].

Teorema 3.1.7 Suponha $a \geq 0, b \geq 0,0 \leq \alpha, \beta<1$ e $u:[0, T] \rightarrow \mathbb{R}$ integrável

$$
0 \leq u(t) \leq a t^{-\alpha}+b \int_{0}^{t}(t-s)^{-\beta} u(s) d s
$$

q.t.p em $0<t \leq T$. Então existe uma constante $c_{G}=c_{G}(a, b, \alpha, \beta)$ para que

$$
u(t) \leq c_{G} t^{-\alpha},
$$

q.t.p em $(0, T]$. A constante $c_{G}$ depende dos parâmetros de acordo com

$$
c_{G}=c(\beta, b) e^{\theta T} a /(1-\alpha), \theta=(b(-\beta) !)^{1 /(1-\beta)},
$$

segundo [Henry[8], página 189] e [Henry[?], página 104] ou

$$
c_{G}=a\left(1+c(\alpha, \beta, \epsilon) b t^{1-\beta} e^{(1+\epsilon) \mu(\beta, b) t}\right), \mu(\beta, b)=(\Gamma(1-\beta) b)^{1 /(1-\beta)},
$$

segundo[Amann[5], página 52, teorema 3.3.1] . Para referência, denotamos $c_{G}=c_{G}(a, b, \alpha, \beta)$.

Prova. Contida nas referência citadas no enunciado.

Observação 3.1.8 Não vou me ater ao problema de explicitar a constante de Gronwall. Não foram mencionadas todas as hipóteses do caso de Amann porque acho que sua prova está defeituosa, então recomendo referência original. A desigualdade de Gronwall tem papel relevante na análise do comportamento assintótico em equações de evolução. Uma boa contribuição daria quem fizesse o [Henry[8], página 190, exercício 3] explicitando o comportamento da constante. Também talvez descobrir o efeito de se incluir um exponencial como em $u(t) \leq a t^{\alpha-1}+b \int_{0}^{t} e^{-\delta(t-s)}(t-s)^{\beta-\alpha} s^{\gamma-1} d s$. 


\subsection{Ferramentas do cálculo em espaço de Ba- nach}

Teoremas do cálculo em espaço de Banach para os quais não achei uma referência explícita, provavemente devido à sua especifidade, são colocados nesta seção. Embora possam ser considerados simples, no sentido de que teoremas neste assunto nos forçam a retirar todas as idéias desnecessárias, demonstram ser ferrramentas poderosíssimas no nosso trabalho.

Também, para comodidade do leitor, acrescentei os teoremas gerais sobre o assunto que vão ser usados aqui.

\section{Resultados gerais}

Definição 3.2.1 Suponha $X, Y$ espaços de Banach complexos e $U \subset X$, um aberto. Uma função $f: U \subset X \rightarrow Y$ é analítica em $U$ se para todo $x_{0} \in U$ existe $\delta\left(x_{0}\right)>0$ tal que se $\|h\|_{X}<\delta, x_{0}+h \in X$ então

$$
f\left(x_{0}+h\right)=\sum_{n=0}^{\infty} p_{n}\left(h^{n}\right)
$$

com $p_{n} \in L_{\text {sym }}^{n}(X, Y) e\left(h^{n}\right)=(h, \cdots, h)$.

Observação 3.2.2 Esta definição incluindo a condição de uniformidade da convergência da série em uma vizinhança de cada $x_{0}$ garantida pelo teorema abaixo é usada em [Henry[8], página 11]. Exatamente com o presente texto é usada em [Lunardi[16], página 513] e, segundo citação aí, em [Prodi e Ambrosetti[18]]. A definição aqui proposta é conveniente para nós porque reduz a prova da analiticidade a achar os polinômios que compõem a série. Aqui, pelo menos, a prova da convergência da série sempre é obtida provando diretamentente a convergência uniforme da série em um vizinhança de cada $x_{0}$.

Teorema 3.2.3 Suponha $X, Y$ espaços de Banach e $U \subset X$, aberto. Então se $f$ é analítica em $U, f$ é $C^{\infty}$ em $U$ e, com a notação da definição 3.2.1, $f^{(n)}\left(x_{0}\right)=n ! p_{n}, n \geq 0$. Também existe um $\delta_{1}\left(x_{0}\right)>0$ para que $f(x+h)=$ $\sum_{n=0}^{\infty} \frac{1}{n !} f^{(n)}(x)\left(h^{n}\right)$ uniformemente em $x, h$ se $\|h\|_{X}+\left\|x-x_{0}\right\|_{X}<\delta_{1}$.

Prova. [Henry[13], capítulo analytic function in complex Banach spaces, página (2.40)] e, para última afirmação, [Henry[13], capítulo analytic function 
in complex Banach spaces, página (2.36)] ou [Hale[12], página 23, teorema 1.10].

Teorema 3.2.4 Suponha $f$ analítica como na definição 3.2.1. Então para cada $x_{0} \in U$, existe um $\delta_{1}\left(x_{0}\right)>0$ e $M\left(x_{0}, \delta_{1}\right)$ para que $\left\|p_{n}\right\|_{L_{s y m}^{n}(X, Y)} \delta_{1}^{n} \leq$ $M<\infty, n \geq 0$.

Prova. [Henry[13], capítulo analytic function in complex Banach spaces, página (2.40)] e [Henry[8],página 11].

Observação 3.2.5 Em consequência, é possível achar $\bar{\delta}$ para $\left\|p_{n}\right\|_{L_{s y m}^{n}(X, Y)} \bar{\delta}^{n} \leq$ $M \theta^{n}, n \geq 0,0<\theta<1$. Para ver isto, tome $\bar{\delta}=\delta_{1} \theta$.

Teorema 3.2.6 Sejam $U$ aberto em $X$ com $X, Y$ espaços de Banach complexos. Suponha

(i) $f_{n}: U \rightarrow Y$ analítica para $n \geq 1$.

(ii) $\left\|f_{n}(x)\right\|_{Y} \leq C<\infty$ para todo $x \in A$ e $n \geq 1$.

(iii) $\lim _{n \rightarrow \infty} f_{n}(x)=f(x)$ existe e a convergência é uniforme para $x \in U$, ou seja, $\sup _{x \in U}\left|f_{n}(x)-f(x)\right| \rightarrow 0$, quando $n \rightarrow \infty$.

Então $f: U \rightarrow Y$ é analítica, $\|f(x)\| \leq C$ para $x \in U$ e, se $U_{1} \subset U$ tem $B_{\delta}\left(U_{1}\right) \subset$ A para algum $\left.\delta>0, f_{n}^{(} k\right) \rightarrow f^{(k)}(x)$ quando $n \rightarrow \infty$ uniformemente para $x \in U_{1}$, para cada $k \geq 0$. Especificamente $\sup _{x \in U_{1}}\left\|f_{n}^{(k)}(\cdot)-f^{(k)}(\cdot)\right\|_{L_{s y m}^{k}(X, Y)} \leq$ $k ! \delta^{-k} \sup _{U}\left\|f_{n}-f\right\|$.

Prova. [Henry[13], página (2.39)] ou, com um enunciado menos detalhado, [Lunardi[16], página 515, corolário 4.10].

\section{Resultados específicos}

Definição 3.2.7 Suponha $X, Y$ espaços de Banach e $0<T<\infty$. Seja $\alpha$ um número real e seja $\Delta=\{(t, s), 0 \leq s<t \leq T\}$. Chamamos de $B_{\alpha} C(\Delta, L(X, Y))$ o conjunto das funções $f$ em $C(\Delta, L(X, Y))$ e que possuem o valor

$$
\|f\|_{B_{\alpha} C(\Delta, L(X, Y))}:=\sup _{(t, s) \in \Delta}(t-s)^{\alpha}\|f(t, s)\|_{L(X, Y)}
$$

finito e escolhido para norma do espaço. 
Observação 3.2.8 O espaço $B_{\alpha} C(\triangle, L(X, Y))$ é um espaço de Banach [Amann [5], página 48]. Modifiquei a notação original de Amann que o chamava de $\mathcal{K}_{\infty}(E, F, \alpha)$ em que lá o sup era tomado em $\left\{(t, s) \in J_{\Delta}, s<t\right\}$ com $J_{\Delta}:=\{(t, s) \in J \times J ; s \leq t\}$. Este conjunto coincide com $\Delta$ nosso se $J=[0, T]$.

Proposição 3.2.9 Suponha $X, Y, Z$ espaços de Banach. Então se $a \in$ $B_{\alpha} C(\Delta, L(Y, Z)) e b \in B_{\beta} C(\Delta, L(X, Y))$, a função $a b,(a b)(t, s)=a(t, s) b(t, s)$, pertence à $B_{\alpha+\beta} C(\Delta, L(X, Z))$ e

$$
\|a b\|_{B_{\alpha+\beta} C(\Delta, L(X, Z))} \leq\|a\|_{B_{\alpha} C(\Delta, L(Y, Z))}\|b\|_{B_{\beta} C(\Delta, L(X, Y))} .
$$

Prova. Seja $K$ um compacto de $\mathbb{R}^{2}$ contido em $\Delta$. Obviamente $a$ e $b$ são uniformemente contínuas e limitadas em $K$. Sejam então $m_{b}$ e $m_{b}$ para $\|a\|_{C(K, L(Y, Z))} \leq m_{a}$ e $\|b\|_{C(K, L(X, Y))} \leq m_{b}$. Portanto, para $\left(t_{2}, s_{2}\right)$ e $\left.t_{1}, s_{1}\right)$ em $K$, como $a\left(t_{2}, s_{2}\right)-a\left(t_{1}, s_{1}\right)=\left(a\left(t_{2}, s_{2}\right)-a\left(t_{1}, s_{1}\right)\right) b\left(t_{2}, s_{2}\right)+a\left(t_{1}, s_{1}\right)\left(b\left(t_{2}, s_{2}\right)-\right.$ $\left.b\left(t_{1}, s_{1}\right)\right)$,

$$
\begin{aligned}
& \left\|a\left(t_{2}, s_{2}\right)-a\left(t_{1}, s_{1}\right)\right\|_{L(X, Z)} \\
& \quad \leq m_{b}\left\|a\left(t_{2}, s_{2}\right)-a\left(t_{1}, s_{1}\right)\right\|_{L(Y, Z)}+m_{a}\left\|b\left(t_{2}, s_{2}\right)-b\left(t_{1}, s_{1}\right)\right\|_{L(X, Y)},
\end{aligned}
$$

o que implica que também $a b$ é uniformente contínua em $K$. Mas como $K$ é arbitrário em $\Delta, a b \in C(\Delta, L(X, Z))$.

A última afirmação é consequência direta da aplicação da definição de norma em $B_{\alpha+\beta} C(\Delta, L(X, Z))$.

Definição 3.2.10 O símbolo $f * g$ denota a convolução de duas funções $f, g$, $t, s \rightarrow f(t, s), g(t, s)$, e é definida por $(f * g)(t, s)=\int_{s}^{t} f(t, \tau) g(\tau, s) d \tau$.

Proposição 3.2.11 Suponha $X, Y, Z$ espaços de Banach. Então se $a \in$ $B_{\alpha} C(\Delta, L(Y, Z))$ e $b \in B_{\beta} C(\Delta, L(X, Y)), \alpha, \beta \in(-\infty, 1)$, então $a * b \in B_{\alpha+\beta-1} C(\triangle, L(X, Z)) e$ $\|a * b\|_{B_{\alpha+\beta-1} C(\Delta, L(X, Z))} \leq B(1-\alpha, 1-\beta)\|a\|_{B_{\alpha} C(\Delta, L(Y, Z))}\|b\|_{B_{\beta} C(\Delta, L(X, Y))}$.

Prova. A parte mais difícil consiste em verificar que $a * b \in C(\Delta, L(X, Z))$.

Suponha $0 \leq s_{0}<t_{0}<t_{1} \leq T$. Em particular, é necessário provar que, na norma de $L(X, Z), a * b\left(t_{1}, s_{0}\right)-a\left(t_{0}, s_{0}\right) \rightarrow 0$ se $t_{1} \rightarrow t_{0}$. Os demais casos seguem o mesmo tipo de argumento. 
Seja $\delta>0$. Seu valor vai ser definido abaixo, mas já admita $\delta<\left(t_{0}-\right.$ $\left.s_{0}\right) / 2$. Então

$$
\begin{aligned}
& a * b\left(t_{1}, s_{0}\right)-a * b\left(t_{0}, s_{0}\right)= \\
& \quad \int_{s_{0}}^{t_{0}-\delta}\left(a\left(t_{1}, \tau\right)-a\left(t_{0}, \tau\right)\right) b\left(\tau, s_{0}\right) d \tau \\
& \quad+\int_{t_{0}-\delta}^{t_{0}} a\left(t_{1}, \tau\right) b\left(\tau, s_{0}\right) d \tau-\int_{t_{0}-\delta}^{t_{0}} a\left(t_{0}, \tau\right) b\left(\tau, s_{0}\right) d \tau+\int_{t_{0}}^{t_{1}} a\left(t_{1}, \tau\right) b\left(\tau, s_{0}\right) d \tau .
\end{aligned}
$$

A parcela que não depende de $\delta$ tem norma em $L(X, Z)$ que não excede $c \int_{t_{0}}^{t_{1}}\left(t_{1}-\tau\right)^{-\alpha}\left(\tau-s_{0}\right)^{-\beta} d \tau \operatorname{com} c=\|a\|_{B_{\alpha} C(\Delta, L(Y, Z))}\|b\|_{B_{\beta} C(\Delta, L(X, Y))}$. Portanto menor que $\epsilon / 4$ se $\left|t_{1}-t_{0}\right|<\delta_{1}:=\left\{\epsilon\left[c \max \left\{\left(t_{0}-s_{0}\right)^{-\beta}, T^{-\beta}\right\} /(1-\right.\right.$ $\left.\alpha)]^{-1} / 4\right\}^{1 /(1-\alpha)}$.

Como $\left\|a\left(t_{1}, \tau\right)\right\|_{L(Y, Z)} \leq\left(t_{1}-\tau\right)^{-\alpha}\|a\|_{B_{\alpha} C(\Delta, L(Y, Z))}$ e $\left\|b\left(\tau, s_{0}\right)\right\|_{L(X, Y)} \leq$ $(\tau-s)^{-\beta}\|b\|_{B_{\beta} C(\Delta, L(X, Y))}$ então

$$
\begin{aligned}
& \left\|\int_{t_{0}-\delta}^{t_{0}} a\left(t_{1}, \tau\right) b\left(\tau, s_{0}\right) d \tau\right\|_{L(X, Z)} \\
& \quad \leq \max \left\{2^{\beta}, 1\right\}\left(t_{0}-s_{0}\right)^{-\beta}\left[\left(t_{1}-t_{0}+\delta\right)^{1-\alpha}-\left(t_{1}-t_{0}\right)^{1-\alpha}\right] /(1-\alpha) .
\end{aligned}
$$

Escolha $\delta, \delta_{2}$, com $\delta<\left(t_{0}-s_{0}\right) / 2$ e $\delta_{2}<\delta_{1}$, para que o último majorante seja menor que $\epsilon / 4$.

Seja também que $\delta$ seja pequeno o suficiente para

$$
\left\|\int_{t_{0}-\delta}^{t_{0}} a\left(t_{0}, \tau\right) b\left(\tau, s_{0}\right) d \tau\right\|_{L(X, Z)} \leq \delta^{1-\alpha} /(1-\alpha)<\epsilon / 4 .
$$

Seja $\delta$ conveniente para as majorações prévias fixado.

Como $(t, s) \rightarrow a(t, s)$ é uniformemente contínua em $U=\left\{(t, s), s_{0} \leq s \leq\right.$ $\left.t_{0}-\delta<t_{0} \leq t \leq T\right\}$ então existe $\bar{\delta}>0, \bar{\delta}<\delta_{2}$, para que $\left\|a(t, s)-a\left(t^{\prime}, s^{\prime}\right)\right\|_{L(Y, Z)}<$ $c^{\prime} \in / 4$, com $c^{\prime}=\frac{\left(t_{0}-s_{0}\right)^{1-\beta}}{1-\beta}\|b\|_{B_{\beta}(\Delta, L(X, Y))}$ quando $(t, s),\left(t^{\prime}, s^{\prime}\right) \in U$. Isto implica que

$$
\left\|\int_{s_{0}}^{t_{0}-\delta}\left(a\left(t_{1}, \tau\right)-a\left(t_{0}, \tau\right)\right) b\left(\tau, s_{0}\right) d \tau\right\|_{L(X, Z)} \leq \epsilon / 4
$$

que completa a justificativa de que $a * b \in C(\Delta, L(X, Y))$.

Para completa a prova é suficiente notar que

$$
\begin{aligned}
& \|a * b(t, s)\|_{L(X, Z)} \\
& \quad \leq c B(1-\alpha, 1-\beta)(t-s)^{1-\alpha-\beta},
\end{aligned}
$$


$\operatorname{com} c=\|a\|_{B_{\alpha} C(\Delta, L(Y, Z))}\|b\|_{B_{\beta} C(\Delta, L(X, Y))}$.

Para análise dos demais casos, é conveniente notar que, pela forma do domínio $\Delta$, é suficiente demonstrar a continuidade de $a * b(t, s)$ separadamente em $t$ e $s$.

Teorema 3.2.12 Suponha $X, Y, Z, W$ espaços de Banach, $U \subset X$ um aberto e $\alpha, \beta \in(-\infty, 1)$. Se

$$
x \rightarrow a(x): U \rightarrow B_{\alpha} C(\triangle, L(Z, W))
$$

$e$

$$
x \rightarrow b(x): U \rightarrow B_{\beta} C(\triangle, L(Y, Z))
$$

são funções analíticas então, se $(a * b)(x)=a(x) * b(x)$,

$$
x \rightarrow(a * b)(x): U \rightarrow B_{\alpha+\beta-1} C(\Delta, L(Y, W))
$$

é analítica.

E explitamente se $a\left(x_{0}+h\right)=\sum_{n=0}^{\infty} p_{a}^{n}\left(h^{n}\right)$ e $b\left(x_{0}+h\right)=\sum_{n=0}^{\infty} p_{b}^{n}\left(h^{n}\right)$, sendo $p_{a}^{n} \in L_{\text {sym }}^{n}\left(X, B_{\alpha} C(\Delta, L(Z, W))\right.$ e $p_{b}^{n} \in L_{\text {sym }}^{n}\left(X, B_{\beta} C(\Delta, L(Y, Z))\right.$, então

$$
(a * b)\left(x_{0}+h\right)=\sum_{n=0}^{\infty} q^{n}\left(h^{n}\right)
$$

com

$$
q^{n}\left(h^{n}\right)=\sum_{0 \leq r \leq n} p_{a}^{r}\left(h^{r}\right) * p_{b}^{n-r}\left(h^{n-r}\right) .
$$

Prova. Por hipótese, para cada $x_{0} \in U$, existe $\delta\left(x_{0}\right)$ para

$$
a\left(x_{0}+h\right)=\sum_{n=0}^{\infty} p_{a}^{n}\left(h^{n}\right)
$$

e

$$
b\left(x_{0}+h\right)=\sum_{n=0}^{\infty} p_{b}^{n}\left(h^{n}\right)
$$

se $\|h\|_{X}<\delta\left(x_{0}\right)$, sendo $p_{a}^{n} \in L_{\text {sym }}^{n}\left(X, B_{\alpha} C(\Delta, L(Z, W))\right.$ e $p_{b}^{n} \in L_{s y m}^{n}\left(X, B_{\beta} C(\Delta, L(Y, Z))\right.$. Também é válido que, talvez diminuindo um pouco o $\delta$, pelo teorema 3.2.4, $\delta^{n}\left\|p_{a}^{n}\right\|_{L^{n}\left(X, B_{\alpha}(\Delta, L(Z, W))\right)} \leq M_{a}<\infty$ e $\delta^{n}\left\|p_{b}^{n}\right\|_{L^{n}\left(X, B_{\beta}(\Delta, L(Y, W))\right)} \leq M_{b}<\infty$. Admita, sem perda de geralidade, que $M_{a}$ e $M_{b}$ foram escolhidos para que também $M_{a} M_{b} B(1-\alpha, 1-\beta) \geq 1$. 


\subsection{FERRAMENTAS DO CÁLCULO EM ESPAÇO DE BANACH}

Pela proposição 3.2.11, $a\left(x_{0}+h\right) * b\left(x_{0}+h\right) \in B_{\alpha+\beta-1} C(\triangle, L(Y, W))$.

Defina

$$
q^{n}\left(h_{1}, \cdots, h_{n}\right):=\sum_{0 \leq r \leq n, \pi} \frac{p_{a}^{r}\left(h_{1}, \cdots, h_{r}\right) * p_{b}^{n-r}\left(h_{r+1}, \cdots, h_{n}\right)}{C_{n, r}},
$$

fazendo somente as permutações $\pi$ entre os índices de $\{1, \cdots, r\}$ e $\{r+$ $1, \cdots, n\}$.

Então, também com a aplicação da proposição 3.2.11, e esta série é convergente quando $\|h\|_{X}<\delta /\left(2 B(1-\alpha, 1-\beta) M_{a} M_{b}\right)$, pois, se $n \geq 1$, $\left\|q^{n}\left(h^{n}\right)\right\|_{B_{\alpha+\beta-1} C(\Delta, L(Y, W))}$

$$
\leq\|h\|_{X}^{n} B(1-\alpha, 1-\beta)\left\|p_{a}^{r}\right\|_{L_{s y m}^{r}\left(X, B_{\alpha}(\Delta, L(Z, W))\right)}\left\|p_{b}^{n-r}\right\|_{L_{s y m}^{n-r}\left(X, B_{\beta} C(\Delta, L(Y, Z))\right)} \leq(1 / 2)^{n} .
$$

Portanto

$$
a\left(x_{0}+h\right) * b\left(x_{0}+h\right)=\sum_{n=0}^{\infty} q_{n}\left(h^{n}\right)
$$

com obediência a todas às hipóteses do enunciado.

Teorema 3.2.13 Sejam $X, Y, Z$ e $W$ espaços de Banach e $U \subset X$, aberto. $\mathrm{Se}$

$$
x \rightarrow a(x): U \rightarrow B_{\alpha} C(\Delta, L(Z, W))
$$

$e$

$$
x \rightarrow b(x): U \rightarrow B_{\beta} C(\Delta, L(Y, Z))
$$

são analíticas então, se $(a b)(x)=a(x) b(x)$,

$$
x \rightarrow(a b)(x): U \rightarrow B_{\alpha+\beta} C(\Delta, L(Y, W))
$$

é analítica.

$E$ explitamente se $a\left(x_{0}+h\right)=\sum_{n=0}^{\infty} p_{a}^{n}\left(h^{n}\right)$ e $b\left(x_{0}+h\right)=\sum_{n=0}^{\infty} p_{b}^{n}\left(h^{n}\right)$, sendo $p_{a}^{n} \in L_{\text {sym }}^{n}\left(X, B_{\alpha} C(\triangle, L(Z, W))\right.$ e $p_{b}^{n} \in L_{\text {sym }}^{n}\left(X, B_{\beta} C(\triangle, L(Y, Z))\right.$, então

$$
(a b)\left(x_{0}+h\right)=\sum_{n=0}^{\infty} q^{n}\left(h^{n}\right)
$$

com

$$
q^{n}\left(h^{n}\right)=\sum_{0 \leq r \leq n} p_{a}^{r}\left(h^{r}\right) p_{b}^{n-r}\left(h^{n-r}\right)
$$


Prova. Não há grande diferença com a prova precedente, sendo esta muito mais simples.

Por hipótese, $a\left(x_{0}+h\right)=\sum_{n=0}^{\infty} p_{a}^{n}\left(h^{n}\right)$ e $b\left(x_{0}+h\right)=\sum_{n=0}^{\infty} p_{b}^{n}\left(h^{n}\right)$, sendo $p_{a}^{n} \in L_{\text {sym }}^{n}\left(X, B_{\alpha} C(\Delta, L(Z, W))\right.$ e $p_{b}^{n} \in L_{\text {sym }}^{n}\left(X, B_{\beta} C(\Delta, L(Y, Z))\right.$, se $\|h\|_{X}<$ $\delta\left(x_{0}\right)$. Também é válido que, talvez diminuindo um pouco o $\delta$, pelo teorema 3.2.4, $\delta^{n}\left\|p_{a}^{n}\right\|_{L^{n}\left(X, B_{\alpha}(\Delta, L(Z, W))\right)} \leq M_{a}<\infty$ e $\delta^{n}\left\|p_{b}^{n}\right\|_{L^{n}\left(X, B_{\beta}(\Delta, L(Y, W))\right)} \leq M_{b}<$ $\infty$. Admita, sem perda de geralidade, que $M_{a}$ e $M_{b}$ foram escolhidos para que também $M_{a} M_{b} \geq 1$.

Seja

$$
q^{n}\left(h_{1}, \cdots, h_{n}\right):=\sum_{0 \leq r \leq n, \pi} \frac{p_{a}^{r}\left(h_{1}, \cdots, h_{r}\right) p_{b}^{n-r}\left(h_{r+1}, \cdots, h_{n}\right)}{C_{n, r}} .
$$

Portanto, pela proposição $3.2 .9, q^{n} \in L_{\text {sym }}^{n}\left(X, B_{\alpha+\beta} C(\triangle, L(X, Z))\right.$ e se $\|h\|_{X}<$ $\delta /\left(M_{a} M_{b}\right)$, para $n \geq 1$,

$$
\left\|q^{n}\left(h^{n}\right)\right\|_{B_{\alpha+\beta} C(\Delta,, L(X, Z))} \leq(1 / 2)^{n},
$$

As partes que faltam para concluir o argumento podem ser retiradas da prova precedente.

Observação 3.2.14 Ambos teoremas podem ser obtidos bem mais rapidamente a partir de resultados gerais sobre funções analíticas. Especificamente, se $f: U \rightarrow V$ e $g: V \rightarrow Z, X, Y$ e $Z$ espaços de Banach e $U \subset X, V \subset Y$ abertos, são funçôes analíticas então fog $: U \rightarrow Z$ é analítica. Ou seja, composição de funções analíticas fornece uma função analítica. E também se $f: U \rightarrow Y$ e $g: U \rightarrow Z$ são analíticas então o par $(f, g): U \rightarrow Y \times Z$ também é. Finalmente também usando que se uma função é bilinear e contínua então é analítica. A vantagem do nosso esquema é usar essencialmente a definição de função analítica e fornecer as derivadas como subproduto da prova.

\subsection{Analiticidade do Operador de Evolução}

Os passos para obter a analiticidade do operador de evolução $(A, \xi) \rightarrow$ $U_{A}(t, s) \xi$ requerem que façamos a extenção da analiticidade do semigrupo para o caso $(A, \xi) \rightarrow e^{-(t-s) A(s)} \xi$. A analiticida de $A \rightarrow U_{A}$ também é tratada aqui. Mas por simplicidade pensemos só na primeira por enquanto e esqueçamos os espaços. 
Originalmente a idéia consistia em usar o teorema de regularidade do ponto fixo confome descrição em [Henry [8], página 13] e a descrição de operador de evolução de Tanabe. Nesta o operador de evolução é calculado por $U(t, s) \xi=e^{-(t-s) A(s)} \xi+W(t, s) \xi \operatorname{com} W(t, s) \xi=\int_{s}^{t} e^{-(t-\tau) A(\tau)} R(\tau, s) \xi d \tau$. E resta descrever $R$. Chame $k(A)(t, s)=-[A(t)-A(s)] e^{-(t-s) A(s)}$. Então $R$ é a solução de $R(t, s) \xi=k(A)(t, s) \xi+\int_{s}^{t} k(A)(t, \tau) R(\tau, s) \xi d s$.

Se for provada a analiticidade de todas as funções como, por exemplo, de $(A, \xi) \rightarrow e^{-(t-s) A(s)} \xi$, também de $(A, u) \rightarrow \int_{s}^{t} k(A)(t, \tau) u(\tau, s) \xi d s$, então também chega-se à analiticidade de $U(t, s) \xi$, pois o ponto fixo mantém a regularidade da função $F(u):=k(A)(t, s) \xi+\int_{s}^{t} k(A)(t, \tau) u(\tau, s) \xi d s$ se ela for uma contração uniforme em $u$ em uma vizinhança de $A$ [Henry [8], página $13]$.

Entretanto partimos por um caminho que julgamos mais simples que consiste em obter a analiticidade de $U_{A}(t, s) \xi$ com o uso da descrição de operador de evolução de Sobolevskii ou Amann através da série, chamando $a_{A}(t, s) \xi=e^{-(t-s) A(s)} \xi$,

$$
U_{A}(t, s) \xi=a_{A}(t, s) \xi+\left(a_{A} * k_{A}\right)(\xi)(t, s)+\left(a_{A} * k_{A} * k_{A}\right)(\xi)(t, s)+\cdots
$$

em que * denota a convolução, $(f * g)(t, s)=\int_{s}^{t} f(t, \tau) g(\tau, s) d s$.

Possivelmente também este caminho facilite o cálculo das derivadas do operador de evolução.

Proposição 3.3.1 O conjunto $C^{\epsilon}\left([0, T], \mathcal{H}\left(X_{1}, X_{0}\right)\right)$ da definição 3.1 .1 é um subconjunto aberto do espaço de Banach $C^{\epsilon}\left([0, T], L\left(X_{1}, X_{0}\right)\right)$ e cada elemento de $C^{\epsilon}\left([0, T], \mathcal{H}\left(X_{1}, X_{0}\right)\right)$ possui uma vizinhança que também é um conjunto regular. Precisamente, suponha $A \in C^{\epsilon}\left([0, T], \mathcal{H}\left(X_{1}, X_{0}\right)\right)$, com $\left\|(\lambda+A(t))^{-1}\right\| \leq M_{0}(|\lambda|+1)^{-1},\left\|(A(t)-A(s)) A(\tau)^{-1}\right\|_{L\left(X_{0}\right)} \leq a|t-s|^{\epsilon} e$ $D(A(t)) \subset X_{1}$ com constante $a_{1}$ e $X_{1} \subset D(A(t))$ com constante $a_{2}$, sendo que todas as contantes não dependem de $t, s, \tau \in[0, T]$. Então, para $r=$ $\left(a_{1}\left(M_{0}+1\right)^{2}\right)^{-1} / 2$, a bola $B(A, r) \subset C^{\epsilon}\left([0, T], L\left(X_{1}, X_{0}\right)\right)$ está em $\mathcal{A}$ substituindo as constante prévias respectivamente por $2 M_{0}, a_{2}\left[a_{1}\left(M_{0}+1\right)+1\right](r+a)$, $2 a_{1}$ e $a_{2}\left(a_{1}\left(M_{0}+1\right)+1\right)$. E para $P \in B(A, r),\|P(t)-P(s)\|_{L\left(X_{1}, X_{0}\right)} \leq$ $\left(r+a_{2} a\right)|t-s|^{\epsilon}$.

Prova. Todo o argumento da prova de 2.2 .1 pode ser aproveitado.

Obviamente se $A_{0} \in C^{\epsilon}\left([0, T], \mathcal{H}\left(X_{1}, X_{0}\right)\right)$ então $A_{0} \in C^{\epsilon}\left([0, T], L\left(X_{1}, X_{0}\right)\right)$. Chame de $a_{1}$, a constante de imersão de $D\left(A_{0}(t)\right) \subset X_{1}$ e de $a_{2}$ a de $X_{1} \subset$ $D\left(A_{0}(t)\right)$, que não dependem de $t \in[0, T]$. Então se $f \in X_{1}=D\left(A_{0}(t)\right)$, 
$\left\|A_{0}(t) f\right\|_{X_{0}} \leq a_{2}\|f\|_{X_{1}}$ e $A_{0}(t) \in L\left(X_{1}, X_{0}\right),\left\|A_{0}(t)\right\|_{L\left(X_{1}, X_{0}\right)} \leq a_{2}$. E isto também implica que $\left\|A_{0}(t)-A_{0}(s)\right\|_{L\left(X_{1}, X_{0}\right)} \leq a_{0} a_{2}|t-s|^{\epsilon}$.

A bola $B\left(A_{0}, r\right)$ de raio $r$ e centro $A_{0}$ de $C^{\epsilon}\left([0, T], L\left(X_{1}, X_{0}\right)\right)$ é formada pelas operadores $P, t \rightarrow P(t)$, que tem

$$
\begin{aligned}
& \| P \\
& \quad-A_{0} \|_{C^{\epsilon}\left([0, T], L\left(X_{1}, X_{0}\right)\right)} \\
& \quad \sup _{s \in[0, T]}\left\|\left(P-A_{0}\right)(t)\right\|_{L\left(X_{1}, X_{0}\right)} \\
& \quad+\sup _{t, s \in[0, T], t \neq s}\left\|\left[\left(P-A_{0}\right)(t)-\left(P-A_{0}\right)(s)\right] /|t-s|^{\epsilon}\right\|_{L\left(X_{1}, X_{0}\right)}<r .
\end{aligned}
$$

Suponha e $r=\left(a_{1}\left(M_{0}+1\right)^{2}\right)^{-1} / 2 \operatorname{com}\left\|(\lambda+A(t))_{L\left(X_{0}\right)}^{-1}\right\| \leq M_{0} /(|\lambda|+1)$. Então, pela prova da proposição 2.2.1, $\left\|(\lambda+P(t))^{-1}\right\|_{L\left(X_{0}\right)} \leq 2 M_{0} /(|\lambda|+1)$, $D(P(t)) \doteq X_{1}$ uniforme com $t$ e com $P$ na bola $B\left(A_{0}, r\right)$. Imediatamente da definição da norma $C^{\epsilon}\left([0, T], L\left(X_{1}, X_{0}\right)\right)$,

$$
\|(P(t)-P(s))\|_{L\left(X_{1}, X_{0}\right)} \leq\left(r+a_{0}\right)|t-s|^{\epsilon} .
$$

Como, $\left\|P(\tau) A_{0}^{-1}(\tau)\right\|_{L\left(X_{0}\right)} \leq a_{1}\left(M_{0}+1\right)+1$ pela prova da proposição 2.2.1,

$$
\left\|(P(t)-P(s)) P^{-1}(\tau)\right\|_{L\left(X_{1}, X_{0}\right)} \leq a_{2}\left[a_{1}\left(M_{0}+1\right)+1\right]\left(r+a_{0} a_{2}\right)|t-s|^{\epsilon},
$$

concluindo a prova.

Também vai ser útil observar que

$$
\left\|\left[\left(P-A_{0}\right)(t)-\left(P-A_{0}\right)(s)\right] A_{0}^{-1}(s)\right\|_{L\left(X_{0}\right)} \leq r a_{1}\left(M_{0}+1\right)|t-s|^{\epsilon},
$$

pois $\left\|A_{0}^{-1}(\tau)\right\|_{L\left(X_{0}, X_{1}\right)} \leq a_{1}\left(M_{0}+1\right)$ ainda pela prova da proposição 2.2.1.

Proposição 3.3.2 Se $P$ pertence à bola aberta de centro $A_{0}$ e raio $r=$ $\left(a_{1}\left(M_{0}+1\right)^{2}\right)^{-1} / 2$ de $C^{\epsilon}\left([0, T], L\left(X_{1}, X_{0}\right)\right)$ então

$$
\left\|\left[\left(P-A_{0}\right)(t)-\left(P-A_{0}\right)(s)\right] A_{0}^{-1}(s)\right\|_{L\left(X_{0}\right)} \leq r a_{1}\left(M_{0}+1\right)|t-s|^{\epsilon} .
$$

Prova. Última observação da prova da proposição 3.3.1.

Proposição 3.3.3 Suponha $\mathcal{A}$ como em 3.1 .1 e seja $X_{\alpha}:=\left(X_{0}, X_{1}\right)_{\alpha, \infty ; K}$. Então

1.uniformemente com $A \in \mathcal{A}$,

$$
\left\|\left(\lambda+A\left(s_{2}\right)\right)^{-1}-\left(\lambda+A\left(s_{1}\right)\right)^{-1}\right\|_{L\left(X_{0}, X_{\alpha}\right)} \leq c\left|s_{2}-s_{1}\right|^{\epsilon}|\lambda|^{\alpha-1} .
$$


2. uniformemente com $A \in \mathcal{A}, \mathcal{A}$ um conjunto regular, e com $H, H$ pertencente a um limitado de $C^{\epsilon}\left([0, T], L\left(X_{1}, X_{0}\right)\right)$,

$$
\left\|H\left(s_{2}\right)\left(\lambda+A\left(s_{2}\right)\right)^{-1}-H\left(s_{1}\right)\left(\lambda+A\left(s_{1}\right)\right)^{-1}\right\|_{L\left(X_{\alpha}, X_{0}\right)} \leq c\left|s_{2}-s_{1}\right|^{\epsilon}|\lambda|^{-\alpha} .
$$

3. uniformemente com $A \in \mathcal{A}, \mathcal{A}$ conjunto regular, e com $H, H$ pertencente a um limitado de $C^{\epsilon}\left([0, T], L\left(X_{1}, X_{0}\right)\right)$,

$$
\left\|H\left(s_{2}\right)\left(\lambda+A\left(s_{2}\right)\right)^{-1}-H\left(s_{1}\right)\left(\lambda+A\left(s_{1}\right)\right)^{-1}\right\|_{L\left(X_{0}\right)} \leq c\left|s_{2}-s_{1}\right|^{\epsilon} .
$$

Prova. Pela identidada $\left.\lambda+A\left(s_{2}\right)\right)^{-1}-\left(\lambda+A\left(s_{1}\right)\right)^{-1}=\left(\lambda+A\left(s_{1}\right)\right)^{-1}\left(A\left(s_{1}\right)-\right.$ $\left.A\left(s_{2}\right)\right)\left(\lambda+A\left(s_{2}\right)\right)^{-1}$,

$\left\|\left(\lambda+A\left(s_{2}\right)\right)^{-1}-\left(\lambda+A\left(s_{1}\right)\right)^{-1}\right\|_{L\left(X_{0}, X_{\alpha}\right)} \leq\left\|\left(\lambda+A\left(s_{1}\right)\right)^{-1}\right\|_{L\left(X_{0}, X_{\alpha}\right)}\left\|\left(A\left(s_{1}\right)-A\left(s_{2}\right)\right)\right\|_{L\left(X_{1}, X_{0}\right)} \|(\lambda+t$

e aplicação de 1.5 .1 chega-se a 1 .

A identidade

$$
\begin{aligned}
& H\left(s_{2}\right)\left(\lambda+A\left(s_{2}\right)\right)^{-1}-H\left(s_{1}\right)\left(\lambda+A\left(s_{1}\right)\right)^{-1}= \\
& \left(H\left(s_{2}\right)-H\left(s_{1}\right)\right)\left(\lambda+H\left(s_{2}\right)\right)^{-1}+H\left(s_{1}\right)\left(\left(\lambda+A\left(s_{1}\right)\right)^{-1}-\left(\lambda+A\left(s_{2}\right)\right)^{-1}\right)
\end{aligned}
$$

com a identidade e aplicação de 1.5.1 implicam 2.

A prova do remanescente é análoga.

Proposição 3.3.4 Suponha $\mathcal{A}$ como na definição 3.1.1 e seja $X_{\alpha}=\left(X_{0}, X_{1}\right)_{\alpha, \infty ; K}^{o}$ $e \Delta=\{(t, s), 0 \leq s<t \leq T\}$. Suponha também $0 \leq \beta \leq \alpha<1$. Então as funçốes

$$
(A, \xi) \rightarrow\left\{e^{-(t-s) A(s)} \xi, 0 \leq s \leq t \leq T\right\}: C^{\epsilon}\left([0, T], \mathcal{H}\left(X_{1}, X_{0}\right)\right) \times X_{\alpha} \rightarrow C\left(\bar{\Delta}, X_{\alpha}\right)
$$

e

$A \rightarrow\left\{e^{-(t-s) A(s)}, 0 \leq s<t \leq T\right\}: C^{\epsilon}\left([0, T], \mathcal{H}\left(X_{1}, X_{0}\right)\right) \rightarrow B_{\alpha-\beta} C\left(\Delta, L\left(X_{\beta}, X_{\alpha}\right)\right)$

são ambas analíticas.

Prova. Para avaliar a série de cada função procede-se como na parte 1 de 2.2.3. Com o teorema precedente, obtém-se a continuidade requerida para cada termo. 
Proposição 3.3.5 Seja $k(A)(t, s)=-[A(t)-A(s)] e^{-(t-s) A(s)}$. Chame $\Delta=$ $\{(t, s), 0 \leq s<t \leq T\}$. Suponha $\beta \in[0,1)$. Então a função

$A \rightarrow\{k(A)(t, s), 0 \leq s<t \leq T\}: C^{\epsilon}\left([0, T], \mathcal{H}\left(X_{1}, X_{0}\right)\right) \rightarrow B_{1-\beta-\epsilon} C\left(\Delta, L\left(X_{\beta}, X\right)\right)$

é analítica.

Prova. A função $A \rightarrow\{A(t)-A(s), 0 \leq s<t \leq T\}: C^{\epsilon}\left([0, T], \mathcal{H}\left(X_{1}, X_{0}\right)\right) \rightarrow$ $B_{-\epsilon} C\left(\triangle, L\left(X_{1}, X_{0}\right)\right)$ é linear e contínua, portanto, analítica. Então a prova pode ser concluída com a aplicação das proposições 3.3.4 e 3.2.13.

Lema 3.3.6 Suponha $a_{A}(t, s)=e^{-(t-s) A(s)}$ e $k_{A}(t, s)=-[A(t)-A(s)] e^{-(t-s) A(s)}$ - Admita também $0 \leq \beta \leq \alpha<1$. Então em uma vizinhança de $A \in$ $C^{\epsilon}\left([0, T], \mathcal{H}\left(X_{1}, X_{0}\right)\right) \subset C^{\epsilon}\left([0, T], L\left(X_{1}, X_{0}\right)\right)$,

1. $\left\|a_{A}\right\|_{B_{\alpha-\beta} C\left(\Delta, L\left(X_{\beta}, X_{\alpha}\right)\right)} \leq c^{1}$, em que $c^{1}$ é constante na vizinhança e não depende $\alpha, \beta$.

2. $\left\|k_{A}\right\|_{B_{1-\beta-\epsilon} C\left(\Delta, L\left(X_{\beta}, X\right)\right)} \leq c^{2}$, em que $c^{2}$ também é constante na vizinhança e não depende de $\beta$.

Prova. Exceto pela observação da independência das constantes $c^{1}$ e $c^{2}$ com $\alpha$ e $\beta$, o resultado é óbvio, pois as funções $a_{A}$ e $k_{A}$ foram demonstradas analíticas com $A$, portanto contínuas, então, localmente limitadas.

A conclusão da prova consiste em uma aplicação de 2.1.1 e 2.1.4, observando que $k_{A}=[A(t)-A(s)] A^{-1}(s) A(s) e^{-(t-s) A(s)}$ e também que as constantes nestas estimativas podem ser tomadas independentemente de $\alpha, \beta$.

Proposição 3.3.7 Seja $U_{A}(t, s) x$ o operador de evolução para a equação $d u / d t+A(t) u=0, u(s)=x, 0 \leq s \leq t \leq T$. Seja $a_{A}(t, s)=e^{-(t-s) A(s)} e$ $k_{A}(t, s)=-[A(t)-A(s)] e^{-(t-s) A(s)}$.

1. Seu valor pode ser calculado pela série

$$
U_{A}(t, s) x=a_{A}(t, s) x+\left(a_{A} * k_{A}\right)(t, s) x+\left(a_{A} * k_{A} * k_{A}\right)(t, s) x+\cdots,
$$

uniformemente convergente para uma vizinhança de cada $A, A \in C^{\epsilon}\left([0, T], \mathcal{H}\left(X_{1}, X_{0}\right)\right)$.

2. Se $0 \leq \beta \leq \alpha<1$ então série é convergente e uniformemente limitada em $B_{\alpha-\beta} C\left(\Delta, L\left(X_{\beta}, X_{\alpha}\right)\right)$ uniformente em uma vizinhança de cada $A, A \in$ $\mathcal{A} \subset C^{\epsilon}\left([0, T], L\left(X_{1}, X_{0}\right)\right)$. 
3. Suponha $\xi \in X_{\alpha}$. Então a série em $\xi$,

$$
U_{A}(t, s) \xi=a_{A}(t, s) \xi+\left(a_{A} * k_{A}\right)(t, s) \xi+\left(a_{A} * k_{A} * k_{A}\right)(t, s) \xi+\cdots
$$

é convergente e uniformemente limitada em $C\left(\bar{\Delta}, X_{\alpha}\right)$ uniformemente em uma vizinhança de cada $A, A \in \mathcal{A} \subset C^{\epsilon}\left([0, T], L\left(X_{1}, X_{0}\right)\right)$.

Prova. A primeira parte foi provada em [Sobolevskii[1]] e em [Amann[5]] exceto pela observação que incluímos de que há convergência uniforme em uma vizinhança de $A$ na norma de $X_{0}$. Para ver que isto ocorre é suficiente notar que todas as estimativas em Sobolevskii ou Amann que levam à prova da convergência dependem somente das constantes que ocorrem em $\mathcal{A}$ da definição 3.1.1. Em [Tanabe [2],páginas 118 e 119] é possível também ver a mesma independêndencia num argumento curto.

Para provar a segunda parte, reescreva a série como

$$
a_{A}+a_{A} * k_{A}+a_{A} *\left(k_{A}+k_{A} * k_{A}+\cdots\right) * k_{A} .
$$

Chame $\omega_{A}:=k_{A}+k_{A} * k_{A}+\cdots$ Em [Amann[5], página 50, lema 3.2.1] está provado que

$$
\left\|\omega_{A}(t, s)\right\|_{L(X)} \leq(t-s)^{\epsilon-1} c_{k} c(\epsilon, \mu) \Gamma(\epsilon) e^{(1+\mu)\left(\Gamma(\epsilon) c_{k}\right)^{1 / \epsilon}(t-s)},
$$

com $c_{k}=\left\|k_{A}\right\|_{B_{1-\epsilon} C(\Delta, L(X))}$ e um arbitrário $\mu>0$, sendo esta estimativa uniforme em uma vizinhança de $A \in \mathcal{A} \subset C^{\epsilon}\left([0, T], L\left(X_{1}, X_{0}\right)\right)$, pois

$$
\left\|k_{A}\right\|_{L(X)} \leq a c_{1}(t-s)^{\epsilon-1}
$$

de acordo também com 1.2.19 e com a definição de $\mathcal{A}$ em 3.1.1.

Para concluir, primeiramente note que $a_{A} \in B_{\alpha-\beta} C\left(\Delta, L\left(X_{\beta}, X_{\alpha}\right)\right)$ e, aplicando 3.2.11, $a_{A} * k_{A} \in B_{\alpha-\beta-\epsilon} C\left(\Delta, L\left(X_{\beta}, X_{\alpha}\right)\right) \mathrm{e}$

$$
a_{A} * \omega_{A} * k_{A} \in B_{\alpha-\beta-2 \epsilon} C\left(\Delta, L\left(X_{\beta}, X_{\alpha}\right)\right) .
$$

Como valem as imersões

$$
B_{\alpha-\beta-\epsilon} C\left(\Delta, L\left(X_{\beta}, X_{\alpha}\right) \subset B_{\alpha-\beta} C\left(\Delta, L\left(X_{\beta}, X_{\alpha}\right)\right)\right.
$$

e

$$
B_{\alpha-\beta-2 \epsilon} C\left(\triangle, L\left(X_{\beta}, X_{\alpha}\right)\right) \subset B_{\alpha-\beta} C\left(\Delta, L\left(X_{\beta}, X_{\alpha}\right)\right)
$$




\section{CAPÍTULO 3. ESTIMATIVASE ANALITICIDADE DO OPERADOR DE EVOLUÇÄ}

continuamente, com constantes de imersão $T^{\epsilon}$ e $T^{2 \epsilon}$, então a série é convergente em $B_{\alpha-\beta} C\left(\triangle, L\left(X_{\beta}, X_{\alpha}\right)\right)$.

Precisamente, pela proposição 3.2.11, a série pode ser estimada por

$$
\begin{aligned}
& \left\|U_{A}\right\|_{B_{\alpha-\beta} C\left(\Delta, L\left(X_{\beta}, X_{\alpha}\right)\right)} \leq\left\|a_{A}\right\|_{B_{\alpha-\beta} C\left(\Delta, L\left(X_{\beta}, X_{\alpha}\right)\right)} \\
& \quad+T^{\epsilon} B(\alpha, 1-\beta-\epsilon)\left\|a_{A}\right\|_{B_{\alpha} C\left(\Delta, L\left(X, X_{\alpha}\right)\right)}\left\|k_{A}\right\|_{B_{1-\beta-\epsilon} C\left(\Delta, L\left(X_{\beta}, X\right)\right)} \\
& \quad+c T^{2 \epsilon} B(1-\epsilon, 1+\beta-\epsilon) B(\alpha, 1+\beta-2 \epsilon),
\end{aligned}
$$

onde $c=\left\|a_{A}\right\|_{B_{\alpha} C\left(\Delta, L\left(X, X_{\alpha}\right)\right)}\left\|\omega_{A}\right\|_{B_{1-\epsilon} C(\Delta, L(X))}\left\|k_{A}\right\|_{B_{1-\beta-\epsilon} C\left(\Delta, L\left(X_{\beta}, X\right)\right)}$.

Todas as normas do último majorante são limitados em uma vizinhança de cada $A$ conforme lema 3.3.6.

Quanto à terceita parte, para começar, $(t, s) \rightarrow a_{A}(t, s) \xi$ pertence à $C\left(\bar{\Delta}, X_{\alpha}\right)$ conforme proposição 3.3.4. Há melhora da regularidade nos demais termos. Temos $a_{A} * k_{A} \in B_{-\epsilon} C\left(\Delta, L\left(X_{\alpha}\right)\right)$, mas este espaço está imerso continuamente com constante $T^{\epsilon}$ em $C\left(\bar{\Delta}, L\left(X_{\alpha}\right)\right)$ tomando valor zero para $t=s$. No nosso caso, $a_{A} * k_{A}(t, t)=0$ por definição e então $a_{A} * k_{A} \in C\left(\bar{\Delta}, L\left(X_{\alpha}\right)\right)$. Mas isto é muito mais do que desejamos, uma vez que só queremos provar que $\left(a_{A} * k_{A}\right) \xi,\left(\left(a_{A} * k_{A}\right) \xi\right)(t, s)=\left(a_{A} * k_{A}\right)(t, s) \xi$, está em $C\left(\bar{\Delta}, X_{\alpha}\right)$. Mesmo argumento para $\left(a_{A} * \omega_{A} * k_{A}\right) \xi$.

Já foi provada a convergência e limitação da série $U_{A}$ em $B_{0} C\left(\Delta, L\left(X_{\alpha}\right)\right)$ o que é mais que o necessário para termos as mesmas propriedades para a série da terceira parte em $C\left(\bar{\Delta}, X_{\alpha}\right)$, pois, no caso que falta, esta série vale $\xi$.

Observação 3.3.8 O pior de todos é $a_{A}$.

Teorema 3.3.9 Suponha $0 \leq \beta \leq \alpha<1$. As funções

$$
\begin{aligned}
& A \rightarrow\left\{U_{A}(t, s), 0 \leq s<t \leq T\right\}: C^{\epsilon}\left([0, T], \mathcal{H}\left(X_{1}, X_{0}\right)\right) \rightarrow B_{\alpha-\beta} C\left(\Delta, L\left(X_{\beta}, X_{\alpha}\right)\right), \\
& (A, \xi) \rightarrow\left\{U_{A}(t, s) \xi, 0 \leq s \leq t \leq T\right\}: C^{\epsilon}\left([0, T], \mathcal{H}\left(X_{1}, X_{0}\right)\right) \times X_{\alpha} \rightarrow C\left(\bar{\Delta}, X_{\alpha}\right), \\
& \text { e } \\
& (A, f) \rightarrow\left\{\int_{s}^{t} U_{A}(t, \tau) f(\tau) d \tau, 0 \leq s \leq t \leq T\right\}: \\
& C^{\epsilon}\left([0, T], \mathcal{H}\left(X_{1}, X_{0}\right)\right) \times C\left([0, T], X_{\beta}\right) \rightarrow C\left(\bar{\Delta}, X_{\alpha}\right)
\end{aligned}
$$

são todas analíticas. 
Prova. Os dois primeiros são consequência da convergência uniforme da série do operador de evolução, teorema 3.3.7, e da analiticidade de cada termo, pela 3.3 .4 e pela 3.2 .12 .

Remodelemos a última afirmação para recairmos nos teoremas disponíveis. Se $f \in C\left([0, T], X_{\beta}\right)$ então definindo $F(\lambda)(t, s)=f(t) \lambda, F \in B_{0} C\left(\Delta, L\left(C, X_{\beta}\right)\right)$. Então pela primeira parte e pelo teorema $3.2 .11, \mathcal{A} \ni A \rightarrow U_{A} * F \in$ $B_{\alpha-\beta-1} C\left(\triangle, L\left(C, X_{\alpha}\right)\right)$ é analítica. Como $\alpha-\beta-1<0, A \rightarrow U_{A} * F \in$ $C\left(\bar{\triangle}, L\left(C, X_{\alpha}\right)\right)$ também é analítica. A análise da série de $U_{A} * F$ no caso particular $\left(U_{A} * F(1)\right)$ fornece a analiticidade requerida em relação à $A$.

Mas em relação à $f$ a função é linear e contínua, pois $\int_{s}^{t} U_{A}(t, \tau) f(\tau) d \tau$ pertence à $C\left(\bar{\Delta}, X_{\alpha}\right)$ pelo que acabamos de ver e

$\left\|\int_{s}^{t} U_{A}(t, \tau) f(\tau) d \tau\right\|_{C\left(\bar{\Delta}, X_{\alpha}\right)} \leq(t-s)^{\beta-\alpha+1}\left\|U_{A}\right\|_{B_{\alpha-\beta} C\left(\Delta, L\left(X_{\beta}, X_{\alpha}\right)\right)}\|f\|_{C\left([0, T], X_{\beta}\right)}$.

\subsection{Estimativas para o operador de evolução}

As estimativas para o operador de evolução $U_{A}$ ocorrem nos espaços $\left(X_{0}, X_{1}\right)_{\alpha, \infty ; K}$. Mas para efetuar os cálculos usamos a igualdade destes espaços com os espaços $X_{\alpha, 1 ; \infty}^{\prime}(A)$. Para melhor definição das constantes das estimativas que vão surgir quando fazemos esta troca, uma preparação prévia é necessária.

Proposição 3.4.1 Seja $\mathcal{A} \subset C^{\epsilon}\left([0, T], \mathcal{H}\left(X_{1}, X_{0}\right)\right)$ um conjunto regular. $E$ sejam $X_{\alpha}(A)=X_{\alpha, 1 ; \infty}(A), A \in \mathcal{A}$ e $X_{\alpha, K}=\left(X_{0}, X_{1}\right)_{\alpha, \infty ; K}$. Então se um operador linear $T$ obedece $\|T\|_{L\left(X_{\beta}, X_{\alpha}\right)} \leq c$ também obedece

$$
\|T\|_{L\left(X_{\beta, K}, X_{\alpha, K}\right)} \leq \operatorname{cmax}\left\{1+1 / \alpha, c_{0} \delta^{\alpha-1} / e\right\}\left(1+\max \left\{c_{0}, c_{1}\right\}\right)
$$

quando $\alpha \in(0,1]$ e $\beta \in[0,1]$. E para $\alpha=0$,

$$
\|T\|_{L\left(X_{\beta, K}, X_{0, K}\right)}=\|T\|_{L\left(X_{\beta, K}, X\right)} \leq c\left(1+\max \left\{c_{0}, c_{1}\right\}\right) .
$$

Prova. A conclusão resulta da equivalência das normas dos espaços.

Os teoremas 1.2 .20 e 1.2 .27 com suas demonstrações fornecem

$$
\left(X_{0}, X_{1}\right)_{\alpha, \infty ; K} \subset X_{\alpha, 1 ; \infty}^{\prime}(A)
$$

com constante de imersão $1+\max \left\{c_{0}, c_{1}\right\}$. Por outro lado

$$
X_{\alpha, 1 ; \infty}^{\prime}(A) \subset\left(X_{0}, X_{1}\right)_{\alpha, \infty ; K}
$$

com constante de imersão $\max \left\{1+1 / \alpha, c_{0} \delta^{\alpha-1} / e\right\}$ se $\alpha \in(0,1]$ e 1 se $\alpha=0$. 
Notação 3.4.2 Para um conjunto regular $\mathcal{A} \subset C^{\epsilon}\left([0, T], \mathcal{H}\left(X_{1}, X_{0}\right)\right)$, chamamos

$$
i(\alpha)=\max \left\{1+1 / \alpha, c_{0} \delta^{\alpha-1} / e\right\}\left(1+\max \left\{c_{0}, c_{1}\right\}\right)
$$

se $\alpha \in(0,1]$ e $i(0)=\left(1+\max \left\{c_{0}, c_{1}\right\}\right)$.

Teorema 3.4.3 Suponha $X_{\alpha}:=\left(X_{0}, X_{1}\right)_{\alpha, \infty ; K}$. Esuponha $A \in \mathcal{A}, \mathcal{A} \subset$ $C^{\epsilon}\left([0, T], \mathcal{H}\left(X_{1}, X_{0}\right)\right)$ um conjunto regular como em 3.1.1. Então

1. quando $\beta \in[0,1]$,

$$
\left\|A(t) U_{A}(t, s)\right\|_{L\left(X_{\beta}, X\right)} \leq c(\beta)|t-s|^{\beta-1},
$$

$c(\beta)=c_{G}\left(2 c_{0}, 2 a c_{0}, 1-\beta, 1-\epsilon\right) i(0)$.

2.quando $\beta \in[0,1]$,

$$
\left\|U_{A}(t, s)\right\|_{L\left(X_{\beta}, X_{1}\right)} \leq c(\beta)|t-s|^{\beta-1}
$$

com $c(\beta)=c^{1}\left(T^{1-\beta}+c^{\prime}(\beta)\right), c^{1}$ a constante de imersấo $X_{1} \subset D(A(t))$ e $c^{\prime}(\beta)$ definido no item 1.

3.

$$
\left\|U_{A}(t, s)\right\|_{L\left(X_{\beta}, X_{\alpha}\right)} \leq c(\alpha, \beta)|t-s|^{\beta-\alpha},
$$

quando $0 \leq \beta \leq \alpha<1$, com $c(\alpha, \beta)=i(\alpha) \max \left\{c_{1} T^{\alpha-\beta}, a c_{0} c_{G} B(1+\epsilon-\right.$ $\left.\alpha, \beta) T^{\epsilon}, c_{0}\right\}$ para $\beta \in(0,1]$ e $c(\alpha, 0)=i(\alpha) \max \left\{c(\alpha), c^{1} c(\alpha) B(1-\alpha, \epsilon) T^{\epsilon}\right\}$ e $c(\alpha)=\max \left\{\left(2 \alpha e^{-1}\right)^{\alpha}, 2\right\}, c^{1}$ definido em [Sobolevskii, (1.28)]. O caso $\alpha=1$ também vale com a constante do item 2.

Prova. Com a fórmula de 3.1.4 e a estimativa para o semigrupo em 2.1.4, $\left\|A(t) U_{A}(t, s) x\right\| \leq 2 c_{0}(t-s)^{\beta-1} e^{-\frac{\delta}{2}(t-s)}\|x\|_{\beta, 1 ; \infty}+2 a c_{0} \int_{s}^{t}(t-\tau)^{\epsilon-1}\left\|A(\tau) U_{A}(\tau, s) x\right\| d \tau$.

Aplicação da desigualdade de Gronwall implica a primeira estimativa. A segunda estimativa resulta do fato de que $X_{1}$ está continuamente imerso em $D(A(t)$.

A prova da última parte parte requer a primeira parte. Sobolevskii[[1]] também procede assim, mas o argumento com espaços intermediários é menos direto que o argumento com potências fracionárias. 
Seja $x \in X_{\beta}, \beta \in(0,1]$. Pela igualdade em 3.1.4, calculada em $h^{1-\alpha} A(t) e^{-h A(t)}$, $0<h<\infty$,

$$
\begin{aligned}
& h^{1-\alpha} A(t) e^{-h A(t)} U_{A}(t, \tau) x \\
& \quad=h^{1-\alpha} A(t) e^{-h A(t)} e^{-(t-\tau) A(t)} x+ \\
& \quad \int_{\tau}^{t} h^{1-\alpha} A(t) e^{-h A(t)} e^{-(t-\tau) A(t)}(A(t)-A(s)) A^{-1}(s) A(s) U_{A}(s, \tau) x d s .
\end{aligned}
$$

Cada parcela pode ser estimatima em função da norma de $x$ em $X_{\beta, 1 ; \infty}$.

Para a primeira parcela, é necessário separar o cálculo em dois casos. Quando $t-\tau \leq h$, rearranjamo-la como $e^{-(t-\tau) A(t)} h^{\beta-\alpha} h^{1-\beta} A(t) e^{-h A(t)} x$ e quando $t-\tau \geq h$, como $h^{1-\alpha} e^{-h A(t)}(t-\tau)^{\beta-1}(t-\tau)^{1-\beta} A(t) e^{-(t-\tau) A(t)} x$. Portanto,

$$
\sup _{0<s<\infty}\left(\left\|h^{1-\alpha} A(t) e^{-h A(t)} e^{-(t-\tau) A(t)} x\right\|\right) \leq c_{0}(t-\tau)^{\beta-\alpha} \sup _{0<h<\infty}\left(h^{1-\beta}\left\|A(t) e^{-h A(t)} x\right\|\right) .
$$

A norma em $X$ da segunda parcela com a ajuda do primeiro item não excede

$a c_{0} c_{G} \int_{\tau}^{t}(t-s)^{\epsilon-\alpha}(s-\tau)^{\beta-1} d s\|x\|_{X_{\beta, 1 ; \infty}(A(t))}=a c_{0} c_{G} B(1+\epsilon-\alpha, \beta)(t-\tau)^{\beta-\alpha+\epsilon}\|x\|_{X_{\beta, 1 ; \infty}(A(t))}$.

Para a norma em $X$ de $U(t, \tau) x,\left\|U_{A}(t, \tau) x\right\| \leq c_{1}\|x\|$.

A reunião de todas estas estimativas implica

$\|U(t, \tau) x\|_{X_{\alpha, 1 ; \infty}(A(t))} \leq \max \left\{c_{1} T^{\alpha-\beta}, a c_{0} c_{G} B(1+\epsilon-\alpha, \beta) T^{\epsilon}, c_{0}\right\}\|x\|_{X_{\beta, 1 ; \infty}(A(t))}$.

Para o caso $\beta=0$, recorremos à [Sobolevskii[1], (1.14)] que fornece a relação

$$
U_{A}(t, \tau)=e^{-(t-\tau) A(\tau)}+\int_{\tau}^{t} e^{-(t-s) A(s)} \Phi(s, \tau) d s,
$$

com $\|\Phi(s, \tau)\|_{L(X)} \leq c^{1}|t-\tau|^{\epsilon-1}$, segundo [Sobolevskii[1], (1.28)]. Portanto,

$$
\|U(t, \tau) x\| \leq c(\alpha)(t-\tau)^{-\alpha} x+\int_{\tau}^{t} c(\alpha) c^{1}(t-s)^{-\alpha}(s-\tau)^{\epsilon-1} x d s,
$$

em que, conforme 2.1.1, $c(\alpha)=c_{0} \max \left\{\left(2 \alpha e^{-1} / \delta\right)^{\alpha}, 2\right\}$. Após integração, a prova está concluída, exceto pelo caso $\alpha=1$. Mas este caso é consequência direta da imersão $X_{1} \subset\left(X_{0}, X_{1}\right)_{1, \infty ; K}$ e do item anterior. 


\section{CAPÍTULO 3. ESTIMATIVAS E ANALITICIDADE DO OPERADOR DE EVOLUÇÂ}

Teorema 3.4.4 Suponha $X_{\alpha}:=\left(X_{0}, X_{1}\right)_{\alpha, \infty ; K}$ e $A \in \mathcal{A}, \mathcal{A}$ como em 3.1.1 um conjunto regular. Então

$$
\left\|U_{A}(t, s)-I\right\|_{L\left(X_{\beta}, X_{\alpha}\right)} \leq c(\alpha, \beta)(t-s)^{\beta-\alpha},
$$

quando $0 \leq \alpha \leq \beta \leq 1,0 \leq s \leq t \leq T$.

Prova. Omita $A$ em $U_{A}$ por simplicidade. $O$ caso $\beta=0$ e, portanto, $\alpha=0$, é simplesmente a uniformidade da norma $L(X)$ de $U(t, \tau)$ em $0 \leq \tau \leq t \leq T$ [Sobolevskii , página 5 , teorema $2,(0.16)$ ]. h).

Suponha então $\beta>0$ e $x \in\left(X_{0}, X_{1}\right)_{\beta, \infty ; K} \doteq X_{\beta, 1 ; \infty}(A(t)) \doteq X_{\beta, 1 ; \infty}(A(t+$

Pela fórmula em 3.1.4

$$
(U(t+h, t)-I) x=\left(e^{-h A(t+h)}-I\right) x+\int_{t}^{t+h} e^{-(t+h-s) A(t+h)}(A(t+h)-A(s)) U(s, t) x d s
$$

Para avaliar a norma de $U(t+h, t)-I$ em $X_{\alpha, 1 ; \infty}(A(t)$ só precisamos nos preocupar com a parte em sup $0<r<\infty$, pois a norma $\|U(t+h, t)-I\|_{L(X)}$ é unifomemente limitada. Adicionalmente, a parcela $e^{-h A(t+h)}-I$ já foi avaliada em ?? e, portanto, é necessário apenas avaliar

$$
\sup _{0<r<\infty}\left(r^{1-\alpha} \| A(t+h) e^{-r A(t+h)} \int_{t}^{t+h} e^{-(t+h-s) A(t+h)}(A(t+h)-A(s)) U(s, t) x d s\right) \| .
$$

A eliminação da dependência com $r$ pode ser feita com a separação

$$
\begin{aligned}
& \left\|A(t+h) e^{-r A(t+h)} e^{-(t+h-s) A(t+h)}\right\| \\
& \quad \leq\left\|A(t+h) e^{-r A(t+h)}\right\|_{L\left(X_{\alpha}, X\right)}\left\|e^{-(t+h-s) A(t+h)}\right\|_{L\left(X, X_{\alpha}\right)} .
\end{aligned}
$$

Com a aplicação de 2.1.1 e 2.1.4, $\left\|A(t+h) e^{-r A(t+h)} e^{-(t+h-s) A(t+h)}\right\| \leq c(\alpha) r^{\alpha-1}(t+$ $h-s)^{-\alpha}$.

Também com 3.4.3,

$$
\begin{aligned}
& r^{1-\alpha}\left\|A(t+h) e^{-r A(t+h)} \int_{t}^{t+h} e^{-(t+h-s) A(t+h)}(A(t+h)-A(s)) U(s, t) x d s\right\| \\
& \leq c(\alpha, \beta) \int_{t}^{t+h}(t+h-s)^{\epsilon-\alpha}(s-t)^{\beta-1} d s\|x\|_{X_{\beta}}
\end{aligned}
$$

e $\int_{t}^{t+h}(t+h-s)^{\epsilon-\alpha}(s-t)^{\beta-1} d s=B(1+\epsilon-\alpha, \beta) h^{\beta-\alpha+\epsilon}$, para concluir a prova. 
Observação 3.4.5 $\mathrm{Na}$ última integral, há uma sobra de regularidade, isto é, a integral é finita até quando $\alpha<1+\epsilon$. Não podemos chegar a este $X_{\alpha}$ porque a igualdade entre este espaço descrito com o método $K$ e os demais métodos descritos no capítulo 1 é garantida somente para $\alpha \in[0,1]$.

Teorema 3.4.6 Suponha $A \in \mathcal{A}, \mathcal{A}$ como em 3.1 .1 um conjunto regular. $E$ chame $X_{\alpha}:=\left(X_{0}, X_{1}\right)_{\alpha, \infty ; K}$. Então se $\xi \in\left(X_{0}, X_{1}\right)_{\alpha, \infty ; K}^{o}$

$$
(t-s)^{1-\alpha}\left\|A(t) U_{A}(t, s) \xi\right\| \rightarrow 0
$$

$e$

$$
\left\|\left(U_{A}(t, s)-I\right) \xi\right\|_{X_{\alpha}} \rightarrow 0
$$

se $t \rightarrow s, t \geq s$.

Prova. A aplicação da desigualdade de Gronwall segundo a demonstração em 3.4.3 implica

$$
\left\|A(t) U_{A}(t, s) \xi\right\| \leq c_{G}\left(1, a c_{1}, 0,1-\epsilon\right)\left\|A(t) e^{-(t-s) A(t)} \xi\right\| .
$$

Mas pela hipótese, $(t-\tau)^{1-\alpha}\left\|A(t) e^{-(t-s) A(t)} \xi\right\|$ é $o(1)$ quando $t-s \rightarrow 0+$, justificando a primeira estimativa.

Novamente pela fórmula em 3.1.4

$$
\left(U_{A}(t, s)-I\right) \xi=\left(e^{-(t-s) A(t)}-I\right) \xi+\int_{s}^{t} e^{-(t-\tau) A(t)}(A(t)-A(s)) U_{A}(\tau, s) \xi d s .
$$

Conforme resulta da demonstração em 3.4 .4

$$
\left\|\int_{s}^{t} e^{-(t-\tau) A(t)}(A(t)-A(\tau)) U_{A}(\tau, s) \xi d s\right\|_{X_{\alpha}} \leq c(\alpha)(t-s)^{\epsilon}\|\xi\|_{X_{\alpha}}
$$

que obviamente se comporta como o(1) quando $t-s \rightarrow 0+$. Note que $\left\|\int_{s}^{t} e^{-(t-\tau) A(t)}(A(t)-A(s)) U_{A}(\tau, s) \xi d s\right\|_{X_{0}} \leq c(\alpha) \int_{s}^{t}(t-\tau)^{\epsilon}(\tau-s)^{\alpha-1}\|\xi\|_{X_{\alpha}} d s$.

Então para concluir o argumento, é suficiente notar que $\left\|\left(e^{-(t-s) A(t)}-I\right) \xi\right\|_{X_{\alpha}^{\circ}}$ é $o(1)$ quando $t-s \rightarrow 0+$, consequência de que $\xi \in\left(X_{0}, X_{1}\right)_{\alpha, \infty}^{o}$ por hipótese. Veja 1.2.40. 


\section{CAPÍTULO 3. ESTIMATIVAS E ANALITICIDADE DO OPERADOR DE EVOLUÇÃ}

Teorema 3.4.7 Suponha $A \in \mathcal{A}, \mathcal{A}$ como em 3.1.1, um conjunto regular e $X_{\alpha}:=\left(X_{0}, X_{1}\right)_{\alpha, \infty ; K}$. Entã o

$\left\|U_{A}(t, \tau)-U_{A}(s, \tau)\right\|_{L\left(X_{\beta}, X_{\alpha}\right)} \leq c(\alpha, \beta, \gamma)|t-s|^{\gamma-\alpha} \max \left\{(s-\tau)^{\beta-\gamma},(t-\tau)^{\beta-\gamma}\right\}$

quando $0 \leq \alpha \leq \gamma \leq 1,0 \leq \beta \leq \gamma \leq 1,0 \leq \tau \leq t, s \leq T$.

Prova. Suponha $t \geq s$. O outro caso é análogo.

A propriedade básica do operador de evolução $U_{A}(t, \tau)=U_{A}(t, s) U_{A}(s, \tau)$ permite concluir a estimativa diretamente dos teoremas 3.4.3 e 3.4.4. Realmente $U_{A}(t, \tau)-U_{A}(s, \tau)=\left(U_{A}(t, s)-I\right) U_{A}(s, \tau)$ e, então, $\left\|U_{A}(t, \tau)-U_{A}(s, \tau)\right\|_{L\left(X_{\beta}, X_{\alpha}\right)} \leq$ $\left\|U_{A}(t, s)-I\right\|_{L\left(X_{\gamma}, X_{\alpha}\right)}\left\|U_{A}(s, \tau)\right\|_{L\left(X_{\beta}, X_{\gamma}\right)}$.

Teorema 3.4.8 Suponha $A \in \mathcal{A}, \mathcal{A}$ como em 3.1.1, um conjunto regular $e$ $X_{\alpha}:=\left(X_{0}, X_{1}\right)_{\alpha, \infty ; K}$. Então

$\left\|U_{A}(t, s)-U_{A}(t, \tau)\right\|_{L\left(X_{\beta}, X_{\alpha}\right)} \leq c(\alpha, \beta, \gamma)|s-\tau|^{\beta-\gamma} \max \left\{(t-s)^{\gamma-\alpha},(t-\tau)^{\gamma-\alpha}\right\}$

quando $0 \leq \gamma \leq \beta \leq 1$ e $0 \leq \gamma \leq \alpha \leq 1,0 \leq \tau, s \leq t \leq T$.

Prova. Suponha $s \geq \tau$. O outro caso é análogo.

Pela propriedade fundamental do operador de evolução $U_{A}(t, \tau)=U_{A}(t, s) U_{A}(s, \tau)$ e, então, $U_{A}(t, s)-U_{A}(t, \tau)=U_{A}(t, s)\left(I-U_{A}(s, \tau)\right)$.

Adaptação do argumento final da demonstração do teorema 3.4.8 conclui a prova.

Teorema 3.4.9 Suponha $\mathcal{A} \subset C^{\epsilon}\left([0, T], L\left(X_{1}, X_{0}\right)\right)$ um conjunto regular conforme 3.1.1. Chame $X_{\beta}=\left(X_{0}, X_{1}\right)_{\beta, \infty, K}$. Então uniformemente em $\mathcal{A}$,

$$
\left\|A^{\gamma}(t) U_{A}(t, \tau)\right\|_{L\left(X_{\beta}, X_{0}\right)} \leq c(\beta, \gamma)(t-\tau)^{\beta-\gamma}
$$

quando $0 \leq \beta \leq 1, \beta \leq \gamma<1+\epsilon$.

Prova. O caso $\beta=0$ já foi demonstrado por [Sobolevskii[1], página 23, (1.65)].

Admita então $\beta>0$. O teorema 3.1.4 nos fornece

$$
\begin{aligned}
& \left\|A^{\gamma}(t) U_{A}(t, \tau)\right\|_{L\left(X_{\beta}, X_{0}\right)} \\
& \quad \leq\left\|A^{\gamma}(t) e^{-(t-\tau) A(\tau)}\right\|_{L\left(X_{\beta}, X_{0}\right)}+ \\
& \quad \int_{\tau}^{t}\left\|A^{\gamma}(t) e^{-(t-s) A(s)}\right\|_{L\left(X_{0}\right)}\left\|(A(t)-A(s)) A^{-1}(s)\right\|_{L\left(X_{0}\right)}\left\|A(s) U_{A}(s, \tau)\right\|_{L\left(X_{\beta}, X_{0}\right)} .
\end{aligned}
$$


Então,

$$
\left\|A^{\gamma}(t) U(t, \tau)\right\|_{L\left(X_{\beta}, X_{0}\right)} \leq c(\beta, \gamma)(t-\tau)^{\beta-\gamma}+\int_{\tau}^{t}(t-s)^{-\gamma+\epsilon}(s-\tau)^{\beta-1} d s .
$$

Sob as restrições dadas, a última integral vale $B(1+\epsilon-\gamma, \beta)(t-\tau)^{\beta+\epsilon-\gamma}$.

Teorema 3.4.10 Suponha $\mathcal{A} \in C^{\epsilon}\left([0, T], \mathcal{H}\left(X_{1}, X_{0}\right)\right)$ um conjunto regular. Chame $X_{\beta}=\left(X_{0}, X_{1}\right)_{\beta, \infty, K}$. Então uniformemente em $\mathcal{A}$,

1. quando $1 \leq \gamma<1+\epsilon, 0 \leq \alpha \leq 1,0 \leq \beta \leq 1$

$$
\left\|A^{\alpha}(t+h, \tau)[U(t+h)-U(t, \tau))\right\|_{L\left(X_{\beta}, X_{0}\right)} \leq c(\alpha, \beta, \gamma) h^{\gamma-\alpha}(t-\tau)^{\beta-\gamma}
$$

2. quando $1 \leq \gamma<1+\epsilon, 0 \leq \alpha \leq 1,0 \leq \beta \leq 1$

$$
\| U(t+h, \tau)-U(t, \tau)) \|_{L\left(X_{\beta}, X_{\alpha}\right)} \leq c(\alpha, \beta, \gamma) h^{\gamma-\alpha}(t-\tau)^{\beta-\gamma}
$$

Prova. O cálculo utiliza pesadamente as estimativas de Sobolevskii. Requer uma adaptação cuidadosa do argumento que justifica a estimativa correspondente com potências de operadores, a [[1], página 25, (1.28)].

Chame $U_{A}=U$.

A propriedade básica do operador de evolução implica a identidade

$$
\begin{aligned}
U(t & +h, \tau)-U(t, \tau) \\
& =\left\{\left[U(t+h, t)-e^{-h A(t+h)}\right] A^{-\gamma}(t)-\int_{0}^{h} A^{1-\gamma}(t+h) e^{-s A(t+h)} d s\right. \\
& \left.+\int_{0}^{h} A(t+h) e^{-s A(t+h)} d s\left[A^{-\gamma}(t+h)-A^{-\gamma}(t)\right]\right\} A^{\gamma}(t) U(t, \tau) .
\end{aligned}
$$

As estimativas de Sobolevskii no caso particular que nos interessa são as seguintes.

Para $\gamma \geq 1$, em [[1], página $23,(1.64)]$,

$$
\left\|A(t)\left[A^{-\gamma}(t)-A^{-\gamma}(\tau)\right]\right\| \leq c(\gamma)|t-\tau|^{\epsilon} .
$$

Para $\alpha \in[0,1]$ e $\beta \in[0,1], \xi \in[0, T]$, em [[1], página 24, (1.67)],

$$
\left\|A^{\alpha}(\xi)\left[U(t, \tau)-e^{-(t-\tau) A(t)}\right] A^{-\gamma}(\tau)\right\| \leq c(\alpha, \beta)|t-\tau|^{\epsilon+\beta-\alpha} .
$$

Então $A(t+h)(U(t+h, \tau)-U(t, \tau))$ na norma $L\left(X_{\beta}, X_{0}\right)$ não excede $c(\alpha, \beta)\left[h^{\gamma-\alpha+\epsilon}+\int_{0}^{h} s^{\gamma-1-\alpha} d s+\int_{0}^{h} s^{-\alpha} d s h^{\epsilon}\right](t-\tau)^{\beta-\gamma}$.

$\mathrm{O}$ outro item segue da imersão $X^{\alpha}(A(t+h)) \subset X_{\alpha}$. 
Observação 3.4.11 Em vez de $A(t+h)^{\alpha}$, Sobolevskii usa $A(\xi)^{\alpha}$. No meu entender o preço desta liberdade implica substituir o $\alpha$ do lado direito por um $\alpha^{\prime}>\alpha$. O motivo: há garantia de que $\| A^{\alpha}(t) A^{-\alpha^{\prime}}(\tau \| \leq c(\alpha, \beta)$ se $0 \leq \alpha<\alpha^{\prime} \leq 1$, por [[1], 22, (1.59)], exclusive $\alpha^{\prime}=\alpha$. Nominalmente, diz-se que o operador $A^{\alpha}(t)$ é subordinado ao operador $A^{\alpha^{\prime}}(t)$. 


\section{Capítulo 4}

\section{Elementos da teoria linear}

Em muitos lugares, é possível achar resultados sobre a teoria linear da equação parabólica. Especificamente para a equação $d u / d t+A(t) u=f(t)$, $t>\tau$ onde $A(t)$ gera um semigrupo analítico em um espaço de Banach $X_{0}$ e $D(A(t)) \doteq X_{1}$ com $X_{1}$ um espaço de Banach continua e densamente imerso em $X_{0}$.

Nosso bibliografia básica aqui é o trabalho de [Sobolevskii]. Muitos dos nossos resultados podem ser pensados como adaptações das estimativas de Sobolevskii quando substituímos as potências fracionárias de $A(t)$ pelos espaços intermediários $\left(X_{0}, X_{1}\right)_{\alpha, \infty ; K}$ ou $\left(X_{0}, X_{1}\right)_{\alpha, \infty ; K}^{0}$.

Em [19], há análise do caso em que $X_{1}$ não é necessariamente denso em $X_{0}$. Mas ele tem em vista a hipótese de regularidade maximal em que $u^{\prime}$ é regular em todo intervalo de existência o que não é o nosso caso e também trabalha com os espaço $\left(X_{0}, X_{1}\right)_{\alpha, 1 ; \infty}$ e $\left(X_{0}, X_{1}\right)_{\alpha, 1 ; \infty}^{o} \operatorname{com} A(t)=A$, constante. Ainda assim não deixa de ser uma boa referência.

Teorema 4.0.12 Suponha $A \in \mathcal{A}, \mathcal{A}$ um conjunto regular como em 3.1.1, mas substitua $[0, T]$ por $[\tau, \tau+T]$. Suponha também $f \in C^{\rho}\left([\tau, \tau+T], X_{0}\right)$. Seja $\beta \in(0,1]$. Chame $X_{\beta}:=\left(X_{0}, X_{1}\right)_{\beta, \infty ; K}$ e $X_{\beta}^{0}=\left(X_{0}, X_{1}\right)_{\beta, \infty ; K}^{o}$. Então se $x(t)$ é a solução do problema $d x / d t+A(t) x=f(t), t \in(\tau, \tau+T], x(\tau)=\xi$, valem as seguintes afirmações, em que todas constantes são uniformes em $A \in \mathcal{A}$,

1. Se $0 \leq \alpha<\beta$ e $\xi \in X_{\beta}$, então $x \in C^{\eta}\left([\tau, \tau+T], X_{\alpha}\right)$, onde $\eta \in(0, \beta-\alpha)$. Além disso, se $c(\alpha, \beta)$ e $c(\alpha)$ denotam a dependência das constantes,

$$
\|x(t+h)-x(t)\|_{X_{\alpha}} \leq C|h|^{\beta-\alpha-\delta}
$$


onde $C=c(\alpha, \beta)|h|^{\delta}\|\xi\|_{\beta}+c(\alpha)|h|^{\delta}(\ln |h|+1) \max _{s \in[\tau, \tau+T]}\|f(s)\|_{X_{0}}$. Então $C=O\left(|h|^{\delta}\right)$ qualquer $\delta \in(0, \beta-\alpha)$.

2. para $t \in[\tau, \tau+T]$, e se $\xi \in X_{\beta}^{\circ}$,

$$
\begin{aligned}
& \|A(t) x(t)\|_{X_{0}} \\
& \quad \leq c\left(\|f(t)\|_{X_{0}}\right. \\
& \left.\quad+\max _{t, s \in[\tau, \tau+T]} \frac{\|f(t)-f(s)\|_{X_{0}}}{|t-s|^{\rho}}\right)+c(\beta) o(1)|t-\tau|^{\beta-1}\|\xi\|_{X_{\beta}}
\end{aligned}
$$

com o(1) se $t \rightarrow \tau$. Não há o(1) se somente $\xi \in X_{\beta}$.

3. A mesma estimativa do item anterior para $\|x(t)\|_{X_{1}}$.

4. A função $t \rightarrow d x(t) / d t:(\tau, \tau+T] \rightarrow X_{\gamma}, \gamma \in[0, \min \{\rho, \epsilon\})$, com

$\left\|\frac{d x}{d t}(t)\right\|_{X_{\gamma}} \leq c(\beta, \gamma)|t-\tau|^{\beta-\gamma-1}\left\{|t-\tau|^{1-\beta}\left(\|f(t)\|_{X_{0}}+\max _{s[\text { tau }, t]} \frac{\|f(t)-f(s)\|}{|t-s|^{p}}\right)+\|\xi\|_{X_{\beta}}\right\}$.

5. a função $t \rightarrow d x(t) / d t:\left[\tau^{+}, \tau+T\right] \rightarrow X_{\theta}$ pertence à $C^{\gamma-\theta}\left(\left[\tau^{+}, \tau+T\right], X_{\theta}\right)$ para $\tau^{+} \in(\tau, \tau+T]$ e $\gamma \in(0, \min \{\rho, \epsilon\})$ e $\theta \in[0, \gamma)$ e

$$
\begin{aligned}
& \left\|\frac{d x}{d t}(t+h)-\frac{d x}{d t}(t)\right\|_{X_{\theta}} \\
& \quad \leq c(\gamma)|h|^{\gamma-\theta}|t-\tau|^{-\gamma}\left(\|f(t)\|_{X_{0}}+\max _{s \in[\tau, \tau+T]} \frac{\|f(t)-f(s)\|}{|t-s|^{\rho}}\right) \\
& \quad+c(\theta)|h|^{\theta}|t-\tau|^{\beta-\theta-1} .
\end{aligned}
$$

Observação 4.0.13 As partes 1,2 vão ser usadas na aplicação do teorema do ponto fixo à equação parabólica quasilinear. A regularidade da derivada serve para demonstrar existência de soluçôes clássicas na aplicação da teoria abstrata a equações parabólicas, digamos, da Física clássica.

Observação 4.0.14 As estimativas de Sobolevskii em [1] vão ser necessárias em varios momentos da demonstração. Conforme comentada no início do capítulo, Sobolevskii descreveu estimativas para o operador de evolução envolvendo potências fracionárias. Embòra nós trabalhalhemos com espaços intermediários, há casos em que seus cálculos podem ser aproveitados sem necessidade de adaptação do argumento para espaços intermediários. Por exemplo, estimativas do tipo $\left\|A(t)^{\alpha} f\right\| \leq C\|f\|_{X_{0}} \alpha \in[0,1]$ podem ser aproveitadas, 
pois, por 1.3.1, por 1.2.20 e por 1.2.27, $X^{\alpha}(A(t)) \subset\left(X_{0}, X_{1}\right)_{\alpha, \infty ; K}$ com constante de imersão denotada por $c^{\prime}$ e, então, $\|f\|_{\left(X_{0}, X_{1}\right)_{\alpha, 1 ; K}} \leq c^{\prime} C\|f\|_{X_{0}}$.

As estimativas de Sobolevskii utilizadas neste capitulo estão reunidas no lema abaixo.

Prova. Chame de $U$ o operador de evolução para

$$
x(t)=U(t, \tau) \xi+\int_{\tau}^{t} U(t, s) f(s) d s .
$$

A estimatva $\|(U(t+h, \tau)-U(t, \tau)) \xi\| \leq c(\alpha, \beta) h^{\beta-\alpha}$ é um caso particular de 3.4.8. Juntamente com 4.0.15, obtém-se a prova do item 1.

No item 2, é necessário avaliar $A(t) x(t)$. A parcela $A(t) U(t, \tau) \xi$ foi avaliada em 3.4.6 ou em 3.4.3 se somente $\xi \in X_{\beta}$. A conclusão da prova segue da avaliação da parcela remanescente. $O$ teorema 3.1.6 fornece também

$$
\frac{\partial}{\partial t} \int_{\tau}^{t} U(t, s) f(s) d s=f(t)-A(t) \int_{\tau}^{t} U(t, s) f(s) d s .
$$

Com o lema 4.0.15,

$$
\left\|A(t) \int_{\tau}^{t} U(t, s) f(s) d s\right\| \leq c\left(\|f(t)\|+\max _{\tau \leq s \leq t} \frac{\|f(t)-f(s)\|}{\left.|t-s|^{\rho}\right)}\right) .
$$

Uniformemente em $\mathcal{A}, D(A) \subset X_{1}$. Chame de $c^{1}$ a constante de imersão. Então $\|f\|_{X_{1}} \leq\left(c^{1}+\left\|A^{-1}\right\|\right)\|A f\|$ e, segue o item 3 , pois $\left\|A^{-1}\right\| \leq C_{\mathcal{A}}$.

O teorema 3.1 .4 fornece $\frac{\partial}{\partial t} U(t, \tau) \xi=-A(t) U(t, \tau) \xi$ sempre que $t>\tau$. Então

$$
\frac{d x}{d t}(t)=-A(t) U(t, \tau) \xi+\frac{\partial}{\partial t} \int_{\tau}^{t} U(t, s) f(s) d s
$$

Aplicação de 3.4 .3 e 4.0.15 implicam a afirmação do item 4.

Lema 4.0.15 Suponha $A \in \mathcal{A}, \mathcal{A}$ um conjunto regular de $C^{\epsilon}\left([0, T], \mathcal{H}\left(X_{1}, X_{0}\right)\right.$. E chame $X_{\alpha}=\left(X_{0}, X_{1}\right)_{\alpha, \infty ; K}$.

Então, com constantes uniformes para $A \in \mathcal{A}$,

1. quando $\alpha \in[0,1)$

$$
\begin{aligned}
& \left\|\int_{\tau}^{t+h} U_{A}(t+h, s) f(s) d s-\int_{\tau}^{t} U_{A}(t, s) f(s) d s\right\|_{X_{\alpha}} \\
& \quad \leq c(\alpha) h^{1-\alpha}(|\ln h|+1) \max _{\tau \leq s \leq t+h}\|f(s)\| .
\end{aligned}
$$


2. quando $\alpha \in[0, \min \{\epsilon, \rho\})$,

$$
\left\|\frac{\partial}{\partial t} \int_{\tau}^{t} U_{A}(t, s) f(s) d s\right\|_{X_{\alpha}} \leq c(\alpha)|t-\tau|^{-\alpha}\left(\|f(t)\|+\max _{\tau \leq s \leq t} \frac{\|f(t)-f(s)\|}{\left.|t-s|^{\rho}\right)}\right) .
$$

3. quando $0 \leq \alpha \leq \gamma<\min \{\epsilon, \rho\}$,

$$
\begin{aligned}
& \left\|\frac{\partial}{\partial t} \int_{\tau}^{t+h} U_{A}(t+h, s) f(s) d s-\frac{\partial}{\partial t} \int_{\tau}^{t} U_{A}(t, s) f(s) d s\right\|_{X_{\alpha}} \\
& \quad \leq c(\gamma)(t-\tau)^{-\gamma} h^{\gamma-\alpha}\left(\|f(t)\|+\max _{\tau \leq s \leq t} \frac{\|f(t)-f(s)\|}{|t-s|^{\rho}}\right. \\
& \left.\quad+\max _{t \leq s \leq t+h} \frac{\|f(t+h)-f(s)\|}{|t+h-s|^{\rho}}\right)
\end{aligned}
$$

Prova. Todas consequências da imersão $X^{\alpha}(A(t)) \subset X^{\alpha}$ e das estimativas de [Sobolevskii[1]] com a observação de que todas dependem somente das constantes associadas ao conjunto regular $\mathcal{A}$. O comentário em [Sobolevskii[1], página 49] também corrobora isto. Especificamente, para 1, [[1], página 33, (2.9)]. Para 2, [[1], página 34, (2.11)]. Para 3, [[1], página 40, (2.27)].

Teorema 4.0.16 Suponha $A, B \in \mathcal{A}, \mathcal{A}$ como em 3.1.1, mas substitua o intervalo $[0, T]$ por $[\tau, \tau+T]$. Suponha também $f, g \in C^{\rho}\left([\tau, \tau+T], X_{0}\right)$. E se $X_{\beta}:=\left(X_{0}, X_{1}\right)_{\beta, \infty ; K}$, seja $x_{0}, y_{0} \in X_{\beta} \operatorname{com} \beta \in(0,1]$.

Então, se $x$ e y são soluções das equações $d x / d t+A(t) x=f(t), d y / d t+$ $B(t) y=g(t), t \in\left(\tau, \tau+T\right.$ e $x(\tau)=x_{0}, y(\tau)=y_{0}$ ent $\tilde{a} o$

$$
\begin{aligned}
y(t) & -x(t) \\
& =U_{A}(t, \tau)\left(y_{0}-x_{0}\right)+\int_{\tau}^{t} U_{A}(t, s)(A(s)-B(s)) y(s) d s \\
& +\int_{\tau}^{t} U_{A}(t, s)(g(s)-f(s)) d s .
\end{aligned}
$$

Prova. A diferença das equações fornece

$$
\frac{d}{d t}(y(t)-x(t))+A(t)(y(t)-x(t))=(A(t)-B(t)) y(t)+g(t)-f(t) .
$$

A termo $\|(A(t)-B(t)) y(t)\| \leq\|(A(t)-B(t))\|_{L\left(X_{1}, X_{0}\right)}\|y(t)\|_{X_{1}}$, pelo teorema 4.0.12, tem majorante de ordem $O\left(|t-\tau|^{\beta-1}\right)$. A conclusão segue do teorema 3.1.6. 
Observação 4.0.17 Um caminho opcional é provar que o termo mencionado é uma função Hölder contínua em $\left[\tau^{+}, T\right]$, a partir de qualquer $\tau^{+} \in(\tau, T]$. Com isto a equação integral vale para $\tau^{+}$no lugar $\tau$. Então toma-se o limite quando $\tau+\rightarrow \tau$. Assim é feito por [Sobolevskii[1], página 51]. 


\section{Capítulo 5}

\section{Equação de Evolução Parabólica}

Nosso objetivo é descrever uma maneira de usar os resultados dos capítulos precedentes para demonstrar existência e regularidade de solução para a equação parabólica quasilinear.

Por equação parabólica quasilinear em um espaço de Banach $X_{0}$, entendemos uma equação com a forma

$$
\frac{d u}{d t}+A(t, u) u=f(t, u), \tau<t, u(\tau)=\xi .
$$

A hipótese básica é que $D(A(t, u))=X_{1}$, constante, $X_{1} \subset X_{0}$, densa e continuamente e $A(t, u)$ pertence a $\mathcal{H}\left(X_{1}, X_{0}\right), \mathcal{H}\left(X_{1}, X_{0}\right)$ com em 1.2.13, quando $(t, u)$ estão em um aberto de $\mathbb{R} \times X_{\alpha}$. Aqui $X_{\alpha}:=\left(X_{0}, X_{1}\right)_{\alpha, \infty ; K}^{o}$.

Para poder enunciar as hipóteses,, vamos demonstrar um simples resultado de topologia. O resultado provoca alguma estranhesa. Ele, no entanto, possibilita encontrar solução para equação quasilinear no mesmo espaço de fase do dado inicial. Isto não ocorria com o teorema corresponde de Sobolevskii [[1], teorema 7, página 7]. Em outras palavras, era suposto dado inicial mais regular em $\xi \in X_{\beta}$ para solução em $u(t) \in C\left([0, T], X_{\alpha}\right)$ com $\alpha<\beta$, sem continuidade em $X_{\beta}$. Na verdade, Sobolevskii usava espaços de potência $X^{\alpha}$. O método, entretanto, já podia ter sido usado por Sobolevskii.

A heurística está bem descrita no livro de Amann [[5], página xxviii]. Aparentemente, o método foi usado originalmente em [Amann[4]]. Há uma lacuna no trabalho de Amann na minha opinião. No artigo [[4]], ele desenvolve a sua teoria de existência para a equação quasilinear parabólica. 
Entretanto, na ocasião, ele não possuia estimativas do tipo de Sobolevskii para espaços intermediários ou de interpolação. Então ele as assume como hipótese. Assim entendemos seu teorema onde apenas enuncia que as estimativas de Sobolevskii se mantém para espaços intermediários ou de interpolação. Em seu livro [[5]], as estimativas de Sobolevskii para espaços de interpolação começam a ser descritas. Mas não está claro que toda a lacuna foi preenchida.

A restrição de Sobolevskii continua bem aceita, embora o método de Amann funcione. Em [Lunardi[15], página 335] lê-se "the advantage of this approach (referência ao trabalho de Sobolevskii, Amann sobre equação quasilinear parabólica) is threefold: first, one can allow inicial data not belonging to $D\left(X_{1}\right.$ aqui), but to an intermediate space between $X_{\theta}$ and $D$ (however, not to $\left.X_{\theta}\right)$ ".

Observação 5.0.18 Neste capítulo, $X_{\alpha}=\left(X_{0}, X_{1}\right)_{\alpha, \infty ; K}^{o}$.

\subsection{Topologia}

Proposição 5.1.1 Suponha $X_{\alpha} \subset X_{\beta}$. Se $U \subset \mathbb{R} \times X_{\beta}$ é aberto então $U \cap\left(\mathbb{R} \times X_{\alpha}\right)$ é um aberto de $\mathbb{R} \times X_{\alpha}$. E se $U \subset \mathbb{R} \times X_{\alpha}$ é um aberto $e$ se $V \subset \mathbb{R} \times X_{\beta}$ é aberto com $V \supset U$ então $V \cap\left(\mathbb{R} \times X_{\alpha}\right)$ é um aberto de $\mathbb{R} \times X_{\alpha}$ que contém $U$.

Prova. Suponha $\left(t_{0}, u_{0}\right) \in U \cap\left(\mathbb{R} \times X_{\alpha}\right)$. Pela hipótese da primeira afirmação, é possível achar $r_{0}>0$ para que se $\left|t-t_{0}\right|<r_{0}$ e $\left\|u-u_{0}\right\|_{X_{\beta}}<r_{0}$, vizinhança quadrada de $\left(t_{0}, u_{0}\right)$, então $(t, u) \in U$. Portanto a vizinhança de $\left(t_{0}, u_{0}\right)$ em $\mathbb{R} \times X_{\alpha}$ definida pelos $(t, u)$ que obedecem

$$
\left|t-t_{0}\right|<r_{0},\left\|u-u_{0}\right\|_{X_{\alpha}}<r_{0} / a
$$

com $a$ a constante de imersão de $X_{\alpha} \subset X_{\beta}$, está também em $U$. Logo $U \cap\left(\mathbb{R} \times X_{\alpha}\right)$ é aberto em $\mathbb{R} \times X_{\alpha}$.

Adicionalmente, é necessário notar que se $u$ estáem $X_{\alpha}$ então $t^{-\alpha} K\left(t, f, X_{0}, X_{1}\right) \rightarrow$ 0 se $t \rightarrow 0+\mathrm{e}$, por maior razão, $t^{-\beta} K\left(t, f, X_{0}, X_{1}\right) \rightarrow 0$ se $t \rightarrow 0+$ quando $\alpha>\beta$.

Na segunda afirmação, o fato de que $V \cap\left(\mathbb{R} \times X_{\alpha}\right)$ é aberto segue diretamente da primeira parte. E também, por hipótese, $V$ contém uma parte $U$, que está em $\mathbb{R} \times X_{\alpha}$, então, obviamente, sua interseção com $\mathbb{R} \times X_{\alpha}$ a incluirá. 
Observação 5.1.2 Mais tarde vai ficar claro o porquê da inclusão da afirmação final. Mas só para adiantar, parte das hipóteses da equação quasilinear parabólica são formuladas em relação a $U \subset \mathbb{R} \times X_{\beta}$ para garantir solução em $U \cap\left(\mathbb{R} \times X_{\alpha}\right), \alpha>\beta$. Então a proposição diz que não precisamos depender da bondade(sic) de $U$.

\subsection{Mais cálculo em espaço de Banach}

Teorema 5.2.1 Suponha $X, Y$ espaços de Banach, $U \subset X$ aberto e $J \subset \mathbb{R}$ um intervalo limitado. Suponha $r$ um inteiro positivo. Para todo $k, 0 \leq k \leq$ $r$, suponha que a função

$$
F^{(k)}: J \times U \rightarrow L_{\text {sym }}^{k}(X, Y),
$$

onde $F^{(k)}(t, x)=\partial^{k} F(t, x) / \partial^{k} x$, é uniformemente Hölder ou Lipschitz contínua em $t$ e localmente Hölder ou Lipschitz contínua em x. Precisamente, existem números $\rho_{1}, \rho_{2} \in(0,1]$ para

$$
\left\|F^{(k)}\left(t_{2}, x_{2}\right)-F^{(k)}\left(t_{1}, x_{1}\right)\right\|_{L_{s y m}^{k}(X, Y)} \leq L\left(\left|t_{2}-t_{1}\right|^{\rho_{1}}+\left\|x_{2}-x_{1}\right\|_{X}^{\rho_{2}}\right)
$$

se $x_{1}, x_{2} \in$ viz $\left(x_{0}\right) \subset U$ para cada $x_{0} \in U$, todo $k, 0 \leq k \leq r$ e $t_{1}, t_{2} \in J$.

Então a função composição

$$
x \rightarrow F(\cdot, x(\cdot)): C^{\alpha}(J, U) \rightarrow C^{\beta}(J, Y)
$$

é $C^{r}$ se $\beta \geq 0$ e $\beta<\min \left\{\rho_{1}, \alpha \rho_{2}\right\}$. Mais ainda, $F$ é $C^{r, \rho} \operatorname{com} \rho=\phi \rho_{2} e$ $\phi$ um número que obedece $(1-\phi) \min \left\{\rho_{1}, \alpha \rho_{2}\right\} \geq \beta$. Suponha as hipóteses sobre $F$ para todo $r$ e se a série para $x \rightarrow F(t, x)$ converge uniformemente em $t \in J$ perto de cada $x \in U$, então a função composição é analítica.

Prova. A prova é uma aplicação do recíproco do teorema de Taylor [[8], página 12]. Há também uma excelente demonstração deste teorema em [[13]].

Suponha $x_{0} \in C^{\alpha}(J, U)$ e denote por $x \in C^{\alpha}(J, U)$ e $h \in C^{\alpha}(J, X)$ com $x+h \in C^{\alpha}(J, U)$. Sempre possível se $(x, h)$ está em uma vizinhança de $\left(x_{0}, 0\right)$ em $C^{\alpha}(J, U) \times C^{\alpha}(J, X)$.

Para cada $t \in J$, por hipótese, a função $x \rightarrow F(t, x): U \rightarrow Y$ é $C^{k} \mathrm{e}$ portanto, pelo teorema de Taylor,

$$
\begin{aligned}
F(t & , x(t)+h(t))-\sum_{k=0}^{r} F^{(k)}(t, x(t))\left[h^{k}(t)\right] \\
& =\int_{0}^{1} \frac{(1-\theta)^{r-1}}{(r-1) !}\left(F^{(r)}(t, x(t)+\theta h(t))-F^{(r)}(t, x(t))\right)\left[h^{r}(t)\right] d \theta .
\end{aligned}
$$


Para concluir prova da afirmação inicial, resta verificar se, uniformemente em $\theta$,

$$
\left\|F^{(r)}(\cdot, x(\cdot)+\theta h(\cdot))-F^{(r)}(\cdot, x(\cdot))\right\|_{L_{s y m}^{r}\left(C^{\alpha}(J, X), C^{\beta}(J, Y)\right)} \rightarrow 0
$$

se $(x, h) \rightarrow\left(x_{0}, 0\right)$ em $C^{\alpha}(J, U) \times C^{\alpha}(J, X)$.

Explicitanto mais as normas

$$
\begin{aligned}
& \left\|F^{(r)}(\cdot, x(\cdot)+\theta h(\cdot))-F^{(r)}(\cdot, x(\cdot))\right\|_{L_{s y m}^{r}\left(C^{\alpha}(J, X), C^{\beta}(J, Y)\right)} \\
& \quad \leq \sup _{s \in J}\left\|F^{(r)}(s, x(s)+\theta h(s))-F^{(r)}(s, x(s))\right\|_{L_{s y m}^{r}(X, Y)}+ \\
& \quad \sup _{t, s \in J, t \neq s} \frac{1}{|t-s|^{\beta}}\left\|F^{(r)}(t, x(t)+\theta h(t))-F^{(r)}(s, x(s)+\theta x(s))\right\|
\end{aligned}
$$

A primeira parcela não excede $L\|h\|_{C([0, T], X)}^{\rho_{2}}$. A avaliação da outra é menos direta, sendo necessário lançar mão de duas desigualdades elementares que passo a descrever. Se $a, b \geq 0$ então $(a+b)^{\phi} \leq a^{\phi}+b^{\phi}$ e $\min \{a, b\} \leq a^{\phi} b^{1-\phi}$ sempre que $\phi$ é um número em $[0,1]$. Com isto, entendendo o sup em $\{t, s \in$ $J, t \neq s\}$

$$
\begin{aligned}
& \sup \frac{1}{|t-s|^{\beta}}\left\|F^{(r)}(t, x(t)+\theta h(t))-F^{(r)}(s, x(s)+\theta h(s))+F^{(r)}(t, x(t))-F^{(r)}(s, x(s))\right\|_{L} \\
& \leq \sup \frac{1}{|t-s|^{\beta}}\left\{\min \left\{2 \theta\|h\|_{C(J, X)}, 2 L|t-s|^{\rho_{1}}+2 L\|x(t)-x(s)\|_{X}^{\rho_{2}}+L\|h(t)-h(s)\|_{X}^{\rho_{2}}\right\}\right\} .
\end{aligned}
$$

Se, digamos, $\left\|x-x_{0}\right\|_{C^{\alpha}(J, X)} \leq r$, então $\|x(t)-x(s)\|_{X} \leq\left(r+a_{0}\right)|t-s|^{\alpha}$ com $a_{0}$ para $\left\|x_{0}(t)-x_{0}(s)\right\|_{X} \leq a_{0}|t-s|^{\alpha}$. E também se $\|h\|_{C^{\alpha}(J, X)} \leq r$ então $\|h(t)-h(s)\|_{X} \leq r|t-s|^{\alpha}$. Portanto, se $\mu=\min \left\{\rho_{1}, \alpha \rho_{2}\right\}$, o último majorante não excede

$$
c\|h\|_{C^{\alpha}(J, X)}^{\phi} \sup \left\{|t-s|^{(1-\phi) \mu} /|t-s|^{\beta}\right\}
$$

$\operatorname{com} c=\max \left\{2,2 L\left(|t-s|^{\rho_{1}-\mu}+\left(r+a_{0}\right)^{\rho_{2}}|t-s|^{\alpha \rho_{2}-\mu}+r^{\rho_{2}}|t-s|^{\alpha \rho_{2}-\mu}\right)\right\}$. Como, por hipótese, $(1-\phi) \mu-\beta \geq 0$ e por $J$ um intervalo limitado,

$$
\begin{aligned}
& \left\|F^{(r)}(\cdot, x(\cdot)+\theta h(\cdot))-F^{(r)}(\cdot, x(\cdot))\right\|_{L_{s y m}^{r}\left(C^{\alpha}(J, X), C^{\beta}(J, Y)\right)} \\
& \quad=O\left(\|h\|_{C^{\alpha}(J, X)}^{\phi}\right) .
\end{aligned}
$$

E também, seguindo um raciocínio análogo, obtém-se também $F^{(k)}(\cdot, x(\cdot) \in$ $L_{\text {sym }}^{k}\left(C^{\alpha}(J, X), C^{\beta}(J, Y)\right)$ para $0 \leq k \leq r$. Portanto, está completada a prova não só a da primeira, mas de ambas as afirmações sobre diferenciabilidade. Para a análise da analiticidade, estimativa do resto de Taylor prova o resultado. 


\subsection{Teorema de existência e unicidade}

Teorema 5.3.1 Chame $X_{\gamma}=\left(X_{0}, X_{1}\right)_{\gamma, \infty ; K}^{o}$ para $\gamma \in[0,1)$. Suponha $\alpha>$ $\beta, \alpha, \beta \in[0,1)$ e $U \subset \mathbb{R} \times X_{\beta}$ aberto. Sejam $\lambda \in \Lambda$ e $\mu \in \Upsilon, \Lambda$ e $\Upsilon$ abertos de espaços de Banach. Suponha

$$
(t, x) \rightarrow f(t, x, \lambda): U \rightarrow X_{0}
$$

$e$

$$
(t, x) \rightarrow A(t, x, \mu): U \rightarrow \mathcal{H}\left(X_{1}, X_{0}\right)
$$

funções localmente Hölder contínuas em $t$ e locamente Lipschitz contínuas em $x$ para cada $\lambda$ e $\mu$. Precisamente, para cada $\lambda_{1}$ e $\mu_{1}$, e para cada ponto $\left(t_{1}, x_{1}\right) \in U$, exite uma vizinhança $V$ em $\mathbb{R} \times X_{\beta}$ tal que todo $(t, x) \in V e$ $(s, y) \in V$,

$$
\begin{gathered}
\left\|f\left(t, x, \lambda_{1}\right)-f\left(s, y, \lambda_{1}\right)\right\|_{X_{0}} \leq L\left(|t-s|^{\rho}+\|x-y\|_{X_{\beta}}\right), \\
\left\|A\left(t, x, \mu_{1}\right)-A\left(s, y, \mu_{1}\right)\right\|_{L\left(X_{1}, X_{0}\right)} \leq L\left(|t-s|^{\epsilon}+\|x-y\|_{X_{\beta}}\right),
\end{gathered}
$$

para alguma constante $L>0, \epsilon>0$ e $\rho>0$.

Então para cada $\left(t_{0}, u_{0}, \lambda_{0}, \mu_{0}\right) \in U \cap\left(\mathbb{R} \times X_{\alpha}\right) \times \Lambda \times \Upsilon$ existe $T>0 e$ uma um única função u que satisfaz à equação no espaço $X_{0}$

$$
d u / d t+A\left(t, u(t), \mu_{0}\right) u=f\left(t, u(t), \lambda_{0}\right)
$$

se $t>t_{0}$ e $u\left(t_{0}\right)=u_{0}$. A função u pertence à $C\left(\left[t_{0}, t_{0}+T\right], X_{\alpha}\right) \cap C^{1}\left(\left(t_{0}, t_{0}+\right.\right.$ $\left.T], X_{0}\right)$ e $u(t) \in X_{1}$ se $t \in\left(t_{0}, t_{0}+T\right]$.

Prova. Como $\lambda_{0}$ e $\mu_{0}$ estão fixos, por conveniência, denotamos só $f(t, u(t))$ em vez de $f(t, u(t), \lambda)$ e procedemos igualmente com $A(t, u(t), \mu)$.

Sem perda de generalidade, suponha $t_{0}=0$. Senão, com a mudança de variável $t \rightarrow t+t_{0}$, a equação recai neste caso. Explicitamente, chamando $s=t-t_{0}, v(s)=u\left(s+t_{0}\right), \bar{f}(s, u)=f\left(s+t_{0}, u\right)$ e $\bar{A}(s, u)=A\left(s+t_{0}, u\right)$, a equação em $v$ é

$$
d v / d t(s)+\bar{A}(s, v(s)) v(s)=\bar{f}(s, v(s)), v(0)=u_{0} .
$$

Por hipótese, em uma viz $\left(t_{0}, u_{0}\right) \subset U \subset \mathbb{R} \times X_{\beta},\left\|f\left(t^{2}, u^{2}\right)-f\left(t^{1}, u^{1}\right)\right\|_{X_{0}} \leq$ $L\left(\left|t^{2}-t^{1}\right|+\left\|u^{2}-u^{1}\right\|_{X_{\beta}}\right)$. Então também, em uma vizinhança de $\overline{v i z}\left(0, u_{0}\right) \subset$ 
$\mathbb{R} \times X_{\beta}$, em que $(s, u) \in \overline{v i z}\left(0, u_{0}\right)$ se $\left(s+t_{0}, u\right) \in v i z\left(t_{0}, u_{0}\right)$, temos idêntica regularidade para $\bar{f}$, pois $\left\|\bar{f}\left(s^{2}, u^{2}\right)-\bar{f}\left(s^{1}, u^{1}\right)\right\|_{X_{0}} \leq L\left(\left|s^{2}-s^{1}\right|+\left\|u^{2}-u^{1}\right\|_{X_{\beta}}\right)$. $\mathrm{E}$, com argumento idêntico, obtêm-se a mesma regularidade para $\bar{A}$.

Admita então que $t_{0}=0$. A partir daqui, começa a preparação para aplicação do teorema de ponto fixo de Banach.

Seja $T>0$. Chame $\eta$ um número em $(0, \alpha-\beta)$,

$$
W(T)=C\left([0, T], X_{\alpha}\right) \cap C^{\eta}\left([0, T], X_{\beta}\right),
$$

e defina $S(T)=\left\{w \in W(T),\|w(t)-w(s)\|_{X_{\beta}} \leq k|t-s|^{\eta},\right\}$. O espaço $S(T)$ com a norma de $C\left([0, T], X_{\alpha}\right)$ é um espaço métrico completo, pois $C\left([0, T], X_{\alpha}\right) \subset C\left([0, T], X_{\beta}\right)$ continuamente.

Por hipótese em uma vizinhança de $\left(0, u_{0}\right)$, digamos, com $\sigma>0$ e $\delta>0$,

$$
V=\left\{(t, w) \in \mathbb{R} \times X_{\beta}, 0 \leq t \leq \sigma,\left\|w-u_{0}\right\|_{X_{\beta}} \leq \delta\right\},
$$

$V \subset U$, em cujos pontos $\left(t^{1}, w^{1}\right),\left(t^{2}, w^{2}\right) \in V, f$ e $A$ obedecem

$$
\begin{gathered}
\left\|f\left(t^{2}, w^{2}\right)-f\left(t^{1}, w^{1}\right)\right\|_{X_{0}} \leq L\left(\left|t^{2}-t^{1}\right|^{\rho}+\left\|w^{2}-w^{1}\right\|_{X_{\beta}}\right), \\
\left\|A\left(t^{2}, w^{2}\right)-A\left(t^{1}, w^{1}\right)\right\|_{L\left(X_{1}, X_{0}\right)} \leq L\left(\left|t^{2}-t^{1}\right|^{\epsilon}+\left\|w^{2}-w^{1}\right\|_{X_{\beta}}\right),
\end{gathered}
$$

para algum $L>0$.

Supondo $T \leq \sigma$ e $T^{\eta} \leq \delta$ e também, para obter constantes mais simples, suponha $T \leq 1$, então, $S(T) \subset V$ e para todo $w \in S(T)$ e $t, s \in[0, T]$,

$$
\begin{gathered}
\|f(t, w(t))-f(s, w(s))\|_{X_{0}} \leq L(1+k)|t-s|^{\mu}, \\
\|A(t, w(t))-A(s, w(s))\|_{L\left(X_{1}, X_{0}\right)} \leq L(1 L+k)|t-s|^{\mu},
\end{gathered}
$$

em que $\mu=\min \{\rho, \epsilon, \eta\}$.

Em particular, $A\left(u_{0}\right), A\left(u_{0}\right)(t)=A\left(t, u_{0}\right)$, pertence à $C^{\mu}\left([0, T], \mathcal{H}\left(X_{1}, X_{0}\right)\right)$.

Seja $\mu^{-} \in(0, \mu)$. Obviamente $A\left(u_{0}\right) \in C^{\mu^{-}}\left([0, T], \mathcal{H}\left(X_{1}, X_{0}\right)\right)$. Então seja $B\left(A\left(u_{0}\right), r\right), r>0$, a bola de centro $u_{0}$ e raio $r$ em $C^{\mu^{-}}\left([0, T], \mathcal{H}\left(X_{1}, X_{0}\right)\right)$ como descrita em 3.3.1. Portanto se também $L(1+k) T^{\eta}+2 T^{\mu-\mu^{-}}<r$, o operador $A(w), A(w)(t)=A(t, w(t))$, pertence à $B\left(u_{0}, r\right)$ para todo $w \in$ $S(T)$. 
Portanto $A(w) \in \mathcal{A}$ e o operador de evolução $U_{A(w)}$ está bem definido quando $w \in S(T)$. Então seja

$$
G(w):=U_{A(w)}(t, 0) u_{0}+\int_{0}^{t} U_{A(w)}(t, s) f(s, w(s)) d s .
$$

Veriquemos se a função $G$ define uma contração em $S(T)$.

Pela 3.3.9, $G(w) \in C\left([0, T], X_{\alpha}\right)$ quando $w \in S(T)$. E também por 4.0.12, $G(w) \in C^{\eta}\left([0, T], X_{\beta}\right)$ com

$$
\|G(w)(t)-G(w)(s)\|_{X_{\beta}} \leq k|t-s|^{\eta}
$$

se $T$ for escolhido pequeno o suficiente. Note que $\|f(t, w)\|_{X_{0}} \leq L(1 L+$ $k) T^{\mu}+\left\|f\left(0, x_{0}\right)\right\|_{X_{0}}$ se $t \in[0, T]$ e $w \in S(T)$.

Portanto para $T$ pequeno o suficiente $G$ com domínio $S(T)$ tem imagem em $S(T)$.

Se $w^{1}, w^{2} \in S(T)$, então $x^{1}=G\left(w^{1}\right)$ é solução da equação $d x^{1} / d t+$ $A\left(w^{1}\right)(t) x^{1}=f\left(t, w^{1}\right), x^{1}(0)=x_{0}$ em virturde das propriedades do operador de evolução. Raciocínio análogo para $G\left(w^{2}\right)$. Então, aplicando 4.0.16,

$$
\begin{aligned}
& \left\|\left(G\left(w^{2}\right)-G\left(w^{1}\right)\right)(t)\right\|_{X_{\alpha}} \\
& \quad \leq \int_{0}^{t}\left\|U_{A\left(w^{1}\right)(t, s)}\right\|_{L\left(X_{0}, X_{\alpha}\right)}\left\|\left(A\left(w^{1}\right)(s)-A\left(w^{2}\right)(s)\right)\right\|_{L\left(X_{1}, X_{0}\right)}\left\|G\left(w^{2}\right)(s)\right\|_{X_{1}} d s \\
& \quad+\int_{0}^{t}\left\|U_{A\left(w^{1}\right)}(t, s)\right\|_{L\left(X_{0}, X_{\alpha}\right)}\left\|f\left(s, w^{2}(s)\right)-f\left(s, w^{1}(s)\right)\right\|_{X_{0}} d s
\end{aligned}
$$

Com isto, como $\left\|U_{A\left(w^{1}\right)}(t, s)\right\|_{L\left(X_{0}, X_{\alpha}\right)} \leq c(\alpha)(t-s)^{-\alpha}$ e $\left\|w^{2}(s)\right\|_{X_{1}} \leq$ $c(\alpha) s^{-\alpha} o(1)$, e pelo fato de que $X_{\alpha} \subset X_{\beta}$,

$\left\|G\left(w^{2}\right)-G\left(w^{1}\right)\right\|_{C\left([0, T], X_{\alpha}\right)} \leq L c(\alpha, \beta) o(1)\left\|w^{2}-w^{1}\right\|_{C\left([0, T], X_{\alpha}\right)}+\frac{T^{1-\alpha}}{1-\alpha}\left\|w^{2}-w^{1}\right\|_{\left.C[0, T], X_{\alpha}\right)}$

Então se $T$ for pequeno o suficiente,

$$
\left\|G\left(w^{2}\right)-G\left(w^{1}\right)\right\|_{C\left([0, T], X_{\alpha}\right)} \leq a\left\|w^{2}-w^{1}\right\|_{\left.C[0, T], X_{\alpha}\right)}
$$

com $0<a<1$ para todo $w \in S(T)$. Então o teorema da Contração de Banach aplica-se.

Observação 5.3.2 A condição de que as estimativa para o resolvente de $A(t, x)$ seja em $\{\mathbb{R e} \lambda \geq 0\}$ não é restritiva. Se elas valem em, digamos, $\{\mathbb{R e} \lambda \geq \omega\}$, fazemos a mudança $A(t, x) \rightarrow A(t, x)+w I$ e $f(t, x) \rightarrow f(t, x)+$ $w x$. 
Observação 5.3.3 No caso semilinear, $A(t, x)=A(t)$, o teorema vale também se $\alpha=\beta$. É necessário adaptar a demonstração de [[8], teorema 3.3.3, página 54], pois aqui há dependência com t. Mas dispomos de estimativas para fazer a substituiçáa do cálculo com semigrupo para o cálculo com operador de evolução, apresentadas na seção 3.4 .

Os resultados de regularidade de Henry também vão valer neste caso, 3.4.4, 3.4.5 e 3.4.5, pois temos resultados de regularidade correspondentes ao lema 3.4.2 para fazer a substituição de semigrupo para operador de evolução, apresentados na seção 3.3 .

Teorema 5.3.4 $O$ conjunto $\{(t, \tau, \xi, \mu, \lambda), t \in I(\tau, \xi, \mu, \lambda)\}$ é aberto em $[\tau, \infty) \times U \cap\left(\mathbb{R} \times X_{\alpha}\right) \times \Upsilon \times \Lambda$ se $I(\tau, \xi, \mu, \lambda)$ é o intervalo maximal da solução de du/dt $+A(t, u, \mu) u=f(t, u, \lambda), t>\tau, u(\tau)=\xi$ e se as hipóteses sobre $A(t, u, \mu)$ e $f(t, u, \lambda)$ valem em uma vizinhança de cada $\left(t_{0}, u_{0}, \mu_{0}, \lambda_{0}\right) \in$ $U \cap\left(X_{\alpha} \times \mathbb{R}\right) \times \Upsilon \times \Lambda$.

$E$ também em uma vizinhança de cada $\left(t_{0}, u_{0}, \mu_{0}, \lambda_{0}\right) \in U \cap\left(\mathbb{R} \times X_{\alpha}\right) \times$ $\Upsilon \times \Lambda$, a função $G$ descrita na prova anterior é uma contração em $S(T)$ para algum $T>0$.

Prova. Devido a regularidade de $A$ e $f$ em uma vizinhança de cada $\left(\tau_{0}, \xi_{0}, \mu_{0}, \lambda_{0}\right)$, existe $\bar{T}$ para que $I(\tau, \xi, \mu, \lambda) \supset[\tau, \tau+\bar{T}]$ se $(\tau, \xi, \mu, \lambda) \in v i z\left(t_{0}, \xi_{0}, \mu_{0}, \lambda_{0}\right)$, pois nesta vizinhança a função $G$ que foi definida na prova anterior mantémse uma contração em $S(\bar{T})$.

Para ver isto é necessário analisar função $G$. Em particular do termo $\|G(w)(t)\|_{X_{1}}$. Seu valor é

$$
G(w)(t)=U_{A(w)}(t, 0) \xi+\int_{0}^{t} U_{A(w)}(t, s) f(s, w(s)) d s .
$$

No cálculo da prova, $\xi=x_{0}$. A norma da segunda parcela em $X_{1}$ é limitada de acordo com a prova de 4.0 .12 , portanto não oferece problemas

A parte delicada consiste na análise da outra parcela. Conforme 4.0.12, $\left\|U_{A(w)}(t, 0) x_{0}\right\| \leq c(\alpha) t^{\alpha-1} o(1)$. Mas como não é possível controlar $o(1)$ quando trocamos $x_{0}$ por um $\xi$, ainda que próximo, não havendo garantia que $G$ se mantém contração na vizinhança de $x_{0}$. Mas $\xi=\xi-x_{0}+x_{0}$ e então

$$
\left\|U_{A(w)}(t, 0) \xi\right\|_{X_{1}} \leq c(\alpha) o(1) t^{\alpha-1}+c(\alpha) t^{\alpha-1}\left\|\xi-x_{0}\right\|_{X_{\alpha}} .
$$

Portanto se $T$ e $\left\|\xi-x_{0}\right\|_{X_{\alpha}}$ forem suficientemente pequenos, $G$ continuará sendo uma contração. 
Se, para $(\tau, \xi, \mu, \lambda) \in v i z\left(t_{0}, \xi_{0}, \mu_{0}, \lambda_{0}\right)$, ocorresse $I(\tau, \xi, \mu, \lambda)=\left[\tau, \tau+T_{1}\right]$, $T_{1}>0$, então aplicando o teorema novamente para $d v / d t+A(t, v) v=f(t, v)$, $t>\tau+T_{1}, v\left(\tau+T_{1}\right)=u\left(\tau+T_{1}\right)$, com $u$ a solução em $\left[\tau, \tau+T_{1}\right]$, obteríamos através de $v$ um prolongamento de $u$ o que contraria a hipótese de $u$ ser maximal.

Observação 5.3.5 O argumento para a contração uniforme segue mais facilmente, observando a regularidade de $G$ com $A$ e $f$ conforme a 3.3.9.

\subsection{Lipschitz continuidade com os dados}

Teorema 5.4.1 Chame $X_{\gamma}=\left(X_{0}, X_{1}\right)_{\gamma, \infty ; K}^{0}$ para $\gamma \in[0,1)$. Sejam $\alpha, \beta \in$ $[0,1), \alpha>\beta$. Seja $U \subset \mathbb{R} \times X_{\beta}$ um aberto. Suponha $(t, x) \rightarrow f(t, x): U \rightarrow$ $X_{0} e(t, x) \rightarrow A(t, x): U \rightarrow \mathcal{H}\left(X_{1}, X_{0}\right)$ Hölder continuas com $t$ e Lipschitz continuas com $x$.

Para um $\left(\tau_{0}, \xi\right) \in U$, seja $x(\cdot, \xi)$ a função que obedece de $d x / d t+A(t, x) x=$ $f(t, x), x\left(\tau_{0}\right)=\xi$ conforme teorema 5.3.1.

Seja $\left(\tau_{0}, \xi_{0}\right) \in U$. Então existe uma vizinhança $V \subset U \cap\left(\mathbb{R} \times X_{\alpha}\right)$ de $\left(\tau_{0}, \xi_{0}\right)$ e existe $T_{0}>0$ tal que toda $x(\cdot, \xi)$ existe em um intervalo $\left[\tau_{0}, \tau_{0}+T_{0}\right]$ quando $\left(\tau_{0}, \xi\right) \in V e$

$$
\|x(t, \xi+h)-x(t, \xi)\|_{X_{\beta}} \leq c(\alpha, \beta)\|h\|_{X_{\beta}},
$$

se $t \in\left[\tau_{0}, \tau_{0}+T\right] e\left(\tau_{0}, \xi\right),\left(\tau_{0}, \xi+h\right) \in V$.

Prova. Denotando $A(x(\xi))(s)=A(s, x(s, \xi))$, pela 4.0.16,

$$
\begin{aligned}
& x(t, \xi+h)-x(t, \xi)=U_{A(x(\xi))}(t, \tau) h \\
& +\int_{\tau}^{t} U_{A(x(\xi))}(t, s)(A(s, x(s, \xi))-A(x(s, \xi+h), s)) x(s, \xi+h) d s+ \\
& \int_{\tau}^{t} U_{A(x(\xi))}(t, s)(f(s, x(s, \xi+h))-f(s, x(s, \xi))) d s .
\end{aligned}
$$

A prova pode ser concluída tomando a norma em $X_{\beta}$, usando as estimativas de 4.0.12 e aplicando a desigualdade de Gronwall[[8],lema 7.1.2, página 189]. Note que o integrando da primeira integral tem ordem $O\left((t-s)^{-\beta}(s-\right.$ $\tau)^{\alpha-1}$ ) e obedece a exigência do lema, pois $1+\alpha-\beta>1$. 


\subsection{Diferenciabilidade e Analiticidade com os dados}

Teorema 5.5.1 Suponha as hipóteses do teorema 5.3.1. Suponha também que para cada $(\tau, \xi, \lambda, \mu)$ existe uma vizinhança no tempo $I=[\tau, \tau+T]$ com $T=T(\tau, \xi, \lambda, \mu)$ onde

$$
(u, \lambda) \rightarrow f(t, u, \lambda), f(t, u, \lambda) \in X_{0}
$$

$e$

$$
(u, \mu) \rightarrow A(t, u, \mu), A(t, u, \mu) \in L\left(X_{1}, X_{0}\right)
$$

tem derivadas até ordem $k, 1 \leq r \leq \infty$ uniformemente Hölder contínua com $t$ em $I$ e localmente Hölder ou Lipschitz continuas com $u, \lambda$ e com $u, \mu$. Então $U \times \Lambda \times \Upsilon \ni(\xi, \lambda, \mu) \rightarrow u(\cdot ; \tau, \xi, \lambda, \mu) \in C\left([\tau, \tau+T], X_{\alpha}\right)$ é uma função $C^{k}$. Temos analiticidade se as funções $f(t, u, \lambda)$ e $A(t, u, \mu)$ são analíticas uniformemente em I, isto é, suas séries de Taylor convergem uniformemente em $I$.

Prova. Para uma vizinhança de $\left(\tau_{0}, \xi_{0}, \mu_{0}, \lambda_{0}\right)$ a função $G$ é contração em um vizinhança de cada $w \in C\left([0, T], X_{\alpha}\right) \cap C^{\eta}\left([0, T], X_{\beta}\right)$. A função $w \rightarrow A(w): C\left([0, T], X_{\alpha}\right) \cap C^{\eta}\left([0, T], X_{\beta}\right) \rightarrow C^{\mu^{-}}\left([0, T], L\left(X_{1}, X_{0}\right)\right)$ é $C^{k}$ ou analítica pelas hipóteses e pelo 5.2 .1 para um $\mu^{-}$suficientemente pequeno. Raciocínio análogo para $f$ e aplicação de 3.3 .9 concluem a prova.

Observação 5.5.2 Amann obtém um teorema de diferenciabilidade com técnicas bem distintas em [[4]]. Usa equação singulares integrais do tipo de Volterra. Ambos os teoremas requerem melhor análise.

Teorema 5.5.3 Suponha além das hipóteses do teorema 5.6.1 que $(t, u, \lambda) \rightarrow$ $f(t, u, \lambda): U \times \Lambda \rightarrow X_{0} e(t, u, \mu) \rightarrow A(t, u, \mu): U \times \Upsilon \rightarrow L\left(X_{1}, X_{0}\right)$ é $C^{k}$ ou analítica. Então

$$
(t, \tau, \xi, \lambda, \mu) \rightarrow u(t ; \tau, \xi, \lambda, \mu)
$$

é $C^{k}$ ou analítica respectivamente para $t>\tau$ no domínio de existência.

Prova. Segue o mesmo argumento da prova de [Henry[8], página 66, corolário 3.4.6]. Seja $t=s / m+\tau$ para qualquer $m>0$. E também $y(s)=$ $y(s ; 0, \xi, \hat{\lambda}, \hat{\mu})$ definido por $y(s)=x(s / m+\tau, \tau, \xi, \lambda, \mu) \operatorname{com} \hat{\lambda}=(\lambda, m, \tau) \mathrm{e}$ $\hat{\mu}=(\mu, m, \tau)$. Então 
e

$$
\frac{d y}{d s}(s)=\frac{1}{m} \frac{d x}{d t}(s / m+\tau)
$$

$d y / d s+(1 / m) A(\tau+s / m, y, \mu) y=(1 / m) f(\tau+s / m, y, \lambda), y(0)=\xi$.

$\mathrm{O}$ resto do argumento é igual . Pela unicidade, se $t>\tau$ e para $m=$ $1 /(t-\tau)$

$$
x(t, \tau, \xi, \lambda, \mu)=y\left(1 ; 0, \xi,\left(\lambda,(t-\tau)^{-1}, \tau\right),\left(\mu,(t-\tau)^{-1}, \tau\right)\right)
$$

Aplicação do teorema 5.5.1 implica para $s>0$

$$
(\xi,(\lambda, m, \tau),(\mu, m, \tau) \rightarrow y(s ; o, \xi,(\lambda, m, \tau),(\mu, m, \tau))
$$

é $C^{r}$ ou analítica. E o resultado está provado.

\subsection{Exemplo}

O objetivo desta seção é demonstrar como os resultados do presente capítulo e anteriores podem ser usados para obter solução para o seguinte sistema em $\theta$ e $c$

$$
\begin{aligned}
& \theta_{t}=\operatorname{div}(\mathrm{K}(\lambda, \theta) \nabla \theta)+\mathrm{R}(\lambda, \theta, \mathrm{c}) \\
& c_{t}=\operatorname{div}(\mathrm{D}(\lambda, \theta) \nabla \mathrm{c})+\mathrm{S}(\lambda, \theta, \mathrm{c}), \mathrm{t}>0, \mathrm{x} \in \Omega \\
& \frac{\partial \theta}{\partial N}=P(\lambda, \theta), \frac{\partial c}{\partial N}=Q(\lambda, \theta, c), t>0, x \in \partial \Omega
\end{aligned}
$$

$\operatorname{com} \theta(0, x)=\theta_{0}(x)$ e $c(0, x)=c_{0}(x)$.

\subsubsection{Existência}

\section{Hipóteses}

1. Todas as funções são $C^{\infty}$ em relação à variáveis .

2. O sistema é parabólico: $K(\lambda, r)>0$ e $D(\lambda, r)>0$ para $\lambda, r \in \mathbb{R}$.

3. O domínio $\Omega \in \mathbb{R}^{n}$ tem fronteira regular e existe uma função $c_{\delta}, \delta>0$, que obedece $\left|c_{\delta}(x)\right|<\delta$, se $x \in \Omega$, e também $c_{\delta}(x)=0, \partial c_{\delta} / \partial N(x)=1$ se $x \in \partial \Omega$. A função $c_{\delta}$ pode ser estendita e, então, definida em $\mathbb{R}^{n}$. 
Em princípio, o teorema de existência e unicidade para equação de evolução parabólica não é aplicável. Segundo o método usual, a condição de fronteira é incorparada ao domínio do operador associado ao problema. Mas então seu domínio torna-se, em geral, não constante sem constituir um espaço linear, condições necessárias para a aplicação do teorema.

A técnica usada para tornar o sistema tratável é a mudança de variável. Não é minha intenção incluir todos os passos para executar a mudança. Mais detalhes na minha dissertação[20].

\section{Funções para mudança}

$\operatorname{Sejam}(\lambda, x, r) \rightarrow \Psi^{1}(\lambda, x, r): \mathbb{R} \times \mathbb{R}^{n} \times \mathbb{R} \rightarrow \mathbb{R},(\lambda, x, r, s) \rightarrow \Psi^{2}(\lambda, x, r, s):$ $\mathbb{R} \times \mathbb{R}^{n} \times \mathbb{R}^{2}$ descritas por

$$
\Psi^{1}(\lambda, x, r)=r+P(\lambda, r) c_{\delta}(x), \Psi^{2}(\lambda, x, r, s)=s+Q(\lambda, r, s) c_{\delta}(x) .
$$

Suponha $l>0$. É possível achar um número $\delta>0$ para

$$
\begin{gathered}
\left|\Psi^{1}(\lambda, x, r)-r\right| \leq 1,\left|\Psi^{2}(\lambda, x, r, s)-s\right| \leq 1, \\
\left|\Psi_{r}^{1}-1\right| \leq 1 / 2,\left|\Psi_{s}^{2}-1\right| \leq 1 / 2,
\end{gathered}
$$

se $|\lambda| \leq 1, x \in \Omega$ e $|r|,|s| \leq l+1$.

Seja então $v^{1}$ e $v^{2}$ as funções que vão substituir $\theta$ e $c$ no sistema definidas por

$$
\theta(t, x)=\Psi^{1}\left(\lambda, x, v^{1}(t, x)\right), c(t, x)=\Psi^{2}\left(\lambda, x, v^{1}(t, x), v^{2}(t, x)\right) .
$$

\section{Mudança de variáveis}

Após um cálculo comprido, mas constituído apenas de aplicações da regra da cadeia para derivadas, o sistema em relação à $v^{1}$ e $v^{2}$ torna-se

$$
\begin{aligned}
& v_{t}^{1}=K\left(\lambda, \Psi^{1}\right) \Delta v^{1}+\frac{1}{\Psi_{r}^{1}} F^{1}\left(\lambda, x, v^{1}, \nabla v^{1}\right)+\frac{1}{\Psi_{r}^{1}} R\left(\lambda, \Psi^{1}, \Psi^{2}\right) \\
& v_{t}^{2}=\frac{\Psi_{r}^{2}}{\Psi_{s}^{2}}\left(D\left(\lambda, \Psi^{1}\right)-K\left(\lambda, \Psi^{1}\right)\right) \Delta v^{1}+D\left(\lambda, \Psi^{1}\right) \Delta v^{2}-\frac{\Psi_{r}^{2}}{\Psi_{r}^{1} \Psi_{s}^{2}} F^{1}\left(\lambda, x, v^{1}, \nabla v^{1}\right) \\
& -\frac{\Psi_{r}^{2}}{\Psi_{r}^{1} \Psi_{s}^{2}} R\left(\Psi^{1}, \Psi^{2}\right)+\frac{1}{\Psi_{s}^{2}} F^{2}\left(\lambda, x, v^{1}, v^{2}, \nabla v^{1}, \nabla v^{2}\right)+\frac{1}{\Psi_{s}^{2}} S\left(\lambda, \Psi^{1}, \Psi^{2}\right), \quad t> \\
& \frac{\partial v^{1}}{\partial N}=\frac{\partial v^{2}}{\partial N}=0, \quad t>0, x \in \partial \Omega, \\
& \Psi^{1}\left(\lambda, x, v_{0}^{1}\right)=\theta_{0}, \Psi^{2}\left(\lambda, x, v_{0}^{1}, v_{0}^{2}\right)=c_{0}, \quad x \in \Omega .
\end{aligned}
$$




\section{Notação}

1. Os termos $F^{1}$ e $F^{2}$ denotam

$$
F^{1}\left(x, v^{1}, v^{2}\right)=K_{r}\left(\lambda, \Psi^{1}\right)\left|\nabla_{T} \Psi^{1}\right|^{2}+K\left(\lambda, \Psi^{1}\right)\left[\Psi_{r r}^{1}\left|\nabla v^{1}\right|^{2}+2 \nabla \Psi_{r}^{1} . \nabla v^{1}+\Delta \Psi^{1}\right]
$$

$\mathrm{e}$

$$
\begin{gathered}
F^{2}\left(\lambda, x, v^{1}, v^{2}, \nabla v^{1}, \nabla v^{2}\right) \\
=D_{r}\left(\lambda, \Psi^{1}\right)\left|\nabla_{T} \Psi^{2}\right|^{2}+D\left(\lambda, \Psi^{1}\right)\left[\Delta \Psi^{2}+\Psi_{r r}^{2}\left|\nabla v^{1}\right|^{2}+\Psi_{s s}^{2}\left|\nabla v^{2}\right|^{2}+\right. \\
\left.2\left(\nabla \Psi_{r}^{2} \cdot \nabla v^{1}+\nabla \Psi_{s}^{2} \cdot \nabla v^{2}+\Psi_{r s} \nabla v^{1} \cdot \nabla v^{2}\right)\right] .
\end{gathered}
$$

2. Está implícito $\Psi^{1}=\Psi^{1}\left(\lambda, x, v^{1}\right)$ e $\Psi^{2}=\Psi^{2}\left(\lambda, x, v^{1}, v^{2}\right)$.

3. O símbolo $\nabla_{T}$ significa gradiente total, isto é, $\nabla_{T} \Psi^{1}\left(\lambda, x, v^{1}\right)=\nabla \Psi^{1}+$ $\Psi_{r}^{1} \nabla v^{1}$, e $\nabla$ significa gradiente somente em relação a $x$. Também $\Delta$ siginifica Laplaciano em relação a $x$.

Antes da mudança, o sistema possui uma forma diagonal, mas mascarada. Com a mudança, surgem termos fora da diagonal.

\section{Teorema de existência e unicidade}

Com notação óbvia, o sistema pode ser descrito por

$$
\begin{aligned}
& v_{t}^{1}=a_{11}\left(\lambda, x, v^{1}\right) \Delta v^{1}+b_{1}\left(\lambda, x, v^{1}, v^{2}, \nabla v^{1}, \nabla v^{2}\right) \\
& v_{t}^{2}=a_{21}\left(\lambda, x, v^{1}\right) \Delta v^{1}+a_{22}\left(\lambda, x, v^{1}\right) \Delta v^{2}+b_{2}\left(\lambda, x, v^{1}, v^{2}, \nabla v^{1}, \nabla v^{2}\right), t>0, x \in \Omega \\
& \frac{\partial v^{1}}{\partial N}=\frac{\partial v^{2}}{\partial N}=0, \quad t>0, x \in \partial \Omega, \\
& \Psi^{1}\left(\lambda, x, v_{0}^{1}\right)=\theta_{0}, \Psi^{2}\left(\lambda, x, v_{0}^{1}, v_{0}^{2}\right)=c_{0}, \quad x \in \Omega .
\end{aligned}
$$

$\operatorname{com} a_{11}(\lambda, x, r), a_{21}(\lambda, x, r), a_{22}(\lambda, x, r), b_{1}(\lambda, x, r, s, p, q), b_{2}(\lambda, x, r, s, p, q)$ funções $C^{\infty}$ para $x \in \bar{\Omega},|r|,|s| \leq l+1, p, q \in \mathbb{R}^{n}$.

Defina o operador

$$
A(\lambda, v)=\left|\begin{array}{cc}
-a_{11}\left(\lambda, \cdot, v^{1}\right) \Delta & 0 \\
-a_{21}\left(\lambda, \cdot, v^{1}\right) \Delta & -a_{22}\left(\lambda, \cdot, v^{1}\right) \Delta
\end{array}\right|+k I
$$

com $k$ um número real positivo a escolher, $I$ o operador identidade e $v=$ $\left(v_{1}, v_{2}\right)^{t}, D(A(\lambda, v))=X_{1}=W_{N}^{2, p}(\Omega) \times W_{N}^{2, p}(\Omega)$. Aí, $W_{N}^{2, p}(\Omega)$ um subspaço 
fechado do espaço de Sobolev $W^{2, P}(\Omega)$ cujos elementos obedecem à condição de Neumann na fronteira.

Seja também

$$
f(\lambda, v)=\left(b_{1}-k, b_{2}-k\right)^{t} .
$$

Então o sistema pode ser descrito pela equação de evolução no espaço de Banach $X_{0}=L_{p}(\Omega) \times L_{p}(\Omega)$,

$$
v_{t}+A(\lambda, v) v=f(\lambda, v), t>0,
$$

e $v(0)(x)=\left(v_{0}^{1}(0, x), v_{0}^{2}(0, x)\right)^{t}$

Defina espaço de intermediária $X_{\alpha}=\left(X_{0}, X_{1}\right)_{\alpha, \infty ; K}^{o}$. Conforme observação 1.2.31, a imersão $X_{\alpha} \subset C^{\nu}(\Omega) \times C^{\nu}(\Omega)$ ocorre quando $0 \leq \nu<2 \alpha-n / p$. Seja $0<\beta<\alpha<1, \beta>1 / 2$ e seja $p>n$ para que $\nu>1$.

Defina por $V$ o aberto em $X_{\beta}$

$$
V=\left\{v \in X_{\beta},\|v\|_{C(\bar{\Omega}) \times C(\bar{\Omega})}<l\right\}
$$

Portanto as funções $(\lambda, v) \rightarrow A(\lambda, v):[-1,1] \times V \rightarrow L\left(X_{1}, X_{0}\right)$ e $(\lambda, v) \rightarrow$ $f(\lambda, v):[-1,1] \times V \rightarrow X_{0}$ estão bem definidas e são $C^{\infty}$.

A regularidade segue, por exemplo, da aplicação do Recíproco do terema de Taylor[[8], página 12] com a observação de que tem-se as imersões $X_{\beta} \subset$ $C^{1} \times C^{1}$ e $C^{0} \times C^{0} \subset X_{0}$.

Conforme demonstrado em minha dissertação[[20], página 41], com uma escolha conveniente de $k$, o operador $A(\lambda, v)$ é setorial, isto é, obedece 1 de 1.2.13, para cada $\lambda \in[-1,1], v \in V$. Pela condição de parabolicidade, $D(A(\lambda, v)) \doteq X_{1}$ uniformemente em $V$. Então $A(\lambda, v) \in \mathcal{H}\left(X_{1}, X_{0}\right)$ se $\lambda \in$ $[-1,1]$ e $v \in V$.

Então para um $T>0$, o teorema de existência e unicidade se aplica e obtemos solução regular em $C\left([0, T), X_{\alpha}\right) \cap C^{1}\left((0, T), X_{0}\right)$ se $v_{0} \in X_{\alpha}$. A derivada $d v / d t \in X_{\gamma}$ é Hölder contínua em relação à $t>0$ se $\gamma \in(0, \alpha-\beta)$. Como $v(\tau) \in X_{1}, \tau>0$, a $d v / d t(t) \in X_{\gamma}$, para $\gamma \in(0,1-\beta), t>\tau$. Então também supondo $p>n$ para $2(1-\beta)-n / p>0$, as derivadas estão em $C^{\delta}((0, T) \times \Omega)$, para um $\delta>0$. Também a função $\left(\lambda, v_{0}\right) \rightarrow v\left(\cdot, \lambda, v_{0}\right)$ : $[-1,1] \times V \rightarrow C\left([0, T], X_{\alpha}\right)$ é regular.

Para cada $t>0$, e $v$ a solução obtida, o problema elíptico em $u, A(\lambda, v) u=$ $f(\lambda, v)-v_{t}$, com todas funções $C^{\delta}(\Omega)$ com $x$, então toda solução $u$ aí é $C^{2+\delta}(\Omega)$. Em particular também $v$ é e obtemos uma solução clássica para o sistema. 


\subsubsection{Bifurcação de Hopf}

Sob certas condições, é possível que ocorra bifucação de Hopf em relação ao parâmetro $\lambda$ para o mesmo sistema

$$
\begin{aligned}
& \theta_{t}=\operatorname{div}(\mathrm{K}(\lambda, \theta) \nabla \theta)+\mathrm{R}(\lambda, \theta, \mathrm{c}) \\
& c_{t}=\operatorname{div}(\mathrm{D}(\lambda, \theta) \nabla \mathrm{c})+\mathrm{S}(\lambda, \theta, \mathrm{c}), \mathrm{t}>0, \mathrm{x} \in \Omega \\
& \frac{\partial \theta}{\partial N}=P(\lambda, \theta), \frac{\partial c}{\partial N}=Q(\lambda, \theta, c), t>0, x \in \partial \Omega
\end{aligned}
$$

Além das hipóteses da seção 5.6.1, suponha também

1. Se $\lambda \in(-1,1), R(\lambda, 0,0)=S(\lambda, 0,0)=P(\lambda, 0)=Q(\lambda, 0,0)=P_{r}(\lambda, 0)=$ $Q_{r}(\lambda, 0,0)=Q_{s}(\lambda, 0,0)=0$.

2. Para $\lambda \in(-1,1), K(\lambda, 0)=D(\lambda, 0)$.

3. $R_{r}(0,1,0)+R_{s}(0,0,0)-R_{s}(0,0,1)+S_{s}(0,0,1)=0$.

4.Para $H$ definido a seguir, suponha $\operatorname{det}\left(\partial_{1} H(0,0)\right)>0$.

5. $\left(R_{r}(\lambda, 1,0)+R_{s}(\lambda, 0,0)-R_{s}(\lambda, 0,1)+S_{s}(\lambda, 0,1)\right)^{2}-4 \operatorname{det}\left(\partial_{1} H(0, \lambda)\right)<0$ se $\lambda \in(-1,1)$.

6. $\partial_{\lambda}\left[R_{r}(\lambda, 1,0)+R_{s}(\lambda, 0,0)-R_{s}(\lambda, 0,1)+S_{s}(\lambda, 0,1)\right]_{\lambda=0} \neq 0 \mathrm{e}$

$$
\frac{K_{\lambda}(0,0)}{\partial_{\lambda}\left[R_{r}(\lambda, 1,0)+R_{s}(\lambda, 0,0)-R_{s}(\lambda, 0,1)+S_{s}(\lambda, 0,1)\right]_{\lambda=0}} \leq 0 .
$$

Sob estas restrições, o sistema possui uma bifurcação de Hopf em $\lambda=0$. Especificamente, para todo $\alpha \in(0,1)$, existe um número $h_{0}>0$ e funçoes regulares $\lambda:\left(-h_{0}, h_{0}\right) \rightarrow \mathbb{R}, \rho:\left(-h_{0}, h_{0}\right) \rightarrow \mathbb{R}$ e $u:\left(-h_{0}, h_{0}\right) \rightarrow$ $C^{\alpha+1}\left(\mathbb{R}, X_{0}\right) \cap C^{\alpha}\left(\mathbb{R}, X_{1}\right)$ tais que

$$
\lambda(0)=0, \rho(0)=0, u(c) \text { é não constante se } h \neq 0,
$$

e $u(h)$ é uma solução periódica com período $2 \pi \rho(h) / \omega$ do sistema com $u(h)=$ $(\theta(h), c(h))^{t}$ e $t \in \mathbb{R}$.

Prova.

Linearização 
É conveniente denotar

$$
A(v, \lambda)=\left[\begin{array}{cc}
-K\left(\lambda, \Psi^{1}\right) & 0 \\
-\frac{\Psi_{r}^{2}}{\Psi_{s}^{2}}\left[\left(D\left(\lambda, \Psi^{1}\right)-K\left(\lambda, \Psi^{1}\right)\right]\right. & -D\left(\lambda, \Psi^{1}\right)
\end{array}\right] .
$$

e chamando de $H(v, \lambda)$ a parte remanescente, o sistema torna-se

$$
v_{t}=\Phi(v, \lambda)
$$

$\Phi(v, \lambda)=-A(v, \lambda) v+H(v, \lambda), v \in X_{1}$.

A linearização em $(0, \lambda)$ será $\partial_{1} \Phi(0, \lambda)=-A(0, \lambda)+\partial_{1} H(0, \lambda)$ e $\partial_{1} H(0, \lambda)$

$$
=\left[\begin{array}{cc}
R_{r}(\lambda, 1,0)+R_{s}(\lambda, 0,0) & R_{s}(\lambda, 0,1) \\
-R_{r}(\lambda, 1,0)-R_{s}(\lambda, 0,0)+S_{r}(\lambda, 1,0)+S_{s}(\lambda, 0,0) & -R_{s}(\lambda, 0,1)+S_{s}(\lambda, 0,1)
\end{array}\right] .
$$

Então $\operatorname{det}\left(\partial_{1} H(0, \lambda)\right)=S_{s}(\lambda, 0,1)\left[R_{r}(\lambda, 1,0)+R_{s}(\lambda, 0,0)\right]-R_{s}(\lambda, 0,1)\left[S_{r}(\lambda, 1,0)+\right.$ $\left.S_{s}(\lambda, 0,0)\right]$.

A função $X_{1} \times(-1,1) \rightarrow X_{0}:(x, \lambda) \rightarrow \Phi(x, \lambda)$ está bem definida e é $C^{\infty}$. A conclusão da prova segue da aplicação conjunta de 5.6.2 e 5.6.3.

Observação 5.6.1 Existem funções $R, S, K, D$ que obedecem 1 a 6 . Suponha, para cada $\lambda \in(-1,1)$,

$$
R_{r}(\lambda, 1,0)+R_{s}(\lambda, 0,0)=\lambda,
$$

e

$$
-R_{s}(\lambda, 0,1)+S_{s}(\lambda, 0,1)=\lambda, S_{s}(\lambda, 0,1) \neq \lambda
$$

e $K_{\lambda}(0,0)<0$.

As condições 2,3 e 6 estão automaticamente satisfeitas e $\partial_{1} H(0, \lambda)=$ $\lambda S_{s}(\lambda, 0,1)+\left(\lambda-S_{s}(\lambda, 0,1)\right)\left(S_{r}(\lambda, 1,0)+S_{s}(\lambda, 0,0)\right)$. Admita $\partial H_{1}(0, \lambda)=1$. Obrigatoriamente

$$
S_{r}(\lambda, 1,0)+S_{s}(\lambda, 0,0)=\frac{1-\lambda S_{s}(\lambda, 0,1)}{\lambda-S_{s}(\lambda, 0,1)} .
$$

Portanto também valem 4,5. Especificamente, se $R=\lambda r^{2} / 2+s^{2}, S_{s}(\lambda, 0,1)=$ $\lambda+2$, então $S_{r}(\lambda, 1,0)+S_{s}(\lambda, 0,0)=\left(\lambda^{2}+2 \lambda-1\right) / 2$. Uma escolha possivel é $S(\lambda, r, s)=(\lambda+2) s^{2} / 2+\left(\lambda^{2}+2 \lambda-1\right) r / 2$. O obediência de 1 também está garantida. 


\section{Espectro de um sistema linear elíptico}

Lema 5.6.2 Seja $\lambda \in(-1,1)$. Nosso objetivo é delimitar o espectro do problema elíptico em $L_{q}(\Omega) \times L_{q}(\Omega)$ para $q \in(1, \infty)$

$$
\begin{array}{cc}
{\left[\begin{array}{cc}
a(\lambda) & 0 \\
0 & a(\lambda)
\end{array}\right]\left[\begin{array}{c}
\Delta u^{1} \\
\Delta u^{2}
\end{array}\right]+\left[\begin{array}{ll}
b_{11}(\lambda) & b_{12}(\lambda) \\
b_{21}(\lambda) & b_{22}(\lambda)
\end{array}\right]\left[\begin{array}{l}
u^{1} \\
u^{2}
\end{array}\right]=\mu(\lambda)\left[\begin{array}{c}
u^{1} \\
u^{2}
\end{array}\right], x \in \Omega,} \\
\frac{\partial u^{1}}{\partial N}=\frac{\partial u^{2}}{\partial N}=0, x \in \partial \Omega . & (*)_{\lambda}
\end{array}
$$

Admita as condições

$$
\begin{aligned}
& \text { 1. }\left|\begin{array}{ll}
b_{11}(0) & b_{12}(0) \\
b_{21}(0) & b_{22}(0)
\end{array}\right|>0 . \\
& \text { 2. } b_{11}(0)+b_{22}(0)=0 . \\
& \text { 3. } b_{11}(\lambda)+b_{22}(\lambda)-4\left|\begin{array}{ll}
b_{11}(\lambda) & b_{12}(\lambda) \\
b_{21}(\lambda) & b_{22}(\lambda)
\end{array}\right|<0 . \\
& \text { 4. } b_{11}^{\prime}(0)+b_{22}^{\prime}(0) \neq 0 \text { e } \frac{a^{\prime}(0)}{b_{11}^{\prime}(0)+b_{22}^{\prime}(0)}<0 .
\end{aligned}
$$

\section{Consequências}

1. $\left\{ \pm \omega_{0} i\right\}, \omega_{0}=\left|\begin{array}{ll}b_{11}(0) & b_{12}(0) \\ b_{21}(0) & b_{22}(0)\end{array}\right|$, são autovalores simples de $(*)_{0}$.

2. (*) não tem autovalores da forma $\left\{k \omega_{0} i\right\}, k \neq \pm 1$.

3. $\partial_{\lambda} \Re \mu(0) \neq 0$.

Prova. O argumento é uma adaptação de [[6],página 58].

Denote por $0=\nu_{0}>\nu_{1}>\cdots>\nu_{i}>\cdots$ os autovalores do Laplaciano com condição de Neumann e $\left\{\phi_{i}\right\}_{i \geq 0}$ o sistema de autofunções completo em $L^{2}(\Omega), \Delta \phi_{i}=\nu_{i} \phi_{i}$ em $\Omega$ e $\partial \phi_{i} / \partial N=0$ em $\partial \Omega$. O espectro e as autofunções se mantém caso o problema seja resolvido em $L_{q}(\Omega), q \in(1, \infty)[[6]$,página 58]. O domínio $\Omega$ é um conjunto regular e então as autofunções também o são.

Se $u^{1}, u^{2}$ obedecem ao problema $(*)_{\lambda}$ em $L_{q}(\Omega) \times L_{q}(\Omega)$ então $u^{1}=$ $\sum_{i=0}^{\infty} p_{i} \phi_{i}$ e $u^{2}=\sum_{i=0}^{\infty} q_{i} \phi_{i}$. Também $\Delta u^{1}=\sum_{i=0}^{\infty} p_{i} \nu_{i} \phi_{i}$ e $\Delta u^{2}=\sum_{i=0}^{\infty} q_{i} \nu_{i} \phi_{i}$. $\mathrm{O}$ problema $(*)_{\lambda}$ é equivalente ao conjunto de equações para $i \geq 0$,

$$
\left[\begin{array}{cc}
a(\lambda) \nu_{i}+b_{11}(\lambda) & b_{12}(\lambda) \\
b_{21}(\lambda) & a(\lambda) \nu_{i}+b_{22}(\lambda)
\end{array}\right]\left[\begin{array}{c}
p_{i} \\
q_{i}
\end{array}\right]=\mu(\lambda)\left[\begin{array}{c}
p_{i} \\
q_{i}
\end{array}\right]
$$


Portanto, o espectro de $(*)_{\lambda}$ é a união de todos os espectros de

$$
\left[\begin{array}{cc}
a(\lambda) \nu_{i}+b_{11}(\lambda) & b_{12}(\lambda) \\
b_{21}(\lambda) & a(\lambda) \nu_{i}+b_{22}(\lambda)
\end{array}\right]
$$

para $i \geq 0$. Sua equação característica tem soluções

$$
\mu(\lambda)=\frac{2 a(\lambda) \nu_{i}+b_{11}(\lambda)+b_{22}(\lambda) \pm \sqrt{\left(b_{11}(\lambda)+b_{22}(\lambda)\right)^{2}-4\left|\begin{array}{ll}
b_{11}(\lambda) & b_{12}(\lambda) \\
b_{21}(\lambda) & b_{22}(\lambda)
\end{array}\right|}}{2} .
$$

Sob as condições do enunciado seguem então as consequências descritas.

Teorema da bifurcação de Hopf para equações totalmente nãolineares em espaços de Banach de Lunardi e Da Prato

Teorema 5.6.3 Seponha $X_{0}, X_{1}$ espaços de Banach. Seja $(x, \lambda) \rightarrow F(x, \lambda)$ : $U \times(-1,1) \rightarrow X_{0}, U$ um conjunto aberto de $X_{1}$, uma funçẫo de classe $C^{2}$ com derivadas $\partial^{k} F / \partial x^{k}$ Lipschitz contínuas com relação à $x, k \geq 2$. Assuma $A(\lambda)=F_{x}(0, \lambda)$ um operator setorial para $\lambda=0$ e \pm wi autovalores simples isolados da sua complexificação $\bar{A}(\lambda)$ e kwi, $k \neq \pm 1, k$ inteiro, contidos no seu conjunto resolvente. Suponha também a condição de trasversalidade

$$
\partial_{\lambda}(\mathbb{R e} \bar{A}(\lambda))_{\lambda=0} \neq 0
$$

Então para todo $\alpha \in(0,1)$, existe $c_{0}>0$ e funções $C^{k-1}, \lambda:\left(-c_{0}, c_{0}\right) \rightarrow$ $\mathbb{R}, \rho:\left(-c_{0}, c_{0}\right) \rightarrow \mathbb{R}, u:\left(-c_{0}, c_{0}\right) \rightarrow C^{\alpha+1}\left(\mathbb{R}, X_{0}\right) \cup C^{\alpha}\left(\mathbb{R}, X_{1}\right)$, tais que

$$
\lambda(0)=0, \rho(0)=0, u(c) \text { é não constante para } c \neq 0,
$$

e u(c) é uma solução periódica com período $2 \pi \rho(c) / \omega$ de

$$
u^{\prime}=F(u, \lambda(c)), t \in \mathbb{R} .
$$

Prova. Enunciado com mais detalhes e demonstração em [[15], página 381, teorema 9.3.3] . Referência original [14]. 


\section{Referências Bibliográficas}

[1] P.E.Sobolevskii, Equations of parabolic type in a Banach space, Amer. Math. Soc. Translation (2), 49(1965), 1-62.

[2] H. Tanabe, Equations of Evolution, Pitman, London, 1979.

[3] P. L .Butzer e H. Berens, Semi-Groups of Operators and Approximation, Springer-Verlag, Berlin, 1967.

[4] H. Amann, Dynamic Theory of Quasilinear Parabolic Equations - I. Abstract Evolution Equations, Nonlinear Analysis, 12(1988), 895-919.

[5] H. Amann, Linear and Quasilinear Parabolic Problems, Volume I, Abstract Linear Theory, Monographs in Mathematics, Birkhäuser, Berlin, 1995.

[6] H.Amann, Hopf Bifurcation in Quasilinear Reaction-Diffusion Systems, , Delay Differential Equations and Dynamical System, Proceedings. Lec. Notes in Math. 14551455, Springer-Verlag, Berlin (1991).

[7] F.Hoppenstadt, Asymptotic series solutions of some nonlinear parabolic equations with a small parameter, Arch. Rat. Mech. Anal., 35(1969), 284-298.

[8] D.B.Henry, Geometric Theory of Semilinear Parabolic Equations, Lectures Notes in Mathematics, Springer-Verlag, Berlin, 1981.

[9] N. Dunford e J.T. Schwartz, Linear Operators. Part I: General Theory. Interscience, New York, 1957.

[10] Tosio Kato, Abstract evolution equation of parabolic type in Banach and Hilbert spaces, Nagoya Math. J., 5(1961), 93-125. 
[11] A.Pazy, Semigroups of linear operators and applications to partial differential equations, Springer-Verlag, New Yourk, 1983.

[12] S.N.Chow e J.K.Hale, Methods of Bifurcation Theory, Springer-Verlag, New York, 1982.

[13] D.B.Henry, Banach spaces calculs, notas de aula para o curso Cálculo em espaço de Banach, USP, Brasil, 2000.

[14] Alessandra Lunardi, Giuseppe Da Prato, Hopf bifurcation for fully nonlinear equations in Banach space, Ann. Ins. Henry Poincaré, 3(1986), 315-329.

[15] Alessandra Lunardi, Analytic Semigroups and Optimal Regularty in Parabolic Problems, Birkhäuser, Boston, 1995.

[16] Alessandra Lunardi, Analyticity of the maximal solution of an abstract nonlinear parabolic equation, Nonlinear Analysis, 6(1982), 503-521.

[17] G. Da Prato, P. Grisvard, Equation d'evolutions abstraites non linéares de type parabolique, Ann. Mat. Pura Appl., 120(1979), 329-396.

[18] G.Prodi e A.Ambrosetti, Analisi non lineare, Pubbl. Classe Scienze Scu. norm. sup., Pisa, 1973.

[19] E. Sinestrari, On the Abstract Problem of Parabolic Type in Spaces of Continuos Functions, Journal of Mathematical Analysis and Applications, 107(1985), 16-66.

[20] A.S.Munhoz, Estudo de um sistema de reação difusão com condições de fronteira não-lineares, dissertação de mestrado, IME-USP, 1998. (pedido de cópia pelo e-mail munhoz-br@yahoo.com.br). 